University of Tennessee Health Science Center

UTHSC Digital Commons

\title{
Molecular Mechanisms Underlying Alcohol-Induced Cerebral Artery Smooth Muscle BK Channel Inhibition and Eventual Cerebral Vasoconstriction
}

Guruprasad Kuntamallappanavar

University of Tennessee Health Science Center

Follow this and additional works at: https://dc.uthsc.edu/dissertations

Part of the Medical Molecular Biology Commons

\section{Recommended Citation}

Kuntamallappanavar, Guruprasad, "Molecular Mechanisms Underlying Alcohol-Induced Cerebral Artery Smooth Muscle BK Channel Inhibition and Eventual Cerebral Vasoconstriction" (2015). Theses and Dissertations (ETD). Paper 371. http://dx.doi.org/10.21007/etd.cghs.2015.0169. 


\title{
Molecular Mechanisms Underlying Alcohol-Induced Cerebral Artery Smooth Muscle BK Channel Inhibition and Eventual Cerebral Vasoconstriction
}

\begin{abstract}
Introduction and Rationale: Ethanol $(\mathrm{EtOH})$ at concentrations obtained in circulation during moderate to heavy episodic drinking, such as during binge drinking (30-60 $\mathrm{mM})$ causes cerebral vasoconstriction in many species, including humans. Using rodents as a model to study ethanolinduced cerebral artery constriction, our laboratory demonstrated that ethanol-induced cerebral artery constriction is due to druginduced reduction of STOCs (Spontaneous Transient Outward Currents) in cerebral artery smooth muscle. In this tissue, STOCs result from the activity of large conductance, calcium-and voltage-gated potassium (BK) channels. Indeed, ethanol (50 mM) decreases the steady-state activity (NPo) of vascular myocyte BK channels leading to an increase in cerebral artery tone. In native tissues, functional BK channels are oligomers of four channel-forming slo1 subunits that are associated with small, accessory subunits $(\beta 1-4)$. $\beta$ subunits do not form channels themselves but modify BK current phenotype, including its pharmacology. In particular, the vascular smooth muscle-abundant BK $\beta 1$ subunit is required for ethanol to inhibit cerebral artery myocyte BK channels under physiological conditions of voltage and calcium. In contrast, the neuronally-predominant $\beta 4$ subunit does not support this ethanol action. The molecular bases of ethanol-mediated inhibition of $\beta 1$ subunit-containing BK channels and resulting cerebral vasoconstriction remain unknown.
\end{abstract}

Objective: Identify the BK $\beta 1$ subunit regions and $\beta 1$ subunit-dependent channel gating mechanisms underlying ethanol-induced inhibition of cerebral artery smooth muscle BK channel inhibition and eventual cerebral artery constriction.

Methods: Combination of recombinant DNA and other molecular biology in vitro approaches, patch-clamp electrophysiology, allosteric gating modeling, reversible permeabilization of arteries with cDNAs, and artery pressurization techniques.

Results: Ethanol sensitivity of slo1 current is dependent on the channel's activating ion, i.e., $\mathrm{Ca} 2+\mathrm{i}$. Moreover, ethanol-induced modification of activity of slo1 (cbv1) and heteromeric cbv $1+\beta 1$ BK channels is primarily due to modulation of calcium-driven gating: in particular, increase in $\mathrm{Ca} 2+\mathrm{i}$ affinity; decrease in allosteric interaction between 1) RCK and voltage sensing domains and 2) RCKs and pore gate domains. Ethanol facilitation of channel inhibition is favored by $\beta 1$-and $\beta 2$ but not $\beta 3$ or $\beta 4$ subunits. Consistent with the involvement of calciumdependent mechanism, the former two drastically increase the channel's apparent calcium sensitivity whereas the latter fail to do so. Transmembrane domains of BK- $\beta 1$ subunit are essential for ethanol-mediated inhibition of $\beta 1$-containing BK channels. In particular, the second transmembrane domain of $\beta 1$ subunit is necessary for both inhibition of $\beta 1$-containing $B K$ channels and cerebral artery constriction evoked by ethanol.

Conclusion: BK $\beta 1$ subunit TM2 enables ethanol-induced inhibition of $\beta 1$-containing BK channels and cerebral artery constriction, with drug action on channel activity being dependent on modification of calcium-gating parameters.

\section{Document Type}

Dissertation

\section{Degree Name}

Doctor of Philosophy (PhD) 


\section{Program}

Biomedical Sciences

Research Advisor

Alejandro M. Dopico, Ph.D.

\section{Keywords}

Alcohol/Ethanol, BK Channel/Maxi K Channel, Cerebrovascular Diseases, Electrophysiology, Vascular Smooth Muscle Cell, Vasoconstriction

\section{Subject Categories}

Medical Molecular Biology | Medical Sciences | Medicine and Health Sciences 


\title{
Molecular Mechanisms Underlying Alcohol-Induced Cerebral Artery Smooth Muscle BK Channel Inhibition and Eventual Cerebral Vasoconstriction
}

\author{
A Dissertation \\ Presented for \\ The Graduate Studies Council \\ The University of Tennessee \\ Health Science Center \\ In Partial Fulfillment \\ Of the Requirements for the Degree \\ Doctor of Philosophy \\ From The University of Tennessee
}

By

Guruprasad Kuntamallappanavar

May 2015 
Copyright $(\subset) 2015$ by Guruprasad Kuntamallappanavar. All rights reserved. 


\section{DEDICATION}

I dedicate this dissertation to

my father Chandrappa K.M., to my mother Channabasamma K.M. and to my brothers Kotresh K.M. and Gururaj K.M.

for their unconditional love and support. 


\section{ACKNOWLEDGEMENTS}

I would like express my special appreciation and thanks to my dissertation advisor Dr. Alejandro M. Dopico, who always made me to understand the principles of science, hard work and diligence. His scientific acumen, selfless devotion to profession and research, motivation, patience, intellectual guidance, and unreserved fatherhood help led to enrich the soul of the author beyond this dissertation and is what carried me through many-a-difficult time during my research. I deeply thank him for offering many opportunities during my Ph.D. training. He is the one who taught me how to think scientifically and present professionally my research to the scientific community. I am extremely grateful to him for giving me an opportunity to pursue graduate education under his supervision and for instilling in me the qualities of a good scientific investigator. My sincere wish is to collaborate with him in future and keep learning a lot.

I also deeply thank my dissertation advisory committee members Drs.Charles W. Leffler, Kafait U. Malik, William E. Armstrong and Steven J. Tavalin for their valuable suggestions, guidance, and scientific criticism throughout my project which enabled me to complete my dissertation work. It has been a privilege to count with this lineup of superb scientist as dissertation committee members.

I am using this opportunity to express my gratitude to Dr.Anna N.Bukiya, who supported me throughout graduate studies in Dr. Dopico's lab. I am thankful for her aspiring guidance, invaluably constructive criticism, brilliant comments and suggestions, and friendly advice during my dissertation project. Without her supervision and constant help this dissertation would not have been possible.

I extend my gratitude to all other members of Dr. Dopico's laboratory, Drs. Aditya K. Singh, Yanping Ye, Man Zhang and Shivakumar Bangalore, and to Mrs. Maria T. Asuncion-Chin for their pleasant co-operation, constructive criticism, timely help and suggestions throughout my dissertation work without which I would not be able complete this project in a timely fashion.

I would like to thank the American Heart Association for awarding me a predoctoral fellowship. I also like to thank the Biophysical Society, the Research Society on Alcoholism and the College of Graduate Health Sciences for awarding me educational travel awards, which facilitated the presentation of my work in scientific meetings.

I would like show my appreciation to Mr. Larry Tague, Ms. Shirley Hancock and all other members of the ETD advisory committee for their kind advice and help in formatting this dissertation and make it presentable to the scientific world. Thanks to all my friends and colleagues in Memphis for their love and support over the years.

I am highly grateful to the Integrated Biomedical Science Program, the College of Graduate Health Sciences and the University of Tennessee Health Science Center for 
providing me a wonderful opportunity to pursue my doctoral education. I am also grateful to the pharmacology department administrative staff for their help and cooperation.

I have reached this milestone today because of the unconditional love, patience and support of my father, Chandrappa K.M, my mother, Channabasamma K.M., and brothers, Kotresh K.M. and Gururaj K.M., whose sacrifices I can never repay. They served as a great inspiration in each and every step of my life, suggested me to take life one step at a time, and never to give up even in the face of overwhelming odds. I am deeply thankful to my best friend Deepthi Gururaj, who stayed by me through thick and thin in life. Finally, I genuinely would like to thank God for granting me the capability to journey successfully in life. 


\begin{abstract}
Introduction and Rationale: Ethanol $(\mathrm{EtOH})$ at concentrations obtained in circulation during moderate to heavy episodic drinking, such as during binge drinking (30-60 mM) causes cerebral vasoconstriction in many species, including humans. Using rodents as a model to study ethanol-induced cerebral artery constriction, our laboratory demonstrated that ethanol-induced cerebral artery constriction is due to drug-induced reduction of STOCs (Spontaneous Transient Outward Currents) in cerebral artery smooth muscle. In this tissue, STOCs result from the activity of large conductance, calcium-and voltagegated potassium $(\mathrm{BK})$ channels. Indeed, ethanol $(50 \mathrm{mM})$ decreases the steady-state activity $\left(\mathrm{NP}_{\mathrm{o}}\right)$ of vascular myocyte $\mathrm{BK}$ channels leading to an increase in cerebral artery tone. In native tissues, functional BK channels are oligomers of four channel-forming slo1 subunits that are associated with small, accessory subunits ( $\beta 1-4)$. $\beta$ subunits do not form channels themselves but modify BK current phenotype, including its pharmacology. In particular, the vascular smooth muscle-abundant BK $\beta 1$ subunit is required for ethanol to inhibit cerebral artery myocyte BK channels under physiological conditions of voltage and calcium. In contrast, the neuronally-predominant $\beta 4$ subunit does not support this ethanol action. The molecular bases of ethanol-mediated inhibition of $\beta 1$ subunitcontaining BK channels and resulting cerebral vasoconstriction remain unknown.
\end{abstract}

Objective: Identify the BK $\beta 1$ subunit regions and $\beta 1$ subunit-dependent channel gating mechanisms underlying ethanol-induced inhibition of cerebral artery smooth muscle BK channel inhibition and eventual cerebral artery constriction.

Methods: Combination of recombinant DNA and other molecular biology in vitro approaches, patch-clamp electrophysiology, allosteric gating modeling, reversible permeabilization of arteries with cDNAs, and artery pressurization techniques.

Results: Ethanol sensitivity of slo1 current is dependent on the channel's activating ion, i.e., $\mathrm{Ca}^{2+}$ i. Moreover, ethanol-induced modification of activity of slo1 (cbv1) and heteromeric cbv $1+\beta 1 \mathrm{BK}$ channels is primarily due to modulation of calcium-driven gating: in particular, increase in $\mathrm{Ca}^{2+}{ }_{i}$ affinity; decrease in allosteric interaction between 1) RCK and voltage sensing domains and 2) RCKs and pore gate domains. Ethanol facilitation of channel inhibition is favored by $\beta 1$-and $\beta 2$ but not $\beta 3$ or $\beta 4$ subunits. Consistent with the involvement of calcium-dependent mechanism, the former two drastically increase the channel's apparent calcium sensitivity whereas the latter fail to do so. Transmembrane domains of BK- $\beta 1$ subunit are essential for ethanol-mediated inhibition of $\beta 1$-containing BK channels. In particular, the second transmembrane domain of $\beta 1$ subunit is necessary for both inhibition of $\beta 1$-containing BK channels and cerebral artery constriction evoked by ethanol.

Conclusion: BK $\beta 1$ subunit TM2 enables ethanol-induced inhibition of $\beta 1$-containing BK channels and cerebral artery constriction, with drug action on channel activity being dependent on modification of calcium-gating parameters. 


\section{TABLE OF CONTENTS}

\section{CHAPTER 1. INTRODUCTION ....................................................................................1}

1.1. Cerebrovascular Diseases and Binge Drinking .........................................................

1.1.1. Epidemiology of Cerebrovascular Diseases ..........................................

1.1.2. Alcohol Consumption and Health ............................................................

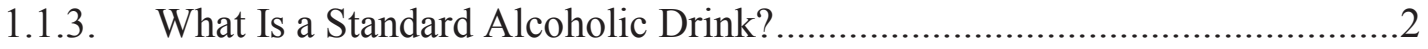

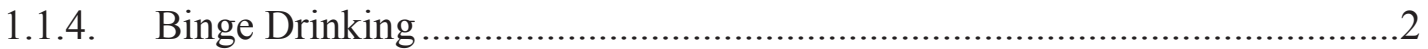

1.1.5. Binge Drinking as a Risk Factor for Cerebrovascular Diseases ...................2

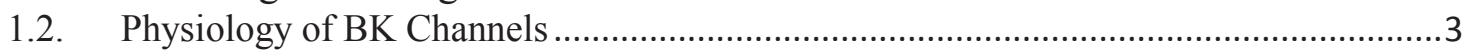

1.2.1. Physiological Role of BK Channels in Arterial Smooth Muscle ..................3

1.2.2. Basic Structure of BK Channels....................................................................4

1.2.3. Beta Subunits of BK Channels ..................................................................

1.2.4. Role of BK $\beta 1$ Subunit in Regulation of Arterial Tone .............................. 7

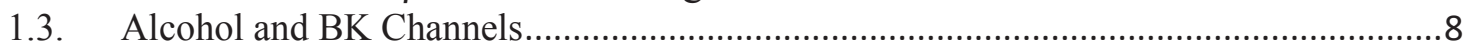

1.3.1. Ethanol Modulation of BK Channel Activity ............................................

1.3.2. Factors Modulating Ethanol Action of BK Channel Activity ......................8

1.3.3. Role of BK $\beta 1$ Subunit in Ethanol-Induced Cerebral Vasoconstriction .......9

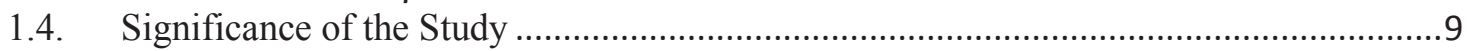

CHAPTER 2. HYPOTHESIS ..................................................................................

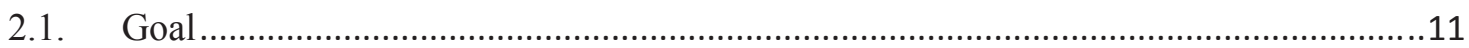

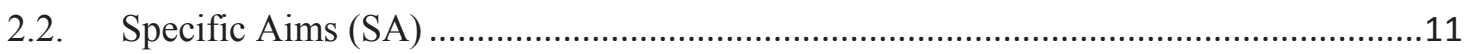

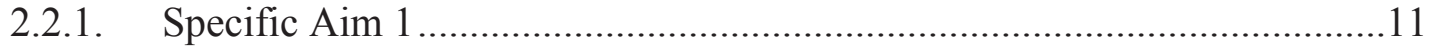

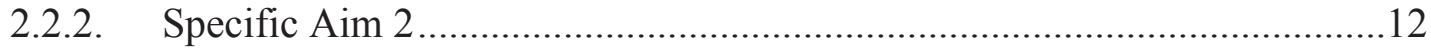

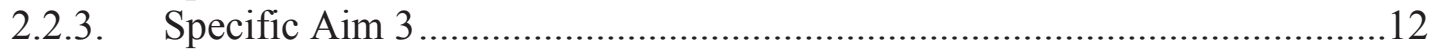

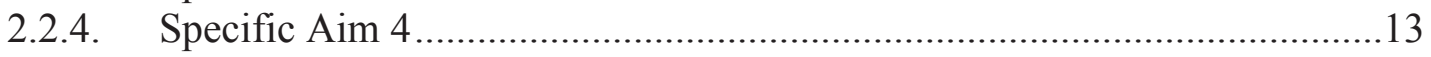

CHAPTER 3. MATERIALS AND METHODS..........................................................14

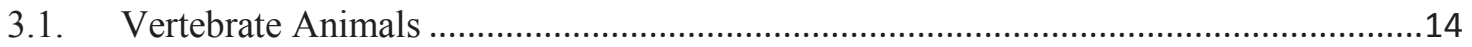

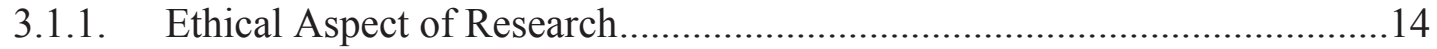

3.1.2. Information of Veterinary Care of Animals ............................................ 14

3.1.3. Procedures to Minimize Discomfort, Distress, Pain and Injury...................14

3.1.4. Method of Euthanasia.......................................................................... 15

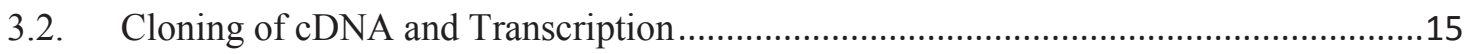

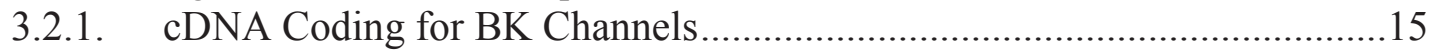

3.2.2. cDNA Coding for BK Channel Accessory Beta Subunits ..........................16

3.3 Oocyte Isolation from Xenopus Leavis and RNA Injection ........................................16

3.3.1. Model Justification: Xenopus Leavis Oocytes..........................................16

3.3.2. Oocyte Isolation from Xenopus Leavis Oocytes ......................................17

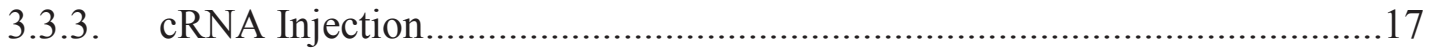

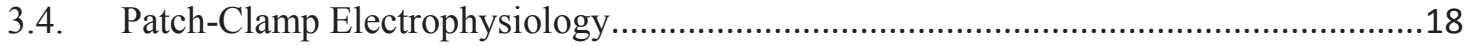

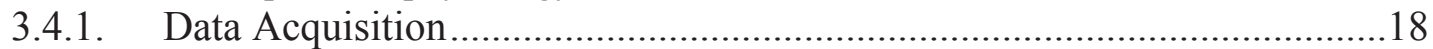

3.4.2. Estimating the Number of BK Channels in the Excised Patch ...................20

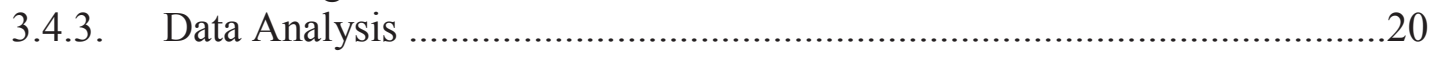




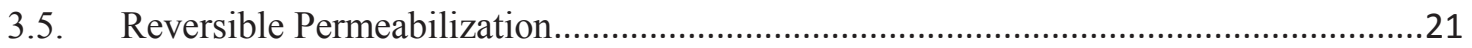

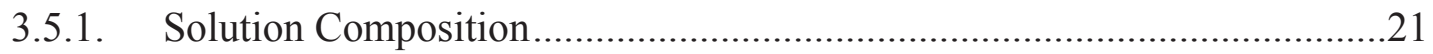

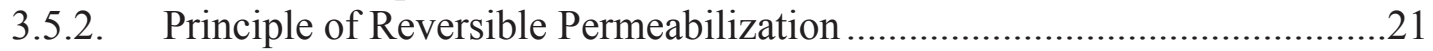

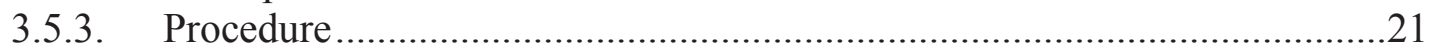

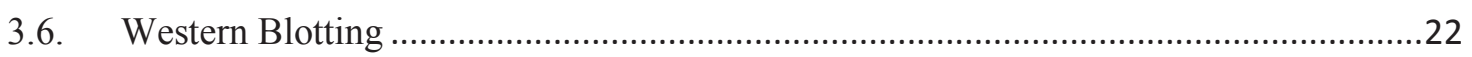

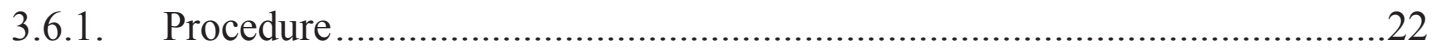

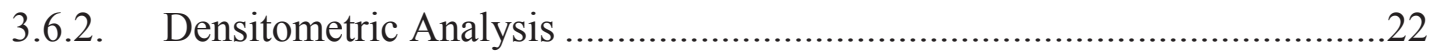

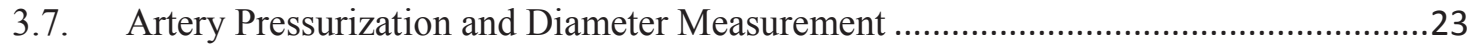

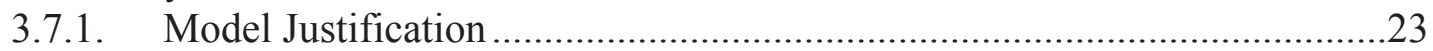

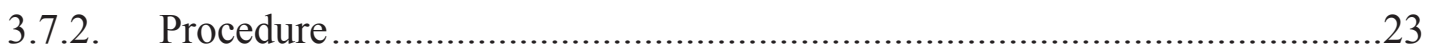

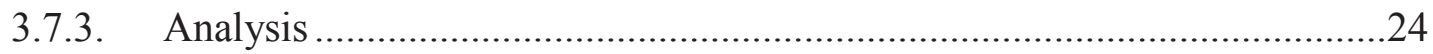

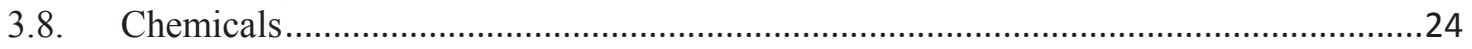

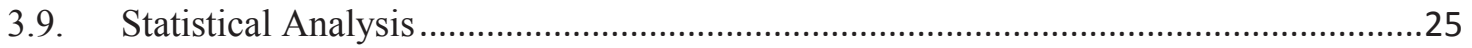

\section{CHAPTER 4. THE CHANNEL'S ACTIVATING ION DETERMINES ETHANOL MODULATION OF BK CHANNELS ...................................................26}

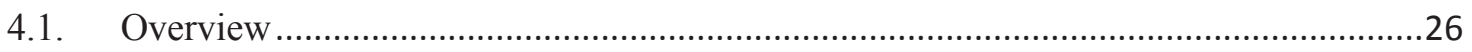

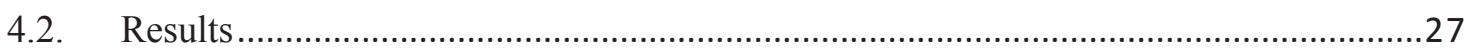

4.2.1. Ethanol Modulation of Slo1 Channel's Activity Is Selectively

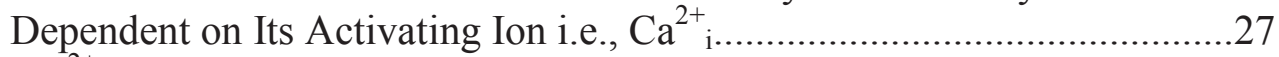

4.2.2. $\mathrm{Mg}^{2+}$-/Voltage-Gated Cbv1 Channels Are Ethanol Insensitive ..................27

4.2.3. Slo2 Channels Are Ethanol Insensitive over a Wide Range of Their Activating Ion Concentration .................................................................. 31

4.2.4. Slo3 Channels Are Ethanol Insensitive over a Wide Range of Their Activating Ion Concentration .......................................................................33

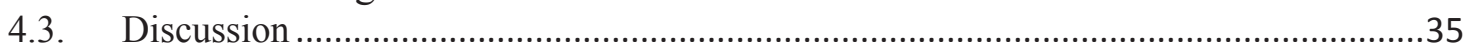

\section{CHAPTER 5. ETHANOL DISTINCTLY MODULATES BETA1 AND BETA2-} CONTAINING BK CHANNELS ....................................................................................38

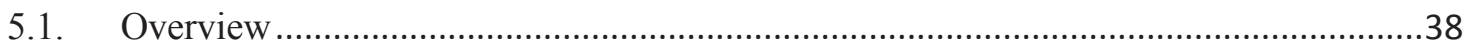

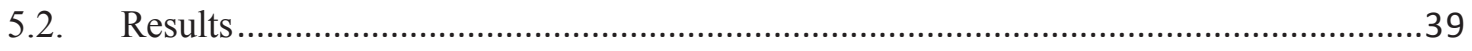

5.2.1. Auxillary Beta Subunits Functionally Co-Express with Channel Forming Cbv1 Subunits........................................................................39

5.2.2. $\beta 1$ Subunit Facilitates Ethanol-Induced Inhibition of $\beta 1$-Containing BK Channels .....................................................................................4 41

5.2.3. Ethanol Modulation of $w t \beta 2$ - and $\beta 2$-IR-Containing BK Channel Activity Is Similar to the Alcohol Response of $\beta 1$ Subunit-Containing BK Channels.

5.2.4. Ethanol Modulation of $\beta 3$ - and $\beta 4$-Containing BK Channel Activity Is Similar to the Alcohol Response of Homomeric Cbv1 Channels ...............47

5.2.5. Understanding BK Channel Function Requires a Complex Allosteric Model.

5.2.6. Determining Ethanol's Effect on the Movement of Voltage Sensors for Homomeric BK Channels

5.2.7. Determining Ethanol Effect on the Closed-to-Open Conformational Change on Homomeric Cbv1 Channels 
5.2.8. Determining Ethanol's Effect on the Remaining Voltage-Dependent

Parameters of Cbv1 Channel Gating

5.2.9. Determining Ethanol Effect on Homomeric BK Channel's $\mathrm{Ca}^{2+}{ }_{\mathrm{i}}$ Binding Affinity

5.2.10. Determining Ethanol Effect on BK Channel Voltage Sensor Movement in Presence of $\beta 1$ Subunits

5.2.11. Determining Ethanol Effect on the Closed-to-Open Conformational Change and Voltage-Dependent Parameters in $\beta 1$-Containing BK Channels

5.2.12. Determining Ethanol Effect on the $\mathrm{Ca}^{2+}{ }_{\mathrm{i}}$ Binding Affinity of $\beta 1$ Containing BK Channels

5.3. Discussion

\section{CHAPTER 6. SPECIFIC BETA1 REGION(S) IS NECESSARY FOR ETHANOL-INDUCED INHIBITION OF BK CHANNELS AND EVENTUAL CEREBRAL ARTERY CONSTRICTION .................................................................70}

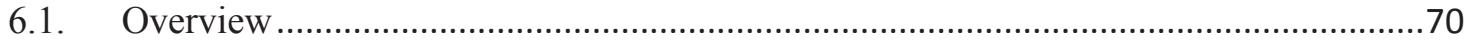

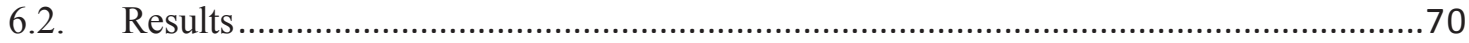

6.2.1. $\quad \beta 1 / \beta 4$ Chimeric Constructs and Their Functional Characterization .............70

6.2.2. Both Transmembrane Domains of BK- $\beta 1$ Subunits Are Responsible for Ethanol-Induced Inhibition of BK Channels...............................................71

6.2.3. Second Transmembrane Domain of BK- $\beta 1$ Subunit Determines the Ethanol-Induced Inhibition of BK Channels

6.2.4. Reversible Permeabilization Leads to Overexpression of Desired Proteins and Resulting Functional Middle Cerebral Arteries

6.2.5. Ethanol-Induced Cerebral Artery Constriction Is Determined by $\beta 1$ Subunit of BK Channels .............................................................................76

6.2.6. BK $\beta 1$ Subunit Is Second Transmembrane Domain Is Necessary for Ethanol-Induced Cerebral Artery Constriction ............................................79

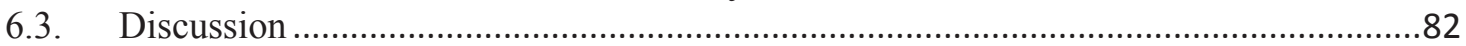

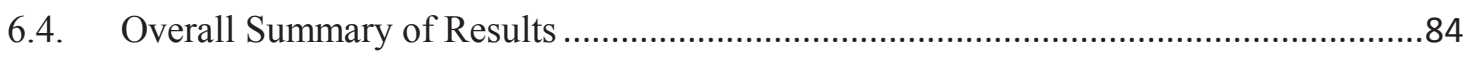

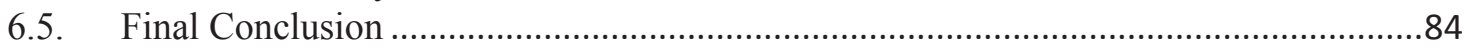

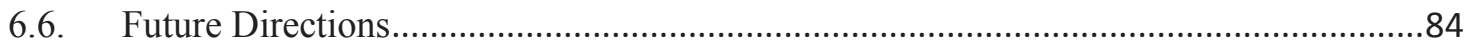

LIST OF REFERENCES .....................................................................................................85

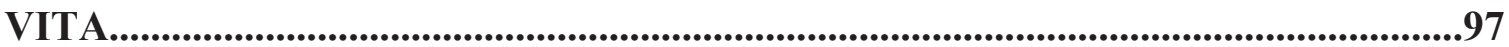




\section{LIST OF TABLES}

Table 4-1. Summary of key structural features of different slo channel family members, their activating ligands and sensitivity to $50 \mathrm{mM}$ ethanol

Table 5-1. Summary of various allosteric model parameters and their likely corresponding structural BK channel domains

Table 5-2. Summary of best fit parameters obtained for homomeric BK channel in presence or absence of $50 \mathrm{mM}$ ethanol ....................................................64

Table 5-3. Summary of best fit parameters obtained for $\beta 1$-contianing BK channel in presence or absence of $50 \mathrm{mM}$ ethanol..... 


\section{LIST OF FIGURES}

Figure 1-1. Role of BK channels in regulation of vascular tone …….............................5

Figure 1-2. Schematic structure of BK channels containing accessory subunit $(\beta 1-4) \ldots . .66$

Figure 4-1. Ethanol effect on BK channel activity is $\mathrm{Ca}^{2+}{ }_{i}$-dependent.............................28

Figure 4-2. Ethanol action on BK channel activity is function of $\mathrm{Ca}^{2+}{ }_{i}$.........................29

Figure 4-3. $\mathrm{Mg}^{2+}$ /voltage-gated cbv1 channels are ethanol-insensitive ..........................30

Figure 4-4. Slo2.1:slo2.2 channels are insensitive to ethanol at a wide range of activating $\mathrm{Na}^{+}{ }_{i}$

Figure 4-5. Slo3 channels are ethanol-insensitive at a wide range of activating $\mathrm{OH}^{-}$......34

Figure 5-1. Macroscopic currents after cbv1+ $\beta 1-4$ subunit expression in Xenopus laevis oocytes show characteristic features of BK currents

Figure 5-2. Ethanol activates $\beta 1$-containing BK channels at submicromolar $(0.3 \mu \mathrm{M})$

$\mathrm{Ca}^{2+}{ }_{\mathrm{i}}$, while causing strong inhibition at higher $(100 \mu \mathrm{M}) \mathrm{Ca}^{2+}{ }_{\mathrm{i}}$.

Figure 5-3. BK $\beta 1$ subunits determine ethanol-induced inhibition of BK channels at physiological $\mathrm{Ca}^{2+}{ }_{\mathrm{i}}(3-45 \mu \mathrm{M})$ and voltages $(-60$ to $-20 \mathrm{mV})$

Figure 5-4. Ethanol-mediated inhibition of $w t \beta 2$-containing BK channels is similar to the ethanol response of cbv1 $+\beta 1$ channels.

Figure 5-5. Ethanol-mediated inhibition of $\beta 2$-IR-containing BK channels is similar to the ethanol response of cbv1 $+\beta 1$ channels

Figure 5-6. Ethanol responses of $\beta 3$ - or $\beta 4$-containing BK currents mimic those of homomeric BK (cbv1) channels.

Figure 5-7. Allosteric model for activation of BK channel

Figure 5-8. Ethanol modulation of voltage dependence of homomeric BK (cbv1) channel activation kinetics in absence of $\mathrm{Ca}^{2+}{ }_{i}$

Figure 5-9. Ethanol does not alter the parameters associated with closed-to-open conformational change and voltage dependence of cbv1 channels. 55

Figure 5-10. Ethanol does not alter the voltage-dependent parameters of cbv1 channels

Figure 5-11. Ethanol modulates the parameters associated with $\mathrm{Ca}^{2+}{ }_{\mathrm{i}}$ binding affinity

of cbv1 channels 
Figure 5-12. Ethanol modulation of voltage-dependent activation kinetics from $\beta 1$ containing BK channels in the absence of $\mathrm{Ca}^{2+}{ }_{\mathrm{i}}$

Figure 5-13. Ethanol does not alter the voltage-dependent parameters of cbv1+ $\beta 1$ channels

Figure 5-14. Ethanol modulates the parameters associated with $\mathrm{Ca}^{2+}{ }_{\mathrm{i}}$ binding affinity of cbv $1+\beta 1$ channels.

Figure 6-1. Cartoons of $\beta 1 / \beta 4$ chimeras constructed for this study.

Figure 6-2. BK $\beta 1$ transmembrane domains determine ethanol-mediated inhibition of $\beta 1$-containing BK channel

Figure 6-3. Second transmembrane domain of BK $\beta 1$ subunit determines the ethanol-induced inhibition of $\beta 1$-containing BK channel

Figure 6-4. Reversible permeabilization leads to overexpression BK $\beta 1$ or $\beta 1 / \beta 4$ chimeric protein and functioning mouse middle cerebral artery

Figure 6-5. BK $\beta 1$ subunit determines ethanol-induced constriction of middle cerebral artery.

Figure 6-6. Second transmembrane domain of BK $\beta 1$ subunit determines ethanolinduced constriction of middle cerebral artery.....

Figure 6-7. Second transmembrane domain of BK $\beta 1$ subunit is responsible for ethanol-induced constriction of middle cerebral artery 


\section{LIST OF ABBREVIATIONS}

$\beta 2-I R$

ATP

BAC

BK

$\mathrm{Ca}^{2+}{ }_{\mathrm{i}}$

$\left[\mathrm{Ca}^{2+}\right]_{\mathrm{i}}$

$\mathrm{CaCl}_{2}$

$\mathrm{Cl}^{-}$

CTD

Ctx

DMEM

EC

EDHF

EDRF

EGTA

$\mathrm{EtOH}$

$\mathrm{G}$

$\mathrm{G} / \mathrm{G}_{\max }$

HA

HEDTA

HEPES

Ibtx

IC

$\mathrm{I} / \mathrm{O}$

$\mathrm{KCl}$

KCNMB 1

$\mathrm{K} / \mathrm{O}$

$\mathrm{KOH}$

$\mathrm{K}_{\mathrm{V}}$

LCA

$\mathrm{Mg}^{2+}$

$\left[\mathrm{Mg}^{2+}\right]$

$\mathrm{MgCl}_{2}$

MOPS

MS-222

MWC

$\mathrm{N}$

$\mathrm{Na}^{2+}$

$\left[\mathrm{Na}^{2+}\right]$

$\mathrm{NaCl}$

$\mathrm{NP}_{\mathrm{o}}$

NPS

$\mathrm{OH}^{-}$
Beta2 subunit without N-terminus inactivation peptide

Adenosine 5'-triphosphate

Blood alcohol concentration

Big (large) conductance, calcium- and voltage-gated potassium

Calcium

Intracellular calcium concentration

Calcium chloride

Chloride

Cytosolic tail domain

Charybdotoxin

Dulbecco's modified eagle medium

Extracellular side

Endothelium derived hyperpolarizing factor

Endothelium derived repolarizing factor

Ethylene glycol-bis(2-aminoethylether)-N,N,N',N'-tetraacetic acid

Ethanol

Macroscopic channel conductance

Ratio of conductance to maximum conductance

Horrigan Aldrich

$\mathrm{N}-\{2-[$ Bis(carboxymethyl)amino]ethyl $\}-\mathrm{N}-(2$-ydroxyethyl)glycine

2-[4-(2-hydroxyethyl)piperazin-1-yl]ethanesulfonic acid

Iberotoxin

Intracellular side

Inside-out

Potassium chloride

Gene encoding for BK- $\beta 1$ protein

Knock-out

Potassium hydroxide

Voltage-gated potassium channels

Lithocholate

Magnesium

Intracellular $\mathrm{Mg}^{2+}$ concentration

Magnesium chloride

3-propainesulfonic acid

Tricaine methanesulfonate

Monad-Wayman-Changuex

Number of channel in the membrane patch

Sodium

Sodium ion concentration

Sodium chloride

Index of channel steady-state

Nitroprusside

Hydroxyl ion 
PBS

PGD

$\mathrm{P}_{\mathrm{o}}$

PSS

RCK

STOC

TBS

TBS-T

TES

TM

Ryr

$\mathrm{V}_{0.5}$

VDCC

$\mathrm{V}_{\max }$
Phosphate buffer saline

Pore gate domain

Channel open probability

Physiological saline solution

Regulator of conductance for potassium

Spontaneous transient outward current

Tris-buffered solution

TBS with $0.1 \%$ tween

Tetraetyhyl Silane

Transmembrane

Ryanodine receptor

Voltage required to achieve half-maximal conductance

Voltage dependent calcium channels

Voltage required to maximal conductance 


\section{CHAPTER 1. INTRODUCTION}

\subsection{Cerebrovascular Diseases and Binge Drinking}

\subsubsection{Epidemiology of Cerebrovascular Diseases}

Cerebrovascular disease is a leading cause of long-term disability in the United States (U.S.) and other western countries and ranks No.4 among all causes of death (www.cdc.gov). In particular, stroke ranks third among all causes of mortality. On average, every four minutes someone dies of stroke. In 2007, stroke accounted for 1 in 18 deaths in the U.S. Moreover, the total expenditure on stroke in the U.S. is estimated to be \$43 billion per year (www.cdc.gov; Beaglehole., 1990; Mohler et al., 1999; Callahan., 2001) (Beaglehole 1990; Mohler et al. 1999; Callahan 2001).

\subsubsection{Alcohol Consumption and Health}

Alcohol is one the most commonly abused drugs. Excessive alcohol consumption is considered to be the $3^{\text {rd }}$ life-style related cause of death in the U.S., leading to 88,000 deaths annually (www.cdc.gov; www.niaaa.nih.gov).

Alcohol drinking pattern can be arbitrarily classified into the three following categories:

\subsubsection{Light Drinking}

Drinking less than 2 drinks per day, usually leading to Blood Alcohol Concentration (BAC) of 0.002-0.005\% (1-20 mM).

\subsubsection{Moderate Drinking}

Drinking less than 4 drinks per day, which usually leads to BAC of $0.15-0.25 \%$ (35-50 mM).

\subsubsection{Heavy drinking}

Drinking more than 4 drinks per day, usually leading to BAC of $0.25-0.50 \%$ (50$>100 \mathrm{mM}$ ). 


\subsubsection{What Is a Standard Alcoholic Drink?}

In the United States, a standard alcoholic "drink" refers to 14 grams ( 0.6 ounce or 1.2 table spoons) of pure alcohol (ethyl alcohol, ethanol, EtOH) (www.niaaa.nih.gov). This much amount of pure alcohol is usually found in

- 12 ounces of beer, which contains $5 \%$ of alcohol

- 5-ounces of wine, which typically contains $12 \%$ of alcohol

- 1.5 ounces of $80 \%$ distilled spirits or liquor (e.g., gin, rum, whiskey, vodka), which on average contain $40 \%$ alcohol.

\subsubsection{Binge Drinking}

National Institute of Alcohol Abuse and Alcoholism (NIAAA) defines binge drinking as moderate-to-heavy episodic drinking, in which a man consumes 5 or more drinks or an adult woman consumes 4 or more drinks in about 2 hours. (www.niaaa.gov).

Unfortunately, binge drinking remains a major health problem in the U.S, representing the major form of excessive alcohol abuse: approximately $92 \%$ of U.S. adults who drink excessively report binge drinking in the past 30 days; more than 38 million adults binge drink every year; on average 2 in 3 high schoolers who drink, do so by binge drinking. Overall, binge drinking causes more than 88,000 deaths in the U.S. every year (www.cdc.gov). Binge drinking has been shown to be associated with short term and long term major health problems, including cardiovascular and cerebrovascular diseases. (Wechsler et al. 2002; Barnett et al. 2003).

\subsubsection{Binge Drinking as a Risk Factor for Cerebrovascular Diseases}

Epidemiological studies show that moderate to heavy episodic drinking, such as binge drinking, significantly increases the risk for cerebrovascular spasm, brain hemorrhage and stroke (Klatsky et al. 1986; Moore and Pearson 1986; Marmot and Brunner 1991; Puddey et al. 1999; Gil-Nunez and Villanueva 2001). Binge drinking with blood alcohol concentration (BAC) of 0.15-0.50\% (35-80 $\mathrm{mM})$ is associated independently of any other factor with an increased risk for stroke and deaths from ischemic stroke (Haapaniemi et al. 1997; Zakhari 1997; Suter and Vetter 1999; Reynolds et al. 2003). Cerebrovascular disease associated with moderate-heavy episodic alcohol consumption is independent of beverage type and alcohol metabolism but linked to ethanol itself (Arkwright et al. 1982; Altura et al. 1983; Altura and Altura 1984; Zakhari 1997). Moderate to heavy drinking causes cerebral artery constriction, which has been related to cerebral vasospasm, ischemia, and stroke (Arkwright et al. 1982; Rimm et al. 1996; Wannamethee and Shaper 1996).

Direct ethanol exposure of different vasculatures may result in vasodilation or vasoconstriction depending on species, ethanol concentration, and type of vascular bed 
(Altura et al. 1983; Altura and Altura 1984; Potter and Beevers 1984; Jackson et al. 1985; Puddey et al. 1985; Puddey et al. 1987). However, there is strong evidence that acute exposure to ethanol $>18 \mathrm{mM}$ produces constriction of cerebral arteries, including in humans. Constriction of cerebral arteries is due to ethanol itself, and replicated in vivo and in vitro models used to study ethanol-induced cerebral artery constriction in several species (Altura and Altura 1982; Altura et al. 1983; Altura and Altura 1984; Puddey et al. 1987; Marmot and Brunner 1991; Zhang et al. 1993; Gordon et al. 1995; Seppa et al. 1996; Wannamethee and Shaper 1996; Liu et al. 2004).

Rat and mouse arteries have proved successful in vivo and in vitro models to study ethanol-induced cerebral artery constriction and stroke (Altura and Altura 1982; Altura et al. 1983; Altura and Altura 1984; Zhang et al. 1993; Gordon et al. 1995; Liu et al. 2004; Bukiya et al. 2009; Bukiya et al. 2011). However, the mechanisms underlying ethanol-induced cerebrovascular constriction remained largely unknown for many years. Several studies have suggested that endogenously produced vasoactive compounds, such as endothelial factors (EDRF, EDHF) or neural factors from the vascular smooth musclesurrounding parenchyma modulate ethanol-induced vasoconstriction (Jackson et al. 1985; Iwase et al. 1995). However, ethanol-induced constriction of cerebral arteries is evoked by ethanol acting primarily on the arterial smooth muscle itself (Altura and Altura 1982; Altura et al. 1983; Altura and Altura 1984; Zhang et al. 1993; Gordon et al. 1995; Yang et al. 2001; Liu et al. 2004). In a major breakthrough towards identifying the molecular targets mediating ethanol action on cerebral arteries, our group discovered that ethanol at concentrations obtained in circulation during moderate-to-heavy binge drinking (10-100 $\mathrm{mM}$ ) constricted rat and mouse cerebral arteries via inhibition of BK channel activity in cerebral arterial smooth muscle (Liu et al. 2004; Bukiya et al. 2009; Bukiya et al. 2011).

\subsection{Physiology of BK Channels}

\subsubsection{Physiological Role of BK Channels in Arterial Smooth Muscle}

Resistant size cerebral arteries are main determinants of controlling blood flow, whether systemically or locally. Under physiological conditions, resistant size arteries exist in a partially constricted state, from which they may dilate or further constrict depending on the perfusion needs and metabolic demands of tissues and organs. Intravascular pressure is considered one of the major stimuli for such arterial constriction. Arterial myocytes present some degree of contraction in response to intravascular pressure, which is known as myogenic tone as reported in cerebral arteries (Jaggar and Nelson 2000; Jaggar et al. 2000; Wellman et al. 2002; Wellman and Nelson 2003). Indeed, myocytes respond to increase in intravascular pressure by graded membrane depolarization, which leads to $\mathrm{Ca}^{2+}$ influx via depolarization-activated Voltage-dependent $\mathrm{Ca}^{2+}$ Channels (VDCC). The resulting increase in global cytosolic $\mathrm{Ca}^{2+}{ }_{\mathrm{i}}$ leads to smooth muscle contraction and thus cerebral artery constriction. On the other hand, BK channels participate in a negative-feedback loop that limits the depolarization-triggered by increase in $\mathrm{Ca}^{2+}$, myogenic tone and vasoconstriction, as found in many species and vessels 
including rabbit (Brayden and Nelson 1992), and rat cerebral (Erdos et al. 2002), and human pial arteries (Wellman et al. 2002; Wellman and Nelson 2003). This key role of $\mathrm{BK}$ channel is due to its unique position as transducer between cell metabolism and membrane potential: a rise in internal $\mathrm{Ca}^{2+}$ leads to $\mathrm{BK}$ channel activation, which generates positive outward currents that drive the myocyte membrane potential in a negative direction and, eventually, close voltage-dependent $\mathrm{Ca}^{2+}$ channels. Thus, BK channel activation counteracts contraction due to voltage-dependent $\mathrm{Ca}^{2+}$ influx, causing vascular smooth muscle relaxation and eventual vasodilation. Conversely, a decrease in BK activity blunts the negative feedback on voltage-dependent $\mathrm{Ca}^{2+}$ influx, leading to vasoconstriction (Brayden and Nelson 1992; Asano et al. 1993; Jaggar and Nelson 2000; Jaggar et al. 2000; Cheng and Lederer 2008; Subramani et al. 2009) (Figure 1-1).

\subsubsection{Basic Structure of BK Channels}

BK channels are members of the slo channel family, first identified in Drosophila melanogaster. The gene encoding for BK channel is called Slo or Slol or KCNMA1. In mammalian tissues, BK channels formed from tetrameric association of channel-forming $\alpha$ subunits. There are three slo channel family members, namely slo1, slo2 and slo3. The protein products of all Slo channel family members share basic very distinct characteristic features (Xia et al. 2004; Salkoff et al. 2006), such as 1. High selectivity for $\mathrm{K}^{+}$ions 2. Large unitary conductance and 3. Activation by intracellular Ligands (For example, Slo1 by $\mathrm{Ca}^{2+}$; $\mathrm{Slo} 2$ by $\mathrm{Na}^{2+}$; $\mathrm{Slo} 3$ by $\mathrm{OH}^{-}$).

Structurally, slo1 (alpha) subunits can be divided into three major domains: The voltage-sensing domain (VSD), including transmembrane segments S1-S4, which senses the voltage; a pore-gate domain (PGD), including S5-S6, that controls ion permeation and selectivity; and a C-terminal cytoplasmic domain (CTD) that includes two Regulators of potassium conductance (RCK) domains, which recognizes intracellular ligands such as $\mathrm{Ca}^{2+}, \mathrm{Mg}^{2+}$. In addition, unique to BK channel, there is an additional transmembrane domain, S0 that presents N-terminus to the extracellular side of the membrane (Figure 1-2). In most tissues, $\alpha$ subunits are associated with relatively small regulatory termed $\beta$ subunits ( $\beta 1$ - $\beta 4$ ) (Latorre et al. 1989; Behrens et al. 2000; Brenner et al. 2000; Salkoff et al. 2006; Cheng and Lederer 2008; Horrigan 2012).

\subsubsection{Beta Subunits of BK Channels}

While basic characteristics of BK channels (such as voltage- and calciumsensitivity, high conductance) are reproduced by functional tetramers composed from slo1 subunits (Liu et al. 2006; Lee and Cui 2010; Horrigan 2012), some biophysical and pharmacological properties are drastically modified by dynamic association of BK $\alpha$ subunit with regulatory $\beta$ subunits. Beta subunits themselves do not form functional channels per se, rather they are thought to be associated with alpha subunits in a 1:1 ratio (Figure 1-2) (Wallner et al. 1995). 


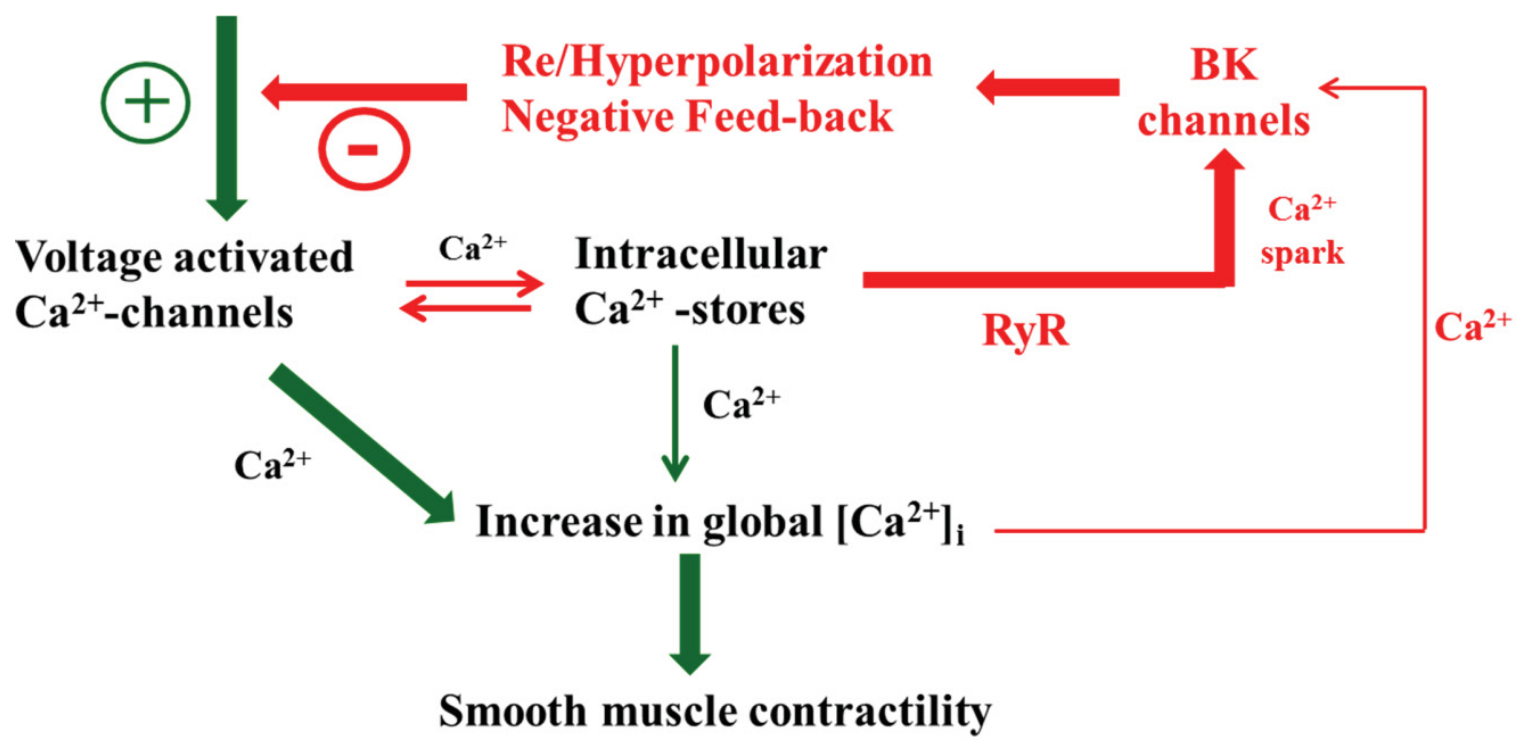

Figure 1-1. Role of BK channels in regulation of vascular tone

Notes: A) Schematic diagram showing that in arterial smooth muscle cells, activation of BK channels provides negative feedback on voltage-dependent calcium influx, limiting the smooth muscle contractility. Membrane depolarization leads to activation of voltagedependent $\mathrm{Ca}^{2+}{ }_{\mathrm{i}}$ channels (VDCC), thus increases the levels of global $\mathrm{Ca}^{2+}{ }_{\mathrm{i}}$. That provides major contribution for smooth muscle contractility. Increased intracellular $\mathrm{Ca}^{2+}{ }_{\mathrm{i}}$ leads to $\mathrm{Ca}^{2+}{ }^{2}$-induced $\mathrm{Ca}^{2+}{ }_{i}$ release from intracellular $\mathrm{Ca}^{2+}{ }_{i}$ stores. This in turn activates nearby BK channels to provide negative feedback for smooth muscle contractility.

Source: Modified with permission from Cheng H. Lederer WJ (2008). Calcium sparks. Physiol Rev 88:1491-1545. 


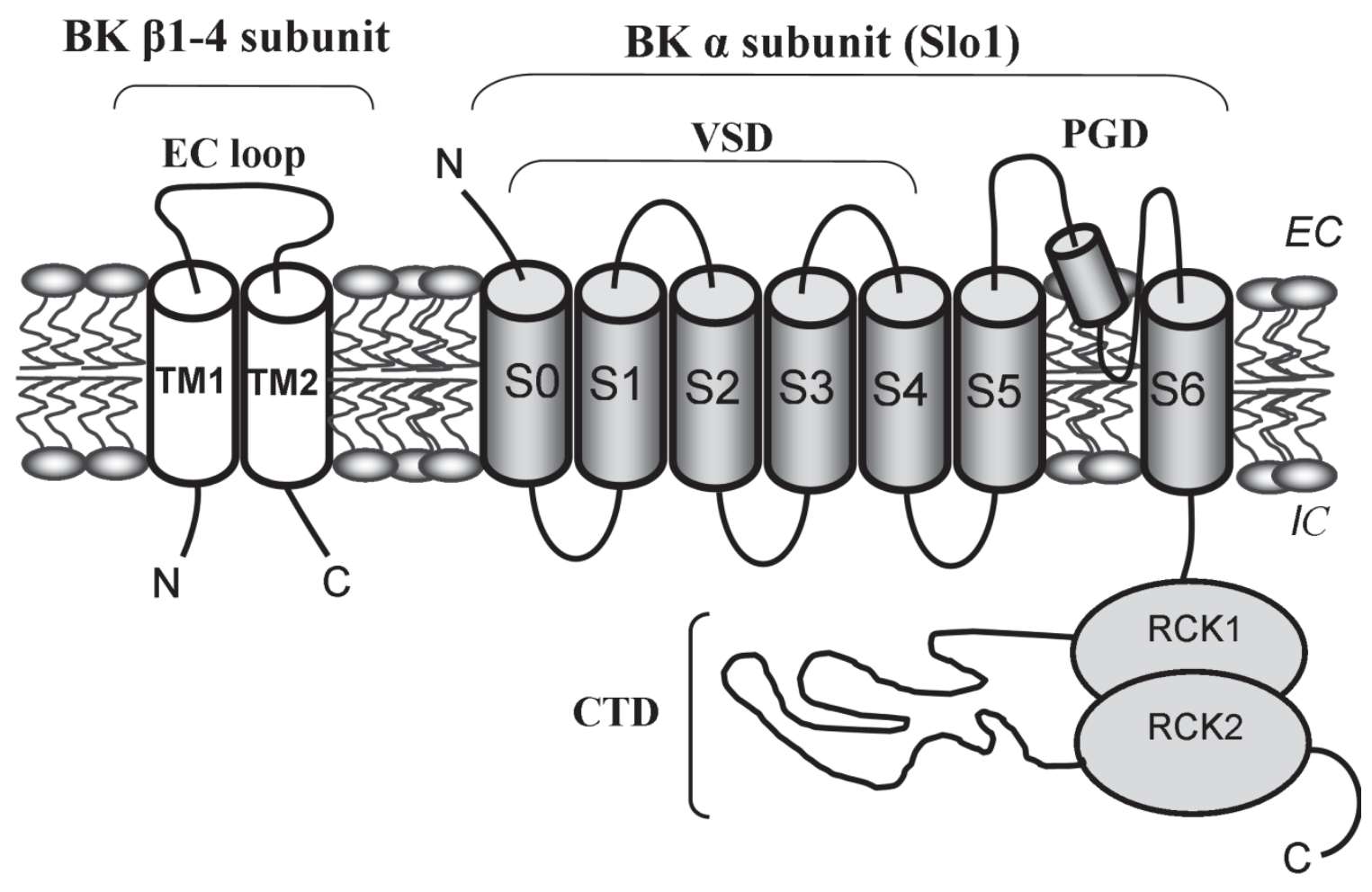

Figure 1-2. Schematic structure of BK channels containing accessory subunit ( $\beta 1$ 4)

Notes: Cartoon showing an $\alpha$ or slo1 (cbv1)- $\beta 1$ heterodimer. The slo1 monomer shows N-terminus, transmembrane (TM) segments from S0-S6 and intracellular regulator of conductance for $\mathrm{K}^{+}$(RCK) domains. Among slo1 TM domains, S0-S4 domains forms voltage sensing domain (VSD), which senses changes in membrane potential; S5-S6 domains involved in forming pore of the channel, called pore-gate domain (PGD). The RCKs, contains recognition sites for intracellular ligands such as $\mathrm{Ca}^{2+}{ }_{\mathrm{i}}$ and $\mathrm{Mg}^{2+}$. Four slo1 monomers associate to render functional channel forming BK channels. $\beta$ subunits contain an N-terminal, two transmembrane domains (TM1 and TM2), extracellular (EC) loop, and a C-terminal. EC and IC correspond to the extracellular and intracellular edges of the membrane; RCK: Regulator of Conductance for $\mathrm{K}^{+}$domain.

Source: Reprinted with permission from Kuntamallappanavar G. Toro L. and Dopico A.M. (2014). Both transmembrane domains of BK beta1 subunits are essential to confer the normal phenotype of beta1-containing BK channels. PLoS One 9(10): e109306. 
$\beta$ subunits are characterized by differential distribution among the tissues (Behrens et al. 2000; Brenner et al. 2000). Of the four $\beta$ subtypes, $\beta 1$ is the predominant isoform in vascular smooth muscle (Tanaka et al. 1997; Brenner et al. 2000; Cox and Aldrich 2000). $\beta 1$ subunit drastically increases the apparent $\mathrm{Ca}^{2+}$ sensitivity of the channel, and slows down activation and deactivation kinetics (Brenner et al. 2000; Cox and Aldrich 2000). $\beta 2$ is expressed in adrenal gland and brain (Behrens et al. 2000) and characterized by the presence of an intracellular inactivating domain that empowers the ionic current with fast inactivation (Brenner et al. 2000). $\beta 3$ is predominant in testis and skeletal muscle (Uebele et al. 2000). However, it does not alter calcium $\left(\mathrm{Ca}^{2+}{ }_{\mathrm{i}}\right)$ sensitivity, activation or deactivation kinetics (Brenner et al. 2000). $\beta 4$ is abundantly expressed in brain. $\beta 4$-containing BK channels are characterized by 1 ) resistance to the BK peptidic blockers iberotoxin (Ibtx) and charybdotoxin (Ctx) due to the presence of a large extracellular loop; and 2) slow activation and deactivation kinetics (Brenner et al. 2000).

From a structural point of view, beta subunits consists of two transmembrane domains (TMs) interconnected by a long extracellular (EC) loop and short cytoplasmic N- and C-termini (Figure 1-2) (Behrens et al. 2000; Brenner et al. 2000; Cox and Aldrich 2000).

\subsubsection{Role of BK $\beta 1$ Subunit in Regulation of Arterial Tone}

BK $\beta 1$ serves as a key molecule that enables activation of BK channels in response to micromolar $\mathrm{Ca}^{2+}{ }_{i}$ concentration released from the sarcoplasmic reticulum. $\beta 1$ is known to increase BK channel's apparent $\mathrm{Ca}^{2+}{ }_{\mathrm{i}}$ sensitivity (Meera et al. 1996; Tanaka et al. 1997; Brenner et al. 2000; Brenner et al. 2000; Cox and Aldrich 2000; Patterson et al. 2002). $\beta 1$ knock-out mice suffer from a variety of abnormalities due to impaired vasorelaxation mechanisms. These abnormalities are linked to BK channel function in cardiovascular system leading to hypertension, stroke, brain hemorrhage and cardiac hypertrophy without major alterations involving BK in other tissues or organs (Brenner et al. 2000; Pluger et al. 2000). Interestingly, BK channels from $\beta 1$ knock-out mice lack response to $\mathrm{Ca}^{2+}{ }_{i}$ sparks, a vasodilating signal regenerated by the activity of ryanodine receptors. Furthermore, these studies have also demonstrated that cerebral arteries from $\beta 1$ knock-out mice constrict more than wt with mean arterial blood pressure of $\beta 1$ knockout mice being significantly elevated in comparison to control (Brenner et al. 2000; Lohn et al. 2001; Patterson et al. 2002). Notably, genetic hypertensive rats show down regulation of BK $\beta 1$, normal spark amplitude but reduced STOC amplitude, underscoring the importance of BK $\beta 1$ in spark-STOC coupling in vascular smooth muscle (Amberg and Santana 2003). 


\subsection{Alcohol and BK Channels}

\subsubsection{Ethanol Modulation of BK Channel Activity}

It was first documented in mid 90's that acute ethanol exposure (10-100 mM) increases the BK steady-state activity; in turn contribute to alcohol-induced inhibition of vasopressin, oxytocin, and growth hormone release (Dopico et al. 1996; Jakab et al. 1997; Dopico et al. 1998; Dopico 2003). In addition, BK channel activation by ethanol has been also reported in several other preparations and further linked to pathophysiological processes that are affected by ethanol exposure, such as neurotransmitter release, nociception, action potential firing in N.accumbens neurons, motor coordination in C.elegans, endothelial proliferation and nitric oxide production (Brodie et al. 2007) However, ethanol-induced BK current activation is not a universal phenomenon, with BK channels in rat supraoptic neuron somata (Dopico et al. 1999) and in N. Accumbens dendrite being refractory to $100 \mathrm{mM}$ ethanol (Martin et al. 2004). Moreover, ethanol drastically inhibits the BK channels in rat aorta (Walters et al. 2000), and cerebral arteries from rat and mouse, eventually leading to cerebral artery constriction (Liu et al. 2004; Bukiya et al. 2009; Bukiya et al. 2011).

\subsubsection{Factors Modulating Ethanol Action of BK Channel Activity}

Previous studies demonstrated that the final ethanol effect on BK channel activity is a result of the orchestration of several factors such as: 1. post-translational modification (phosphorylation) of pore-forming slo1 subunit (Liu et al. 2006); 2. co-expression of channel's auxiliary subunits (Martin et al. 2004; Bukiya et al. 2009; Wynne et al. 2009); 3. amount of cholesterol in the membrane where the ion channel resides (Crowley et al. 2003; Crowley et al. 2005; Bukiya et al. 2011; Yuan et al. 2011); 4. phospholipid species in the membrane surrounding channel (Crowley et al. 2005; Yuan et al. 2011).

Collectively, results from reductionist systems such as reconstitution of recombinant slo1 protein in artificial bilayer revealed that ethanol action on BK channels requires two minimum set of targets i.e., slo1 subunit itself and one or two phospholipid species in the bilayer (Crowley et al. 2005; Brodie et al. 2007; Yuan et al. 2011). Whether final ethanol effect of ethanol on BK channel is potentiation or inhibition, modulation of BK channel activity in response to acute ethanol exposure is ultimately determined by modification of slo1 channel gating processes, without modification in channel expression or unitary conductance (Dopico et al. 1996; Dopico et al. 1998; Dopico 2003; Martin et al. 2004). Thus, ethanol effect on BK current is limited to gating modifier.

Slo1 channel gating is defined by four basic processes: 1 . voltage-driven gating 2 . $\mathrm{Ca}^{2+}$-driven gating $3 . \mathrm{Mg}^{2+}$-driven gating 4. intrinsic gating (Cox and Aldrich 2000). Thus, ethanol may alter one or more of these gating processes to modify the steady-state activity of the channel and eventually perturb cell physiology and organ function. 


\subsubsection{Role of BK $\beta 1$ Subunit in Ethanol-Induced Cerebral Vasoconstriction}

Several studies demonstrated that ethanol action on arterial smooth muscle leads to ethanol-induced cerebral vasoconstriction (Altura and Altura 1982; Altura et al. 1983; Altura and Altura 1984; Gordon et al. 1995; Yang et al. 2001; Liu et al. 2004). Our laboratory documented that the cerebral arterial constriction by ethanol concentrations obtained during binge drinking is due to ethanol-induced inhibition of smooth muscle BK channel, as it is vanished in the presence of the selective BK channel blocker iberiotoxin (Ibtx) with blockers of other voltage-dependent $\mathrm{K}^{+}$channels failing to modulate ethanol action (Liu et al. 2004). Remarkably, BK $\beta 1$ subunit is required for ethanol-induced cerebral artery constriction (Bukiya et al. 2009): indeed, using the $K C N M B 1 \mathrm{~K} / \mathrm{O}$ mice model, we demonstrated that BK channel-mediated ethanol-induced vasoconstriction is not observed in the absence of BK channel $\beta 1$ subunits (Bukiya et al. 2009). Furthermore, this finding was paralleled by patch-clamp recordings of native BK channels from freshly isolated cerebral artery myocytes showing that $\mathrm{BK}$ channels from $w t$ myocytes are inhibited by ethanol whereas, BK channels in $K C N M B 1 \mathrm{~K} / \mathrm{O}$ myocytes were resistant to ethanol inhibition. Additionally, native BK channels from skeletal muscle fibers, where $\beta 1$ is poorly, if at all, expressed, were also resistant to ethanolinduced inhibition (Bukiya et al. 2011).

\subsection{Significance of the Study}

Episodic, moderate-to-heavy alcohol intake leads to cerebral artery constriction, vasospasm, and stroke, which are primarily determined by ethanol action on cerebral artery smooth muscle itself. In addition, it was demonstrated that the BK channel in cerebrovascular smooth muscle is the major cell effector of ethanol-induced cerebral artery constriction and its inhibition by ethanol action is favored by the expression of the smooth muscle-abundant regulatory $\beta 1$ subunit (Liu et al. 2008; Bukiya et al. 2009). Alcohol-induced constriction ( $\sim 10 \%$ decrease in diameter) is sufficient to decrease the cerebral blood flow by $\sim 30 \%$ because diameter and blood flow are related by a $3^{\text {rd }}$ power relationship.

Considering the high prevalence of binge drinking and cerebrovascular disease, the association between alcohol intake and cerebrovascular pathology and the high mortality and disability that result from cerebrovascular disease, it is crucial to identify the molecular targets and mechanisms that mediate ethanol-driven cerebrovascular disorders. Although, pharmacotherapy (e.g., accamprosate, naltrexone) or other forms of approaches are aimed at treating alcohol-driven disorders by breaking the circuit of addiction, however, none of these therapies are aimed to prevent or ameliorate the cerebrovascular disorders related to binge drinking and acute ethanol intoxication.

Specific aims in this proposal are designed to identify the specific region(s) in the $\beta 1$ subunit that are responsible for ethanol-induced BK channel inhibition and resulting cerebrovascular constriction as well as the specific gating mechanisms that lead to ethanol-inhibition of $\beta 1$-containing BK channel currents . Knowing which specific 
region(s) of $\beta 1$ subunit and the specific gating processes that are targeted by ethanol to disrupt BK channel function and thus evoke cerebrovascular constriction could help to develop pharmacological agents/tools that would counteract these ethanol actions by acting on specific BK region/gating processes. Should these counteracting agents be found free of side effects and suitable to human pharmacokinetics, they could be used in our pharmaco-therapeutic arsenal to oppose ethanol-driven cerebrovascular constriction and disease. 


\section{CHAPTER 2. HYPOTHESIS}

\subsection{Goal}

The overall goal of this study is to identify the molecular targets that underlie ethanol-induced inhibition of $\beta 1$-containing BK channels and the resulting cerebral artery constriction. I will reach this goal by addressing the following specific aims.

\subsection{Specific Aims (SA)}

\subsubsection{Specific Aim 1}

Determine the role of activating ion and accessory beta subunits in ethanol modulation of BK channels.

\subsubsection{Specific Aim 1 (Part-A)}

Determine the role of activating ion in ethanol modulation of BK channels.

After documenting that ethanol modulation of BK channel activity is a function of the channel's activating ion, i.e. intracellular $\mathrm{Ca}^{2+}{ }_{\mathrm{i}}$ (Liu et al. 2008), I expressed BK channel pore-forming $\alpha$ subunits cloned from rat cerebral artery myocytes (termed cbv1; AY330293) in Xenopus oocyte expression system. This slo1 variant showed all properties of other BK channel-forming slo1 proteins, including its independent gating by $\mathrm{Ca}^{2+}{ }_{\mathrm{i}}$ and $\mathrm{Mg}^{2+}{ }_{\mathrm{i}}$. Using the same expression system, I also expressed other slo family members i.e. slo2 and slo3, which are primarily gated by ions other than $\mathrm{Ca}^{2+}$ : i.e., $\mathrm{Na}^{+}$ and $\mathrm{OH}^{-}$respectively. Then, I used patch-clamp electrophysiology to determine the role of activating ion in ethanol modulation of the different members of the slo channel family. Part-A of SA1 is addressed in Chapter 4.

\subsubsection{Specific Aim 1 (Part-B)}

Determine the role of different BK $\beta$ subunits in ethanol modulation of BK channels.

Previous studies have identified that BK $\beta$ subunits modulate the apparent $\mathrm{Ca}^{2+}{ }_{\mathrm{i}}$ sensitivity of the channel (Brenner et al. 2000; Cox and Aldrich 2000), this modulation likely contributing to ethanol-induced inhibition of $\beta 1$-containing BK channels. These channels are thought to constitute native channel, in cerebral artery smooth muscle, which mediate cerebral artery constriction by ethanol (Liu et al. 2004; Bukiya et al. 2009). Thus, I co-expressed cbv1 with different BK $\beta$ subunits and used patch-clamp 
electrophysiology to determine the role of different accessory $\beta$ subunits in ethanol modulation of BK channel. Part-B of SA1 is addressed in Chapter 5.

\subsubsection{Specific Aim 2}

Identify the specific $\beta 1$ subunit-dependent gating processes that are targeted by ethanol leading to modification of BK currents.

Several groups have documented that BK $\beta 1$ subunits increase the BK channel's apparent $\mathrm{Ca}^{2+}{ }_{\mathrm{i}}$ sensitivity (Brenner et al. 2000; Cox and Aldrich 2000; Bao and Cox 2005; Orio and Latorre 2005). This $\beta 1$ action, however, results from complex modulation of various specific aspects of BK channel gating, some being $\mathrm{Ca}^{2+}{ }_{i}$-dependent while others being $\mathrm{Ca}^{2+}{ }_{\mathrm{i}}$-independent: 1) mild increase in the $\mathrm{Ca}^{2+}$ affinity to closed state(s) $\left(\mathrm{K}_{\mathrm{d}}\right)$ of the channel; 2) decrease in the intrinsic energetic barrier of the channel to transition from closes to open states in absence of any ligand or transmembrane voltage (i.e., $\mathrm{L}_{0}=$ closed to open equilibrium constant) (Cox and Aldrich 2000; Bao and Cox 2005; Orio and Latorre 2005).3) stabilization of voltage sensor activation $\left[\mathrm{V}_{\mathrm{h}}(\mathrm{J})\right]$, when the channel is closed. 4) Mild increases in allosteric interaction (factor C) between $\mathrm{Ca}^{2+}{ }_{\mathrm{i}}$ binding and channel opening and thus promotes channel opening (Cox and Aldrich 2000; Bao and Cox 2005). Based on this background information, I conducted normalized macroscopic conductance $\left(\mathrm{G} / \mathrm{G}_{\mathrm{max}}\right)$-voltage $(\mathrm{V})$ curves obtained over a wide range of $\left[\mathrm{Ca}^{2+}\right]_{\mathrm{i}}$ from homomeric cbv1 vs. cbv1+ $\beta 1$ constructs under condition similar to those proposed to test SA1. The resulting plots were fitted to the 70-state Horrigan-Aldrich (HA) allosteric model (Horrigan and Aldrich 1999; Horrigan and Aldrich 2002; Orio and Latorre 2005; Sun et al. 2013; Gonzalez-Perez et al. 2014) to determine which specific aspects of channel gating were modified by ethanol $(50 \mathrm{mM})$ and led to inhibition of $\beta 1$ containing BK channel currents. SA2 is addressed in Chapter 5.

\subsubsection{Specific Aim 3}

Identify the specific $\beta 1$ subunit region(s) that is responsible for ethanol-induced inhibition of $\beta 1$-containing BK channels.

Previous studies have demonstrated that BK $\beta 1$ subunit enables ethanol-induced BK channel inhibition at physiological ionic conditions whereas BK $\beta 4$ subunit does not contribute to this ethanol action (Liu et al. 2008; Bukiya et al. 2009). Based on these findings, I co-expressed cbv1 with chimeric subunits made by swapping different regions between BK $\beta 1$ and BK $\beta 4$. Then, I used patch-clamp electrophysiology on the resulting heteromeric BK complexes in the same expression system and conditions previously used to study cerebral artery recombinant BK channel function. The strategy was to investigate ethanol action on BK channel complexes containing $\beta 1 / \beta 4$ chimeras where the swapped region was progressively smaller. Therefore, I narrowed down the specific region(s) $(\mathrm{C}$ end, TM1, EC loop, TM2, N-end) in the BK channel $\beta 1$ subunit that was responsible for 
ethanol-induced decrease in BK channel steady-state activity. SA3 is addressed in Chapter 6.

\subsubsection{Specific Aim 4}

Address the involvement of the $\beta 1$ subunit region(s) that mediates ethanolinduced BK channel inhibition in alcohol-induced cerebral artery constriction

After identifying the specific region in the BK $\beta 1$ subunit that is responsible for ethanol-induced BK channel inhibition, I used a combination of reversible permeabilization and isolated artery pressurization to determine the contribution of such specific subunit domain to ethanol-induced cerebral artery constriction. Thus, I applied $50 \mathrm{mM}$ ethanol to middle cerebral arteries isolated from $K C N M B 1$ knock-out mouse, these arteries being previously transfected with $w t \beta 1$ or different chimeric $\beta 1 / \beta 4$ proteins. Arteries from $K C N M B 1$ knock-out transfected with $w t \beta 1$ and arteries from $w t$ (C57BL/6) mouse served as positive controls for ethanol action while arteries from $K C N M B 1$ knock-out mice and $K C N M B 1$ knock-out mice arteries transfected with empty vector served as negative controls. SA4 is addressed in Chapter 6. 


\section{CHAPTER 3. MATERIALS AND METHODS}

\subsection{Vertebrate Animals}

\subsubsection{Ethical Aspect of Research}

The animal care and use of animals, and performed research were conducted in accordance with policies from the Institutional Animal Care and Use Committee (IACUC) at the University of Tennessee Health Science Center (UTHSC) and provision of animal welfare act (USDA). UTHSC is an Institution accredited by the Association for Assessment and Accreditation of Laboratory Animal Care (AAALAC). Our AAALACaccredited facility is staffed with three full-time veterinarians, two of them being boardcertified in laboratory animal care. The veterinary care of all vertebrate animals on campus was handled by the Department of Comparative Medicine (DCM). DCM meets all applicable federal and state laws on animal care, and fully complies with the Publich Health Service (PHS) policy on Humane Care and Use of Laboratory Animals. In order to minimize the transmission of infectious diseases, animals were housed by health status and species.

\subsubsection{Information of Veterinary Care of Animals}

Xenopus leavis frogs were maintained in aquaria containing artificial pond water and fed with calf's liver and a nutritionally balanced frog brittle to satiation twice a week. The health of frogs under these conditions was very good. The health status of the frogs, prior surgery was performed following standard evaluations, such as swimming behavior, appetite and amount of shedding.

Mice (wt CL57/B or KCNMB1 knock-out) were housed on a 12 hour light/dark cycle, with water and pellet food ad libitum. Their health status prior experimentation was performed following standard evaluations, such as appearance, behavior, activity, appetite, excreta, and weight.

\subsubsection{Procedures to Minimize Discomfort, Distress, Pain and Injury}

Frogs were anesthetized with hypothermia (ice water) prior to surgery. Immediately after surgery and suturing, frogs are immersed in pond water containing MS-222 (200-300 mg/l, according to frog size) as anesthetic for 5 minutes, and the animal body temperature is brought gradually back to room temperature to avoid shock. Then, animals were switched back to pond water. Frogs recovered quickly from surgery with no complications. 
Several hours before decapitation, mice are housed in the lab where the procedure was performed. They were handled several times by the operator before being killed.

These simple measures minimized the stress experienced by the animal before the actual experiment.

\subsubsection{Method of Euthanasia}

When the removal of ovarian material was complete, anesthetized animals with MS-222 were euthanized by decapitation. The method is consistent with the recommendations of the Panel of Euthanasia of the American Veterinary Medical Association (AVMA).

Mice were euthanized by decapitation using sharp scissors. The method is also consistent with the recommendations of the Panel of Euthanasia of the AVMA.

\subsection{Cloning of cDNA and Transcription}

\subsection{1. cDNA Coding for BK Channels}

Cerebral artery smooth muscle cell slo1 isoform, cbv1 (AY330293) and associated $\beta 1$ (FJ154955) cDNA were cloned from rat cerebral arteries. Briefly, first, basilar and middle cerebral arteries were dissected out from rat brain on ice. Smooth muscle cells from these arteries were isolated and identified under inverted microscope using Hoffman optics. Individually isolated myocytes were aspirated in to Eppendorf tubes containing approximately $50 \mu \mathrm{L}$ PicoPure extraction buffer (Arcturus). Total RNA from approximately 100 myocytes was obtained using PicoPure kit (Arcturus). Then, reverse transcription of first strand cDNA was carried out using superscriptII reverse transcriptase with oligo(dT) primer (Invitrogen) (Jaggar et al. 2005). Cbv1 cDNA inserted into the pBScMXT vector was linearized with SalI and transcribed in vitro using T3 polymerase (mMessage-mMachine; Ambion, Austin, TX). Cbv1 cDNA inserted into pBScMXT is cleaved by BamHI (Invitrogen) and XhoI (Promega), and directly reinserted into $\mathrm{pOX}$ for expression in $X$. laevis oocytes. pOX vector-containing cbv1 was linearized with NotI (Promega) and then transcribed in vitro using T3 polymerase.

Slo2.1, slo 2.2, and slo3 cDNAs were generous gifts from Len Kaczmarek (Yale University School of Medicine, New Haven, CT) and Larry Salkoff (Washington University School of Medicine, St. Louis, MO). These constructs were recloned into pOX vector for oocyte expression. The cDNA-containing vectors were linearized with NotI and were transcribed using T3 polymerase and the mMessage mMachine kit (Ambion, Austin, TX) (Liu et al. 2013). 


\subsection{2. cDNA Coding for BK Channel Accessory Beta Subunits}

BK $\beta 1$ cDNA cloned into the EcoR I and Sal I sites of the pCI-neo vector was linearized with Not I and transcribed using T7. Then, BK $\beta 1$ cDNA was re-cloned into pOX vector for oocyte expression. pOX- $\beta 1$ was then linearized and transcribed in vitro using either T7 polymerase (mMessagemMachine; Ambion, Austin, TX) (Bukiya et al. 2009; Bukiya et al. 2013).

Wt $\beta 2$ cDNA in $\mathrm{pXMX}$ vector was a generous gift from Christopher Lingle (Washington University School of Medicine, St. Louis, MO) linearized with MluI and transcribed with T7. $\beta 2$ subunit with the inactivation bowl removed ( $\beta 2$-IR), $\beta 3, \beta 4$, and $\beta 1 / \beta 4$ chimeric cDNAs $\left(\beta_{1} \mathrm{TMs}_{4}, \beta_{4} \mathrm{TMs}_{1}, \beta_{4} \mathrm{TM} 1_{1}\right.$ and $\left.\beta_{4} \mathrm{TM} 2_{1}\right)$ were generous gifts from Ligia Toro (University of California, LA). $\beta 2$-IR in $\mathrm{pXMX}$ vector was linearized with NotI and transcribed with T7. $\beta 3$ cDNA inserted into the pOX and was linearized with SalI and transcribed with T7. $\beta 4$ cDNA was subcloned into pOX vector and linearized by NotI and transcribed using T3. $\beta 1 / \beta 4$ chimeric cDNAs were inserted into $\mathrm{pOX}$ vectors. Linearization of $\mathrm{pOX}$ vectors containing chimeric $\beta 1 / \beta 4$ was carried out with NotI and linear cDNAs were transcribed using T3. $\beta 1 / \beta 4$ chimeras by swapping different domains of $\beta 1$ and $\beta 4$ in such a way that swapped region became progressively smaller.

Different $\beta 1 / \beta 4$ chimeras we used were: $1 . \beta_{1} \mathrm{TMs}_{4}$, which contained $\mathrm{h} \beta 4 \mathrm{TMs}$ on $\mathrm{h} \beta 1$ "background" (i.e., h $\beta 1$ EC loop and IC ends); 2. $\beta_{4} \mathrm{TMs}_{1}$, which contained $\mathrm{h} \beta 1 \mathrm{TMs}$ on h $\beta 4$ "background" (i.e., h $\beta 4$ EC loop and IC ends); $3 . \beta_{4} \mathrm{TM} 1_{1}$, which contained $\mathrm{h} \beta 1$ TM1 on h $\beta 4$ "background" (i.e., h $\beta 4$ TM2, EC loop and IC ends); 3. $\beta_{4} \mathrm{TM} 2{ }_{1}$, which contained h $\beta 1$ TM2 on h $\beta 4$ "background" (i.e., h $\beta 4$ TM1, EC loop and IC ends); 4. $\beta_{4} \mathrm{~L}_{1}$, which contained EC loop from $\mathrm{h} \beta 1$ on $\mathrm{h} \beta 4$ "background" (i.e., $\mathrm{h} \beta 4 \mathrm{TMs}$ and IC ends; 5 . $\beta_{1} \mathrm{~L}_{4}$, which contained EC loop from $\mathrm{h} \beta 4$ on $\mathrm{h} \beta 1$ "background" (i.e., h $\beta 1 \mathrm{TMs}$ and IC ends).

All cDNA sequences were confirmed by automatic sequencing at the UTHSC Molecular Research Center.

\subsection{Oocyte Isolation from Xenopus Leavis and RNA Injection}

\subsubsection{Model Justification: Xenopus Leavis Oocytes}

We choose Xenopus laevis oocytes to heterologously express BK channel because: 1) these cells express negligible amount of endogenous BK channels; 2) they contain endogenous ion channels (primarily mechano-gated cation and $\mathrm{Cl}^{-} \mathrm{Ca}$ channels (Miledi and Parker 1984; Meissner et al. 1989) that can be easily differentiated from BK channels based on conduction and gating; 3 ) by varying amount of cRNA and interval between cRNA injection and patch-clamping, we can control amount of channel expression; 4) using oocytes, we already characterized ethanol effect on cbv1 and cbv1+ $\beta 1$ channels (Bukiya et al. 2009); 5) we functionally expressed and characterized 
the heteromeric channels containing chimeric $\beta$ s that are necessary to address SA3 (Bukiya et al. 2009); 6) we collected lithocholate (LCA) data on cbv1 and cbv1+ $\beta$ s (Bukiya et al. 2007; Bukiya et al. 2008; Bukiya et al. 2009). LCA and Ibtx are pharmacological tools that we will use to confirm the functional presence of $\beta 1-, \beta 4-$, and $\beta 1 / \beta 4$ chimera-containing BK channels; 8) Dr.Dopico and his personnel have $>20 \mathrm{yr}$. experience with the oocyte model.

\subsubsection{Oocyte Isolation from Xenopus Leavis Oocytes}

Female Xenopus laevis frogs were purchased from Xenopus Express (Brooksville, FL). They were maintained in artificial pond water on a 12-hour light/dark cycle. In this environment, frogs do not show the behavior of seasonal breeding, thus oocytes were usually available throughout the year. Stages V and VI oocytes were predominantly collected because oocytes at these particular stages transcribe mRNA into protein/channels with higher efficiency. Surgical procedure was as follows: First, frogs were anesthetized by placing them on ice after exposure to ethyl 3-aminobenzoate methanesulfonate salt (MS-222) (250 mg/l, pH 7.4). A small surgical incision (of $\sim$ less than $1 \mathrm{~cm}$ ) was made in the abdomen and the oocytes were extracted. Extracted oocytes were kept in a $\mathrm{Ca}^{2+}$-free ND-96 solution (mM): $82.5 \mathrm{NaCl}, 2 \mathrm{KCl}, 1 \mathrm{MgCl}_{2}, 5$ HEPES, $\mathrm{pH} 7.4$, which contains $2 \mathrm{mg} / \mathrm{ml}$ collagenase (type IV; Sigma-Aldrich), at room temperature on a horizontal shaker (70 oscillations per minute) for 15-20 minutes to remove the follicular layer. After defolliculation, the individually isolated oocytes were transferred to $\mathrm{Ca}^{2+}$-containing ND-96 solution (mM): $82.5 \mathrm{NaCl}, 2 \mathrm{KCl}, 1.8 \mathrm{CaCl}_{2}, 5$ HEPES, pH 7.4, supplemented with $2.5 \mathrm{mM}$ sodium pyruvate and 10,000 I.U. of penicillin and $10 \mathrm{mg}$ of streptomycin in $500 \mathrm{ml}$ (Dopico et al. 1996; Bukiya et al. 2009).

\subsection{3. cRNA Injection}

cRNA was dissolved in diethyl polycarbonate-treated water at 10 (cbv1/slo2.1 and slo2.1/slo3) or 30 ( $\beta \mathrm{s}$ or engineered $\beta \mathrm{s}$ ) $\mathrm{ng} / \mu \mathrm{l} ; 1 \mu \mathrm{l}$ aliquots are stored at $-80^{\circ} \mathrm{C}$. Cbv1; slo2.1/slo2.2; and slo3 cRNAs alone were injected approximately about $10 \mathrm{ng} / \mu 1$. On the other hand, co-injection of cbv1 (10ng/ $\mu 1)$ with $\beta 1, \beta 2, \beta 2$-IR, $\beta 3, \beta 4$, or engineered $\beta$ (30ng/ $\mu \mathrm{l}$ ) cRNAs was conducted to achieve molar ratios of $\geq 6: 1$ ( $\beta: \alpha)$ to ensure that all cbv1 subunits are saturated with $\beta$ s (Dopico 2003; Liu et al. 2008; Bukiya et al. 2009). cRNA of $\sim 23 \mathrm{nl} /$ cell was injected into the oocyte cytoplasm using a drummond nanoinjector (Drummond Scientific Co., Broomall, PA). The interval between cRNA injection and patch-clamp electrophysiology recording was approximately 36 to 48 hours. During this time period, the injected oocytes were incubated at $14-16^{\circ} \mathrm{C}$. 


\subsection{Patch-Clamp Electrophysiology}

\subsubsection{Data Acquisition}

Before electrophysiology recordings, oocytes were placed into a petri dish containing a hypertonic solution (mM): $200 \mathrm{~K}^{+}$aspartate, $20 \mathrm{KCl}, 1 \mathrm{MgCl}_{2}, 10$ EGTA, 10 HEPES, $\mathrm{pH} 7.4$, for 15-20 minutes. With this hypertonic treatment, the oocytes shrink, allowing the removal of the vitelline membrane with forceps and thus, exposing the oocyte membrane for subsequent patch-clamp recording. Then, the oocytes were placed into $\mathrm{Ca}^{2+}$-containing ND-96 solution for 10-15 minutes before recording ionic currents. Ionic currents were recorded from excised, inside-out ( $/ / O)$ macro patches. The following paragraphs show the compositions of bath and electrode solutions for recording different constructs.

Homomeric cbv1 and $\beta$ subunit-containing BK channels: the bath and electrode solutions had the same composition (mM): $135 \mathrm{~K}$-gluconate, 5 EGTA, $2.28 \mathrm{MgCl}_{2}, 15$ HEPES, 1.6 HEDTA, pH 7.35 (Liu et al. 2008; Bukiya et al. 2009).

For the experiments in which cbv1 channels were probed with ethanol at different $\left[\mathrm{Mg}^{2+}\right]$, both bath and electrode solutions contained (mM): $140 \mathrm{~K}$-methanesulfonate, 20 HEPES, $2 \mathrm{KCl}, 5$ EGTA; $\mathrm{pH}$ 7.2. The desired $\left[\mathrm{Mg}^{2+}\right.$ i $]$ was obtained by adding $\mathrm{MgCl}_{2}$ (Shi et al. 2002).

Rslo2.1:rslo2.2 channel recording: bath and electrode solutions contained (mM): $130 \mathrm{~K}$-gluconate, 5 EGTA, 10 HEPES, 29 glucose, and variant amounts of Na-gluconate: 10, 70, and $140 \mathrm{mM}$; pH 7.3 (Santi et al. 2006).

Mslo3 channel recording: Intracellular solution contained (mM): $140 \mathrm{~K}-$ methanesulfonate, $20 \mathrm{KOH}, 10 \mathrm{HEPES}\left(\mathrm{H}^{+}\right)$, titrated with methanesulfonic acid or $\mathrm{KOH}$ to $\mathrm{pH}=6.0,7.0,8.0$, or 9.0, which correspond to $\left[\mathrm{OH}^{-}\right]=0.01,0.1,1$, and $10 \mu \mathrm{M}$, respectively. Extracellular solution contained $(\mathrm{mM}): 140 \mathrm{~K}$-methanesulfonate, $20 \mathrm{KOH}$, $10 \mathrm{HEPES}\left(\mathrm{H}^{+}\right)$and $2 \mathrm{MgCl}_{2}$, titrated with methanesulfonic acid to $\mathrm{pH}=7.0\left(\left[\mathrm{OH}^{-}\right]=0.1\right.$ $\mu \mathrm{M})$ (Xia et al. 2004).

In all patch-clamp electrophysiology studies that require tight buffering of $\mathrm{Ca}^{2+}$ in solution, the free $\mathrm{Ca}^{2+}$ in solution was adjusted to the desired value by adding $\mathrm{CaCl}_{2}$. When the desired free $\mathrm{Ca}^{2+}$ did not exceed $1 \mu \mathrm{M}$, HEDTA was omitted from the solution. The final concentration of the $\mathrm{MgCl}_{2}$ was set to $1 \mathrm{mM}$. In all experiments, nominal free $\left[\mathrm{Ca}^{2+}\right]$ is calculated using MaxChelator (WEBMAXC, Stanford University, Stanford, $\mathrm{CA}$ ) and experimentally validated using $\mathrm{Ca}^{2+}$-selective electrodes (Corning Incorporated Science Products Division, Corning, NY) (Dopico 2003). The use of gluconate- as main anion substituting for $\mathrm{Cl}^{-}$allows minimizing the contamination of recordings by the activity of endogenous $\mathrm{Cl}^{-}$channels. 
We chose a wide range of $\mathrm{Ca}^{2+}$ concentration i.e $0-100 \mu \mathrm{M}$ at the intracellular side of the excised membrane patch because this range covers the $\mathrm{Ca}^{2+}$ concentration estimated for the vicinity of BK channel both at resting condition and during cerebral artery myocyte contraction (Perez et al. 2001).

Immediately before experiments, patch-clamp electrodes were pulled from glass capillaries (Drummond Scientific Co.). Then, the tip of the electrode was fire-polished on a microforge (World Precision Instruments, Sarasota, FL) to achieve resistance of 2-6 $\mathrm{M} \Omega$ for macroscopic current recording and 8-10 $\mathrm{M} \Omega$ for single channel recording.

We chose I/O macro-patch recordings because: 1) I/O patches allow us to probe ethanol with tightly buffered $\left[\mathrm{Ca}^{2+}\right]$ at the cytosolic side of the channel, controlling BK activity and its $\mathrm{Ca}^{2+}$-dependent modulation by ethanol; 2) it is easier to freshly dilute ethanol in bath solution and apply it to the inner leaflet than perfusing a patch-pipette (as $\mathrm{O} / \mathrm{O}$ or whole-cell recording would require to modify internal $\left.\mathrm{Ca}^{2+}\right)$. Consistent with ethanol fast crossing of the cell membranes, BK response is identical whether ethanol is applied to the inner or outer leaflet (Dopico et al. 1996; Dopico et al. 1998; Dopico et al. 1999; Dopico 2003); 3) macroscopic currents are routinely used to conduct pharmacological studies on BK channel by us and others (Brenner et al. 2000; Cox and Aldrich 2000; Bukiya et al. 2009).

We chose an ethanol concentration of $50 \mathrm{mM}$ because it is reached in blood circulation after episodic moderate-heavy drinking, and linked to cerebrovascular pathology in humans (Moore and Pearson 1986; Marmot and Brunner 1991; Zakhari 1997; Suter and Vetter 1999; Gil-Nunez and Villanueva 2001; Reynolds et al. 2003). Additionally, our previous studies demonstrating a direct ethanol action on native channels were conducted at this [EtOH] (Liu et al. 2004; Bukiya et al. 2009; Bukiya et al. 2011), allowing comparison and proper interpretation of the new data obtained with recombinant channel protein. We choose equimolar concentration of urea as a control to isosmotically replace ethanol.

After excision from the cell, the membrane patch was consecutively exposed to a stream of bath solution containing control (50 mM urea) then $50 \mathrm{mM}$ ethanol, then control (with urea) as washout. Solutions were applied onto the patch cytosolic side via a micropipette tip with an internal diameter of $100 \mathrm{~mm}$ using a pressurized, automated DAD12 system (ALA Scientific Instruments, Farmingdale, NY). For all the studies, no nucleotides or other "regenerating" systems are added to the solutions because recombinant BK activity was found not to run-down under these conditions (Dopico et al. 1996; Dopico 2003; Liu et al. 2006; Liu et al. 2008). All the experiments were performed at room temperature $\left(20^{\circ}-22^{\circ} \mathrm{C}\right)$.

Single channel or macroscopic currents were recorded using an EPC8 amplifier (HEKA, Lambrecht, Germany), low-pass filtered at $1 \mathrm{kHz}$ with an 8-pole Bessel filter (Frequency Devices 902LPF), and digitized at $5 \mathrm{kHz}$ using a Digidata 1320A A/D converter and pCLAMP 8.0 (Molecular Devices). Macroscopic currents were evoked from a holding potential of $-80 \mathrm{mV}$ by 100 milliseconds long, $10 \mathrm{mV}$ depolarizing steps 
ranging from -150 to $+150-200 \mathrm{mV}$. The current amplitude was averaged within $50-100$ milliseconds after the beginning of the depolarizing step.

\subsubsection{Estimating the Number of BK Channels in the Excised Patch}

First, single channel recordings were obtained at very far negative voltages (from $-120 \mathrm{mV}$ to $-20 \mathrm{mV}$ ) at nominal zero $\mathrm{Ca}^{2+}{ }_{\mathrm{i}}$ concentration, estimated $\sim 5 \mathrm{nM}$ (Meera et al. 1996; Cox and Aldrich 2000; Bao and Cox 2005; Orio and Latorre 2005). Then the patch was switched to the solution containing $100 \mu \mathrm{M} \mathrm{Ca}^{2+}{ }_{\text {i }}$ (for cbv1) and $10 \mu \mathrm{M} \mathrm{Ca}^{2+}$ (for cbv $1+\beta 1)$ and thus we recorded macroscopic currents recordings at moderate to high positive voltages $(\sim 30 \mathrm{mV}$ to $200 \mathrm{mV})$. The number of channels $(\mathrm{N})$ in the given excised patch was determined by the Equation 3-1:

$$
N=I /\left(i P_{o}\right)
$$

Where, $I$ represents macroscopic current at a given voltage, and $i$ and $P_{o}$ represent single channel current and open channel probability at the same voltage, respectively.

\subsubsection{Data Analysis}

Patch-clamp data were analyzed with pCLAMP 9.2 (Molecular Devices). From, macroscopic current recording, $\mathrm{G} / \mathrm{G}_{\max }-\mathrm{V}$ curves were fitted by Boltzmann function of the type of Equation 3-2.

$$
G(V)=G_{\max } /\left[1+\exp ^{\left(\frac{V_{0.5}-V}{k}\right)}\right]
$$

Boltzmann fitting routines were run using the Levenberg-Marquardt algorithm to perform nonlinear least square fits. An index of channel activity was obtained from $\mathrm{G} / \mathrm{G}_{\max }-\mathrm{V}$ plots in the form of $\mathrm{V}_{0.5}$ (Bukiya et al. 2009), which is the membrane voltage needed to evoke half-maximal macroscopic current. Smaller $\mathrm{V}_{0.5}$ values indicate increased activity of the channel.

Time constant activation $\left(\tau_{\text {act }}\right)$ and deactivation $\left(\tau_{\text {deact }}\right)$ in milliseconds were obtained by single exponential fits of current activation phase and deactivation time course respectively.

For single channel recordings, the product of number of channels in the patch $(\mathrm{N})$ and channel open probability $\left(\mathrm{P}_{\mathrm{o}}\right)$ was used as an index of channel steady-state activity. Using a built-in function in Clampfit 9.2 (Molecular Devices), $\mathrm{NP}_{\mathrm{o}}$ was obtained from 30 second of gap-free recording under each experimental condition. Data were expressed as mean \pm SEM ( $n=$ number of oocytes/patches), plotted and fitted using Origin 8.5 (Originlab) and InStat3 (GraphPad). 


\subsection{Reversible Permeabilization}

\subsubsection{Solution Composition}

The solution compositions used for reversible permeabilization are as follows. Solution 1 (in mM): 10 EGTA, $120 \mathrm{KCl}, 5 \mathrm{ATP}, 2 \mathrm{MgCl}_{2}, 20 \mathrm{TES}$ (pH 6.8 at $4^{\circ} \mathrm{C}$ ); solution 2 (in $\mathrm{mM}$ ): $120 \mathrm{KCl}, 5 \mathrm{ATP}, 2 \mathrm{MgCl}_{2}, 20 \mathrm{TES}$ (pH 6.8 at $4^{\circ} \mathrm{C}$ ); solution 3 (in $\mathrm{mM}$ ): $120 \mathrm{KCl}, 5 \mathrm{ATP}, 10 \mathrm{MgCl}_{2}, 20 \mathrm{TES}$ (pH 6.8 at $4^{\circ} \mathrm{C}$ ); solution 4 (in $\mathrm{mM}$ ): 140 $\mathrm{NaCl}, 5 \mathrm{KCl}, 10 \mathrm{MgCl}_{2}, 5.6$ glucose, $2 \mathrm{MOPS}$ (pH 7.1 at $22^{\circ} \mathrm{C}$ ) (Lesh et al. 1995; Welsh et al. 2002).

\subsubsection{Principle of Reversible Permeabilization}

The reversible permeabilization technique is based on the principle that when the arteries are placed in solutions 1, 2, and 3 (Lesh et al. 1995; Welsh et al. 2002), ATPbinds to cell surface receptors and removes membrane-associated divalent cations. Thus, by increasing the membrane permeability of smooth muscle cells, selected vectors are delivered inside the myocyte for expression. Later, by removal of extracellular ATP and addition of high $\left[\mathrm{Mg}^{2+}\right]$ in solution 4 reverses the increased membrane permeability followed by graded restoration of physiological concentrations of extracellular $\mathrm{Ca}^{2+}$. Arteries are then placed in DMEM/F-12 culture medium supplemented with penicillin/streptomycin (1\%) for 3-4 days to allow expression of our desired genes. Previous studies demonstrated that reverse permeabilization does not alter the ultrastructural features and contractile properties of smooth muscle cells (Lesh et al. 1995).

\subsubsection{Procedure}

Freshly isolated and de-endothelized mice middle cerebral arteries from $K C N M B 1$ $\mathrm{K} / \mathrm{O}$ mouse were placed into the following four reversible permeabilization solutions sequentially: solution 1 at $4^{\circ} \mathrm{C}$ for 20 minutes, solution 2 containing expression vector $(20 \mu \mathrm{g} / \mathrm{ml})$ at $4^{\circ} \mathrm{C}$ for 90 minutes, solution 3 containing expression vector $(20 \mu \mathrm{g} / \mathrm{ml})$ for 30 minutes at $4^{\circ} \mathrm{C}$, and solution 4 containing expression vector $(20 \mu \mathrm{g} / \mathrm{ml})$ for 30 minutes at room temperature. $\mathrm{Ca}^{2+}$ concentration in solution 4 was then incrementally increased to (in $\mathrm{mM}$ ): $0.01,0.1$ and 1.8 at 15 -minute intervals. Arteries were then placed in DMEM/F12 culture medium containing penicillin/streptomycin (1\%). Then the cultured dishes were incubated in a $74 \% \mathrm{~N}_{2} / 21 \% \mathrm{O}_{2} / 5 \% \mathrm{CO}_{2}$ incubator at $37^{\circ} \mathrm{C}$ for $3-4$ days to allow the expression of gene of interest. After 3-4 days, expression of desired genes in the intact artery was confirmed by Western blotting. 


\subsection{Western Blotting}

\subsubsection{Procedure}

Cerebral arteries were homogenized using RIPA buffer plus protease inhibitor (Thermo Fisher Scientific, Waltham, MA) and whole cell lysate was obtained. Protein samples were heated at $100^{\circ} \mathrm{C}$ for 5 minutes. Protein samples $(30 \mu \mathrm{g} / \mathrm{lane})$ were then separated on a $4-15 \%$ SDS-polyacrylamide gel (Bio-rad, Hercules, CA). To monitor the progress on the gel prestained molecular weight markers were used (Lambda Biotech, SaintLouis, MO). Gel was run in SDS-Tris-Glycine containing MOPS-SDS running buffer at $200 \mathrm{~V}$ for 30 minutes. Following this step, proteins were transferred onto PVDF (polyvinylidene difluoride) membranes using transfer buffer (containing Tris-GlycineMethanol) at $60 \mathrm{~V}$ for 90 minutes. The membranes were then blocked with 5\% non-fat dry milk made in tris-buffered saline (TBS) containing $0.1 \%$ Tween 20 (TBS-T) for 2 hours at room temperature. Then, membranes were incubated with appropriate primary antibodies overnight at $4{ }^{\circ} \mathrm{C}$ in Tris-buffered saline with $0.1 \%$ Tween 20 (TBS-T) and 5\% nonfat dry milk. A $\beta 4$ antibody (1:200 dilution, Alamone) was used to recognize the Nterminus of $\beta 4$ subunit in $\beta_{4} \mathrm{TM} 1_{1}$ and $\beta_{4} \mathrm{TM} 2_{1}$ chimeras; a rabbit polyclonal aniti $\mathrm{BK}-\beta 1$ produced against EC loop of BK- $\beta 1$ subunit (1:500 dilution, Abcam, Cambridge, MA); and mouse monoclonal anti-actin antibody recognizes the epitope located at $\mathrm{N}$-terminus of the actin (1:500 dilution, Milipore, Billerica, MA). Following overnight incubation with primary antibody, membranes were washed six times with TBS-T for 5 minutes each. Membranes were then incubated with appropriate horseradish peroxidaseconjugated secondary antibodies (1:10,000 dilution, Milipore) for 1-2 hours at room temperature. After secondary antibody incubation, membranes were washed with six times with TBS-T for 5 minutes each. Proteins were then visualized using SuperSignal West Pico Chemiluminescent Substrate kit (Thermo Fisher Scientific, Waltham, MA).

\subsubsection{Densitometric Analysis}

Quantity One software v4.6.9 (Bio-Rad, Hercules, CA) was used to calculate the band density. Briefly, rectangular boxes of the same size were placed around bands in each lane, and then the background-subtracted band density was calculated. Average band density was obtained from the same blot acquired at different exposure times. Then, averaged band density for a particular protein sample was normalized to the actin band density of the corresponding sample. 


\subsection{Artery Pressurization and Diameter Measurement}

\subsubsection{Model Justification}

We choose the $K C N M B 1 \mathrm{~K} / \mathrm{O}$ mouse model because: 1) this mouse model has been proven successful in biomedical research to study cell and organism physiology in absence of BK channel $\beta 1$ subunit expression (Xu et al. 1993; Brenner et al. 2000; Brenner et al. 2000; Pluger et al. 2000; Amberg and Santana 2003), including ethanol research studies in our laboratory for several years (Bukiya et al. 2009; Bukiya et al. $2011)$; 2) we have our own colony of $K C N M B 1 \mathrm{~K} / \mathrm{O}$ mice, ensuring availability of animals; 3) we are proficient in determination of cerebral artery diameter and BK function using this model (Bukiya et al. 2009; Bukiya et al. 2011).

Middle cerebral arteries were chosen because: 1) health relevance: middle cerebral artery is the artery most often occluded in stroke (www.strokecenter.org); 2) we conducted ethanol studies using this artery in control $(\mathrm{C} 57 \mathrm{BL} / 6)$ and $K C N M B 1 \mathrm{~K} / \mathrm{O}$ mice (Bukiya et al. 2009; Bukiya et al. 2011).

Adult male 8-12 week-old $K C N M B 1-\mathrm{K} / \mathrm{O}$ and C57BL/6 control mice were decapitated using sharp scissors. From mouse brain, middle cerebral arteries were isolated and cut into $2 \mathrm{~mm}$ long segments on ice under microscope (Nikon SMZ645; Nikon) Sufficient care was taken not to choose arteries with branches or tear in the artery's wall. Endothelium was removed by passing an air bubble into the vessel lumen for 90 seconds. Endothelium-denuded artery was cannulated at each end to the temperature-controlled perfusion chamber (Living Systems Instrumentation, Burlington, VT) containing PSS. Artery cannulated-chamber was mounted on the microscope (Nikon TS100). A Dynamax RP-1 peristalatic pump (Rainin Instruments, Inc., Oakland, CA) was used to perfuse the Physiological Saline Solution (PSS) (mM): $119 \mathrm{NaCl}, 4.7 \mathrm{KCl}, 1.2$ $\mathrm{KH}_{2} \mathrm{PO}_{4}, 1.6 \mathrm{CaCl}_{2}, 1.2 \mathrm{MgSO}_{4}, 0.023$ EDTA, 11 glucose, and $24 \mathrm{NaHCO}_{3}$. PSS was equilibrated at $\mathrm{pH} 7.4$ with a $21 / 5 / 74 \%$ mix of $\mathrm{O}_{2} / \mathrm{CO}_{2} / \mathrm{N}_{2}$ and maintained at $35-37^{\circ} \mathrm{C}$. Intravascular pressure was applied using water filled reservoir and pressure was monitored using a pressure transducer (Living Systems Instrumentation, Burlington, VT). A CCD camera (Sanyo VCB-3512T; Sanyo Electric Co., Moriguchi, Japan) was attached to an inverted microscope (Nikon Eclipse TS100; Nikon) to monitor changes in artery diameter. The external wall diameter of the artery was measured at $1 \mathrm{kHz}$ in real time using the automatic edge-detection function of IonWizard software (IonOptics, Milton, MA).

\subsubsection{Procedure}

At the onset of the experiment, arteries were incubated at an intravascular pressure of $10 \mathrm{mmHg}$ for 10 minutes. After the arteries attained the stable diameter under this condition, intravascular pressure was increased to $\sim 60 \mathrm{~mm} \mathrm{Hg}$. This pressure was maintained throughout the experiment. The increased intravascular pressure resulted in 
arterial dilation. The artery was then allowed to sense the elevated intravascular pressure and develop pressure-induced arterial constriction over a period of time (equivalent to myogenic tone). Once myogenic tone remained steady for a period of 15-30 minutes, pharmacological agents were perfused. Drugs were dissolved from stock solutions into PSS to achieve their final concentration and applied via perfusion chamber containing artery. After drug perfusion, the effect the particular drug was evaluated at the time when it evoked a steady diameter value, whether reduction (constriction) or enhancement (dilation).

After pressurization, first, artery contractility was tested with $60 \mathrm{mM} \mathrm{K}^{+}$, which shifts the myocyte membrane potential from -40 to $-20 \mathrm{mV}$ and produces maximal contraction (Liu et al., 2004). After washout, bath application of $50 \mathrm{mM}$ ethanol for 10 minutes in PSS was performed. After ethanol washout, $45 \mu \mathrm{M}$ Lithocholate (LCA) was perfused. The maximal change in diameter reached within 10 minutes from the start of drug application was considered as the dependent variable. At the end of the experiment, passive diameter of the vessel was determined in the $\mathrm{Ca}^{2+}$-free PSS (no $\mathrm{CaCl}_{2}$ added, plus $10 \mathrm{mM}$ EGTA to chelate trace amounts $\mathrm{Ca}^{2+}$ ) (Bukiya et al. 2007; Bukiya et al. 2011). Arteries that failed to constrict for $\mathrm{KCl}$ and/or dilate in response $\mathrm{Ca}^{2+}$-free PSS were discarded from analysis. The absence of a functional endothelium was determined with an application of the endothelium-dependent vasodilator acetylcholine $(10 \mu \mathrm{M}) v s$. the endothelium-independent vasodilator sodium nitroprusside (NPS) (Liu et al. 2004; Bukiya et al. 2007).

\subsubsection{Analysis}

Ion Wizard software was used to measure artery diameter traces. Diameter measurement was obtained when artery attained stable diameter before and after application of each drug. Myogenic tone (in \%) was determined using the Equation 3-3:

$$
\% \text { Change in Diameter }=\left[1-\left(\frac{\text { Active diameter }}{\text { Passive diameter }}\right)\right] X 100
$$

\subsection{Chemicals}

$100 \%$ pure ethanol was purchased from American Bioanalytical (Natick, MA). Lithocholate (LCA; $5 \beta$-cholanic acid-3 $\alpha$-ol) was purchased from Steraloids (Newport, RI). All other chemicals were purchased from Sigma Aldrich. Just before the experiment, LCA was initially dissolved in DMSO to obtain stock solution of $333 \mathrm{mM}$. Stock solution was sonicated for 30 minutes. Then, to obtain final concentration, stock solution was further diluted with PSS solution. As a control for LCA experiments, DMSO-containing PSS solution was perfused. Concentration of DMSO in "control" experiment matched the corresponding amount of DMSO in LCA-containing solution. 


\subsection{Statistical Analysis}

Data were plotted and fitted using OriginLab 8.5. Statistical analysis was performed using GraphPad InStat software. In order to compare paired or unpaired data from two populations, Student's t-test with two-tail $\mathrm{p}$ value was used. On the other hand, ordinary one-way ANOVA followed by multiple Bonferonni test was used for all multiple data comparisons. $\mathrm{p}<0.05$, was considered statistical significance. Values were expressed as mean \pm SEM., and $n=$ number of patches/arteries. Each patch was obtained from a different oocyte, and each pressurized artery measurement was obtained from a middle cerebral artery segment dissected out from a different animal. 


\section{CHAPTER 4. THE CHANNEL'S ACTIVATING ION DETERMINES ETHANOL MODULATION OF BK CHANNELS}

\subsection{Overview}

Ethanol levels reached in circulation during moderate to heavy alcohol intoxication (30-60 $\mathrm{mM}$ ) as during binge drinking modify BK channel steady-state activity $\left(\mathrm{NP}_{\mathrm{o}}\right)$ and thus, BK channel-dependent physiology and behavior. In general, ethanol increases $\mathrm{NP}_{\mathrm{o}}$ of neuronal $\mathrm{BK}$ channels while decreasing $\mathrm{NP}_{\mathrm{o}}$ of vascular smooth muscle BK channels. There are various factors that play a role in ethanol's final effect on $\mathrm{BK} \mathrm{NP}_{\mathrm{o}}$, including, channel subunit composition, post-translational modification of $\mathrm{BK}$ subunits, and the channel lipid microenvironment (Brodie et al. 2007). However, data from reductionist systems, such artificial bilayers of simple lipid composition where BK channel-forming (slo1) subunits can be functionally evaluated, reveal that ethanol modulation of $\mathrm{BK} \mathrm{NP}$ olely requires slo1 subunits and the channel's physiological activator, that is $\mathrm{Ca}^{2+}$ i (Crowley et al. 2003; Crowley et al. 2005; Liu et al. 2008). Moreover, ethanol action on slo1 $\mathrm{NP}_{\mathrm{o}}$ is a function of activating $\left[\mathrm{Ca}^{2+}\right]_{\mathrm{i}}$ (Liu et al. 2008; Bukiya et al. 2009).

Slo2.1 (Slick), Slo2.2 (Slack), and Slo3 gene products render ion channel proteins that display a phenotype common to slo1: high conductance for $\mathrm{K}^{+}$and dual regulation of gating by transmembrane voltage and intracellular ion-recognition, with slo2 and slo3 being activated by $\mathrm{Na}^{+}{ }_{\mathrm{i}}$ and $\mathrm{OH}^{-}$, respectively (Salkoff et al. 2006). Whether the control of ethanol action on BK channels by activating ion is unique to slo1 or, rather, extends to the other members of the slo family remains unknown. To address this question, I expressed BK channel pore-forming $\alpha$ subunits cloned from rat cerebral artery myocytes (cbv1) in Xenopus laevis oocytes. The cbv1 channel variant showed all properties of other slo1 channels, such as hslo1 (Brenner et al. 2000; Cox and Aldrich 2000), mslo1 (Liu et al. 2008; Liu et al. 2013) including independent gating by $\mathrm{Ca}^{2+}{ }_{\mathrm{i}}$ and $\mathrm{Mg}^{2+}{ }_{\mathrm{i}}$. Using the same expression system and the inside-out configuration of the patch-clamp technique, which allows tight control of activation ion concentrations by perfusing with the cytosolic side of the channel (where the ion-sensors are located). I also expressed slo2 and slo3 and thus, I probed cbv1, slo2 and slo3 channels with $50 \mathrm{mM}$ ethanol over a wide range of channel activating ion concentration. Then, I constructed conductance $(\mathrm{G})$ or $\mathrm{NP}_{\mathrm{o}}$-voltage (V) plots from macroscopic current amplitude or $\mathrm{P}_{0}$, depending on the level of channel expression. This study tests the following hypotheses:

- Slo1 channel sensitivity to ethanol is selectively dependent on its activating ion $\mathrm{Ca}^{2+}$ i.

- Ethanol differentially modulates the different members of the slo channel family i.e. slo1, slo2 and slo3 


\section{2. $\quad$ Results}

\subsubsection{Ethanol Modulation of Slo1 Channel's Activity Is Selectively Dependent on Its Activating Ion i.e., $\mathrm{Ca}^{2+}{ }_{i}$}

In this study, macroscopic current recordings were obtained from excised insideout patches over a wide of range activating $\mathrm{Ca}^{2+}{ }_{i}$ concentration $(0-100 \mu \mathrm{M})$ in presence and absence of $50 \mathrm{mM}$ ethanol. Iso-osmotically ethanol-substituting urea in bath solution was used as control. Voltage protocols were identical to those previously described, (see methods section 3.4.1). From G-V plots, we obtained the voltage required to achieve half of the maximal conductance/ $\mathrm{NP}_{\mathrm{o}}\left(\mathrm{V}_{0.5}\right)$. Data demonstrate that ethanol activates cbv1 channel activity at low $\left[\mathrm{Ca}^{2+}\right]$ i.e., $0.3-10 \mu \mathrm{M}$, which is evident as a shift to the left in $\mathrm{G} / \mathrm{G}_{\max }-\mathrm{V}$ plots and significant decreases in $\mathrm{V}_{0.5}$ values in the $\mathrm{V}_{0.5}-\left[\mathrm{Ca}^{2+}\right]$ plot

(Figure 4-1A and B, Figure 4-2A and B). First, ethanol did not modulate cbv1 channel activity at zero $\mathrm{Ca}^{2+}{ }_{i}$ (Figure 4-1D and Figure 4-2B). Second, as $\left[\mathrm{Ca}^{2+}\right]$ increases, ethanol-induced channels activation turns to mild inhibition, which is evident as small right-shift in the $\mathrm{G} / \mathrm{G}_{\max }-\mathrm{V}$ plot and increase in $\mathrm{V}_{0.5}$. Ethanol-induced activation-toinhibition cross-over occurs at $\sim 20 \mu \mathrm{M}$ (Figure 4-1A and C, Figure 4-2A and B). Thus, ethanol action on cbv1 channels not only is dependent on $\mathrm{Ca}^{2+}{ }_{i}$ presence but is a function of $\left[\mathrm{Ca}^{2+}\right]_{i}$, which extends previous findings with mslol channels in the same expression system (Liu et al. 2008).

\subsection{2. $\mathrm{Mg}^{2+}-/$ Voltage-Gated Cbv1 Channels Are Ethanol Insensitive}

Next we decided to test the possibility that whether ethanol modulates cbv1 channels-gated independently of its physiological activating ion i.e., $\mathrm{Ca}^{2+}{ }_{\text {i. }}$ Previous studies have clearly established that BK channels are gated by $\mathrm{Mg}^{2+}$ and voltage, independently of $\mathrm{Ca}^{2+}{ }_{\mathrm{i}}$ (Shi et al. 2002). Thus, next we explored ethanol action on $\mathrm{Mg}^{2+} / \mathrm{V}$-gated cbv1 channels in similar experimental conditions to those of cbv1 channel.

Before probing the channels with ethanol, functional expression and characteristic phenotype (such as voltage and magnesium sensitivity) of $\mathrm{Mg}^{2+} / \mathrm{V}$-gated cbv1 channels was verified. In this particular set of experiments, it should be underscored that the electrode solution did not have any added calcium (see section 3.4.1). As their characteristic feature, $\mathrm{Mg}^{2+} / \mathrm{V}$-gated cbv1 channels, in presence of increasing concentration of $\mathrm{Mg}^{2+}$ on intracellular side of the channel, the activity of channels was increased monotonically. This increase in activity was evident as a decrease in $\mathrm{V}_{0.5}$ value as $\mathrm{Mg}^{2+}$ concentration was increased from $1 \mathrm{mM}$ thru $100 \mathrm{mM}$. These results are in agreement with previously reported studies (see control data of Figure 4-3A, B, C and D) (Shi et al. 2002). Then, we explored ethanol action on $\mathrm{Mg}^{2+} / \mathrm{V}$-gated cbv1 channels. Here, we obtained macroscopic current recordings over a wide range of activating magnesium concentration $(0-100 \mathrm{mM})$ in presence and absence of $50 \mathrm{mM}$ ethanol (Figure 4-3A). 

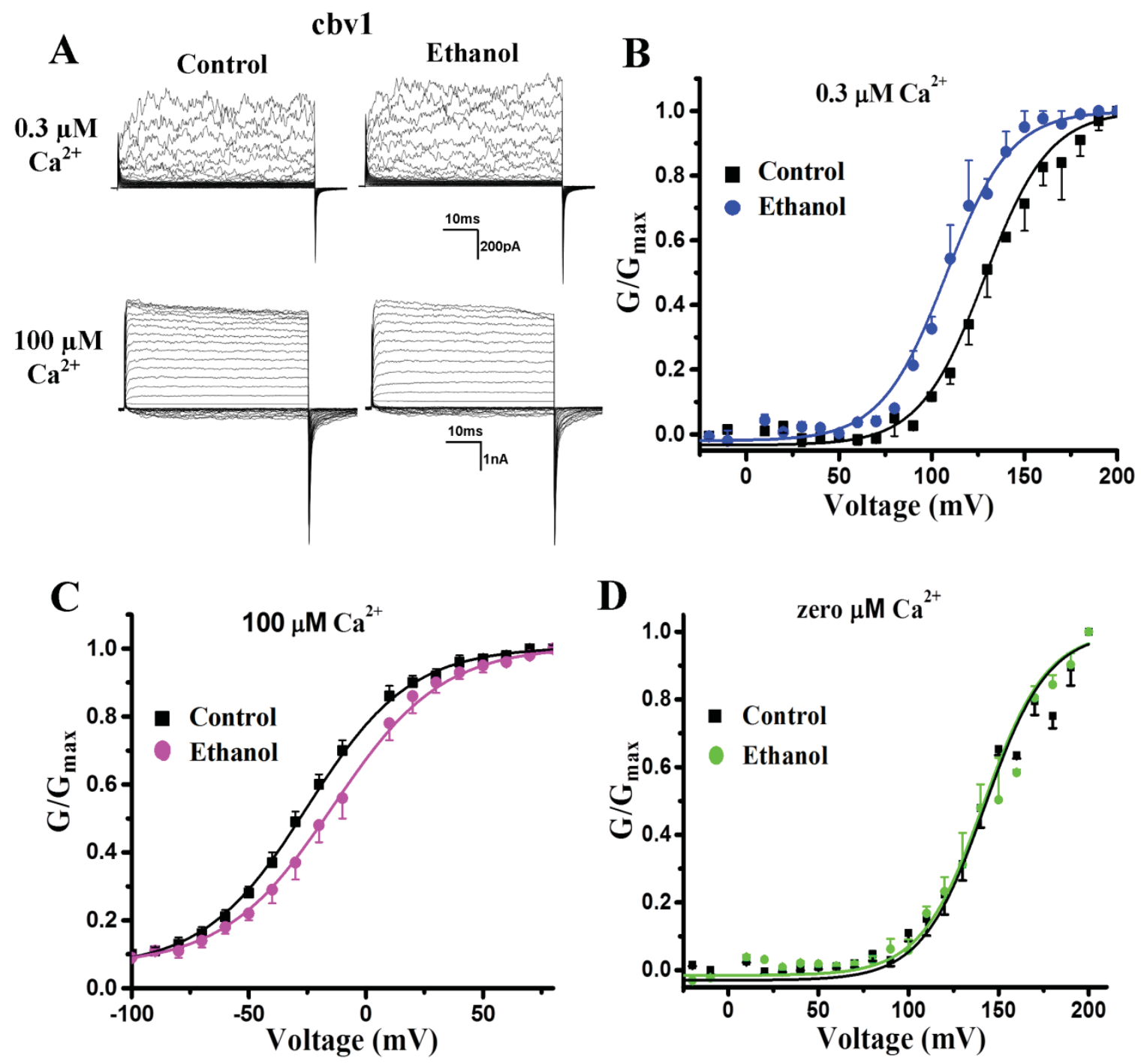

Figure 4-1. Ethanol effect on BK channel activity is $\mathrm{Ca}^{2+}{ }^{2}$-dependent

Notes: Ethanol $(50 \mathrm{mM})$ activates homomeric cbv1 channels at submicromolar $(0.3 \mu \mathrm{M})$ $\mathrm{Ca}^{2+}{ }_{i}$ while causing mild inhibition at higher $\mathrm{Ca}^{2+}{ }_{i}(100 \mu \mathrm{M})$. A) Macroscopic current recordings evoked from I/O patches at 0.3 and $100 \mu \mathrm{M} \mathrm{Ca}^{2+}{ }_{i}$ in absence or presence of 50 $\mathrm{mM}$ ethanol following cbv1 expression in Xenopus oocytes. B) At $0.3 \mu \mathrm{M} \mathrm{Ca}^{2+}{ }_{\mathrm{i}}$, ethanol shifts the $\mathrm{G} / \mathrm{G}_{\max }-\mathrm{V}$ plot to the left indicating channel activation. C) At $100 \mu \mathrm{M} \mathrm{Ca}^{2+}{ }_{i}$ ethanol shifts the $G / G_{\max }-V$ plot to the right indicating inhibition of channel activity. D) At nominal zero $\mu \mathrm{M} \mathrm{Ca}^{2+}{ }_{i}$ ethanol did not shift the $\mathrm{G} / \mathrm{G}_{\max }-\mathrm{V}$ plot indicating ethanol fails to modulate cbv1 channel activity at nominal zero $\mathrm{Ca}^{2+}{ }_{\mathrm{i}}$. 

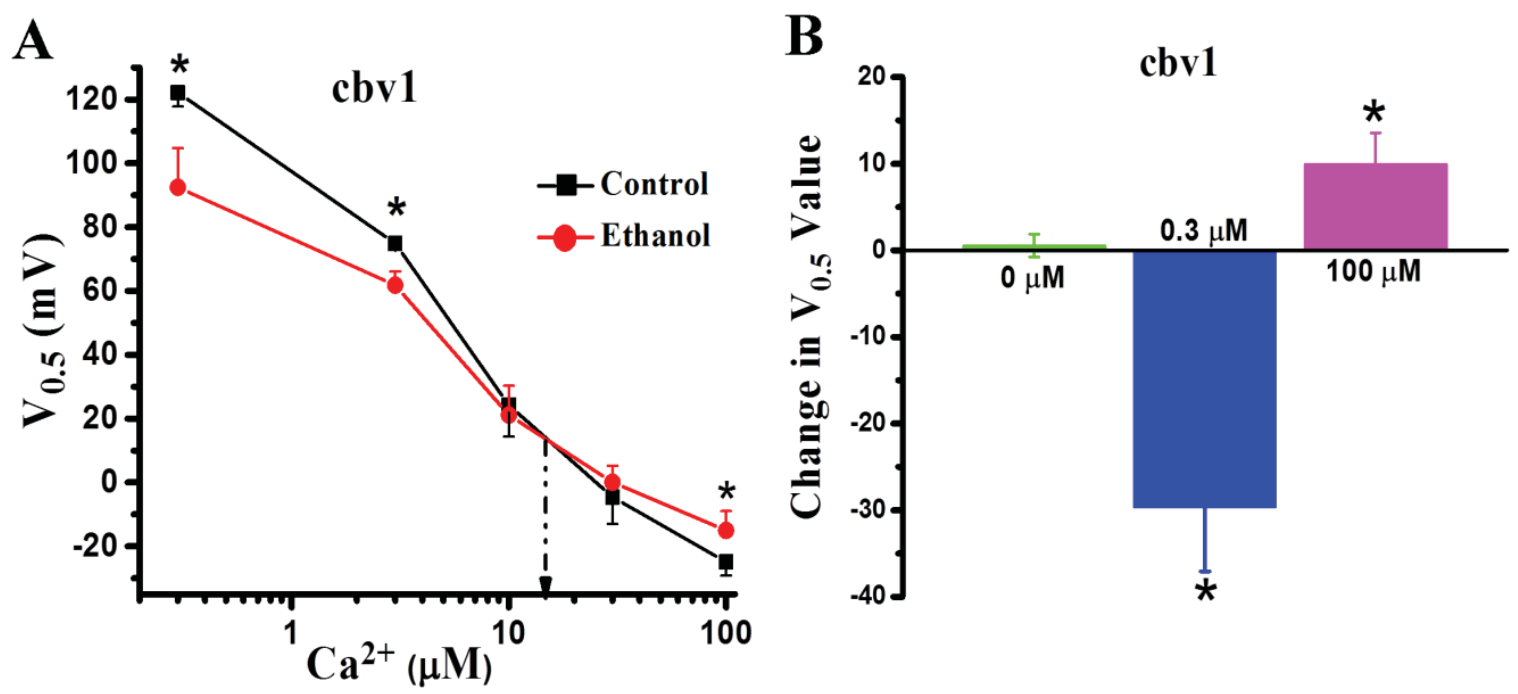

Figure 4-2. Ethanol action on $\mathrm{BK}$ channel activity is function of $\mathrm{Ca}^{2+}{ }_{\mathrm{i}}$

Notes: Ethanol activates homomeric cbv1 channels at submicromolar $\mathrm{Ca}^{2+}{ }_{i}$ while causing mild inhibition at higher $\mathrm{Ca}^{2+}{ }_{\mathrm{i}}$. A) $\mathrm{V}_{0.5} v s$. $\left[\mathrm{Ca}^{2+}{ }_{\mathrm{i}}\right]$ plot showing that the activation-toinhibition crossover for ethanol effect on cbv1 channels occurs at $\approx 20 \mu \mathrm{M} \mathrm{Ca}^{2+}{ }_{\mathrm{i}} \mathrm{B}$ ) Bar graph showing ethanol-induced change in $\mathrm{V}_{0.5}$ values from control obtained at $0.3 \mu \mathrm{M}$ and $100 \mu \mathrm{M} \mathrm{Ca}^{2+}{ }_{i}$. Each data point represents the average of no less than 3 patches, each excised from a different cell. *Different from control $(\mathrm{p}<0.05)$. 


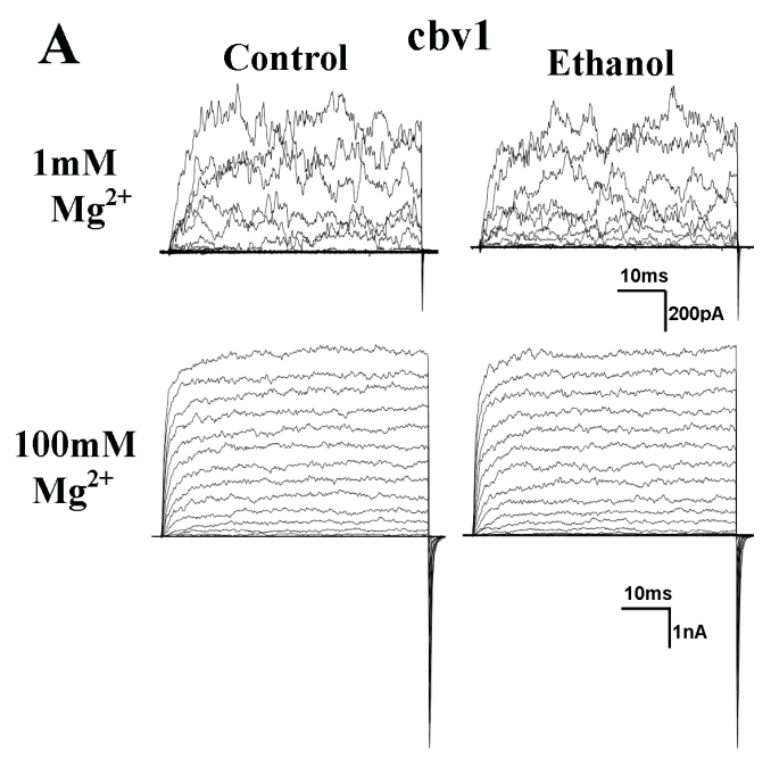

B
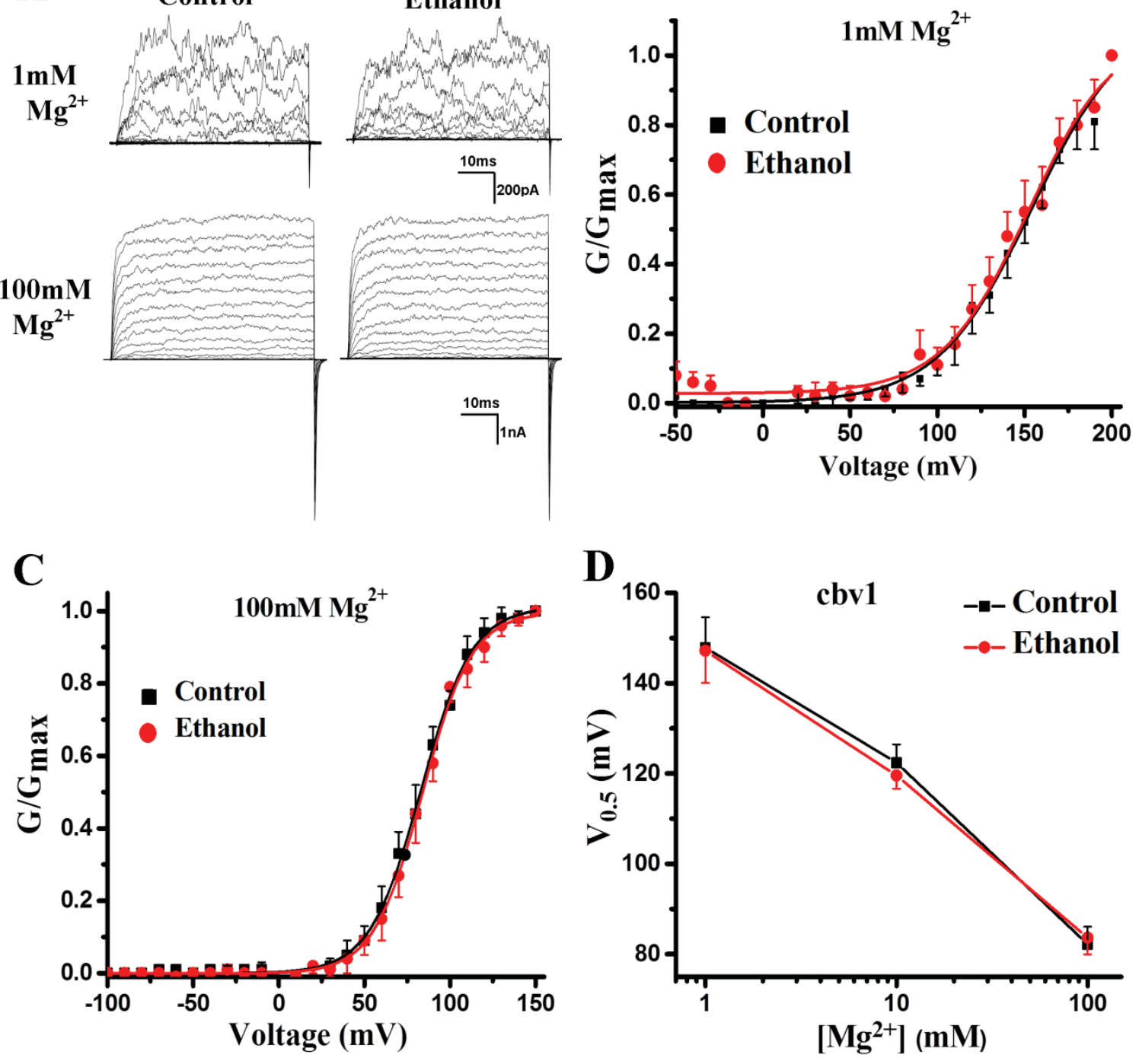

Figure 4-3. $\mathrm{Mg}^{2+} /$ voltage-gated cbv1 channels are ethanol-insensitive

Notes: In absence of $\mathrm{Ca}^{2+}$, ethanol $(50 \mathrm{mM})$ does not modulate cbv1 channel activity whether evoked at 1,10 or $100 \mathrm{mM} \mathrm{Mg}^{2+}{ }_{\mathrm{i}}$. A) Macroscopic current recordings from $\mathrm{I} / \mathrm{O}$ patches recorded at 1 and $100 \mathrm{mM} \mathrm{Mg}^{2+}{ }_{\mathrm{i}}$ in absence or presence of $50 \mathrm{mM}$ ethanol following cbv1 expression in Xenopus oocytes. B, C) In absence of $\mathrm{Ca}^{2+}$, ethanol does not shift the $\mathrm{G} / \mathrm{G}_{\max }-\mathrm{V}$ plots whether obtained at physiological (1 $\mathrm{mM}$ ) or supraphysiological $(100 \mathrm{mM}) \mathrm{Mg}^{2+}$ i. D) $\mathrm{V}_{0.5} \mathrm{vs}$. $\left[\mathrm{Mg}^{2+} \mathrm{i}\right]$ plot showing the absence of ethanol effect on cbv1 channels over the wide range of $\left[\mathrm{Mg}^{2+}\right]_{\mathrm{i}}$ chosen. 
Our data show that ethanol failed to modulate the BK channel activity over a wide range of activating $\left[\mathrm{Mg}^{2+}\right]$, which is evident from the lack of significant differences in $\mathrm{G} / \mathrm{G}_{\max }-\mathrm{V}$ and $\mathrm{V}_{0.5}-\left[\mathrm{Mg}^{2+}\right]$ plots between control and ethanol-containing solution

(Figure 4-3B, C and D). Collectively, these findings suggest that when cbv1 channels are gated by $\mathrm{Mg}^{2+} /$ Voltage, their ethanol-sensitivity is lost.

\subsubsection{Slo2 Channels Are Ethanol Insensitive over a Wide Range of Their Activating Ion Concentration}

We next aimed to address whether ethanol distinctly modulates slo1 channels or, rather, it can also regulate slo2 and slo3 channel activity

First, we evaluated the ethanol action on slo2 channels, which are phylogenetically related to slo1 channels (Santi et al., 2006). Slo2 channels are predominantly expressed in neurons and the heart, thus participate in adaptation of neuronal firing rate and slow afterhyperpolarizations that follow repetitive firing (Bhattacharjee and Kaczmarek 2005; Salkoff et al. 2006) and protection of excitable cells against hypoxia (Dryer 1994). Like slo1 channels, mammalian slo2 channels, exhibit high conductance for $\mathrm{K}^{+}$ions. However, they are sensitive to $\mathrm{Na}^{+}{ }_{\mathrm{i}}$ and $\mathrm{Cl}^{-}{ }_{\mathrm{i}}$ while being insensitive to physiologic levels of $\mathrm{Ca}^{2+}$ i. (Bhattacharjee and Kaczmarek 2005; Salkoff et al. 2006).

From a structural point of view, slo2 channels are made of an intracellular Nterminus, TM6 transmembrane core (S1-S6) and intracellular cytosolic tail domain that contains the RCKs. The latter are involved in sensing of $\mathrm{Na}^{2+}$ and $\mathrm{Cl}^{-}$ions. However, these channels are largely $\mathrm{Ca}^{2+}$-insensitive. Noteworthy, in contrast to slo 1 channels, slo2 channels do not possess S0 TM domain and S0-S1 cytosolic loop (Liu et al. 2013) (Figure 4-4A).

In this study, we used a combination of rslo2.1 and rslo2.2 cRNAs at a 1:1 molar ratio, which results in heteromeric rslo2.1:rslo2.2, $\mathrm{Na}^{+}$-gated $\mathrm{K}^{+}$channels (Salkoff et al. 2006; Chen et al. 2009; Zhang et al. 2010). In the presence of intracellular activating $\mathrm{Na}^{+}$i (i.e., $70 \mathrm{mM} \mathrm{Na}{ }^{+}$gluconate in the bath solution), we routinely obtained single channel recordings with an averaged unitary current amplitude of $6.1 \pm 0.5 \mathrm{pA}$ at +50 $\mathrm{mV}$, as previously observed from slo2 channels permeating $\mathrm{K}^{+}$(Santi et al. 2006). As typically expected from these channels, rslo2.1:rslo2.2 channel activity was monotonically increased within a wide concentration of $\mathrm{Na}^{+}(10-140 \mathrm{mM})$

(Figure 4-4B and C) while being unresponsive to physiological levels of intracellular $\mathrm{Ca}^{2+}$. Further, unlike slo1 channels, slo2 channels are proven to be very less voltage sensitive, (Santi et al. 2006)therefore, we obtained single channel recordings for studies involving slo2 channels.

Next, we investigated whether modification of activating $\mathrm{Na}^{+}{ }_{\mathrm{i}}$ levels could render rslo2.1:rslo2.2 channels sensitive to ethanol. At all $\mathrm{Na}^{+}{ }_{\mathrm{i}}$ levels tested $(10-140 \mathrm{mM})$, the channel remained consistently resistant to $50 \mathrm{mM}$ ethanol (Figure 4-4B and C). 


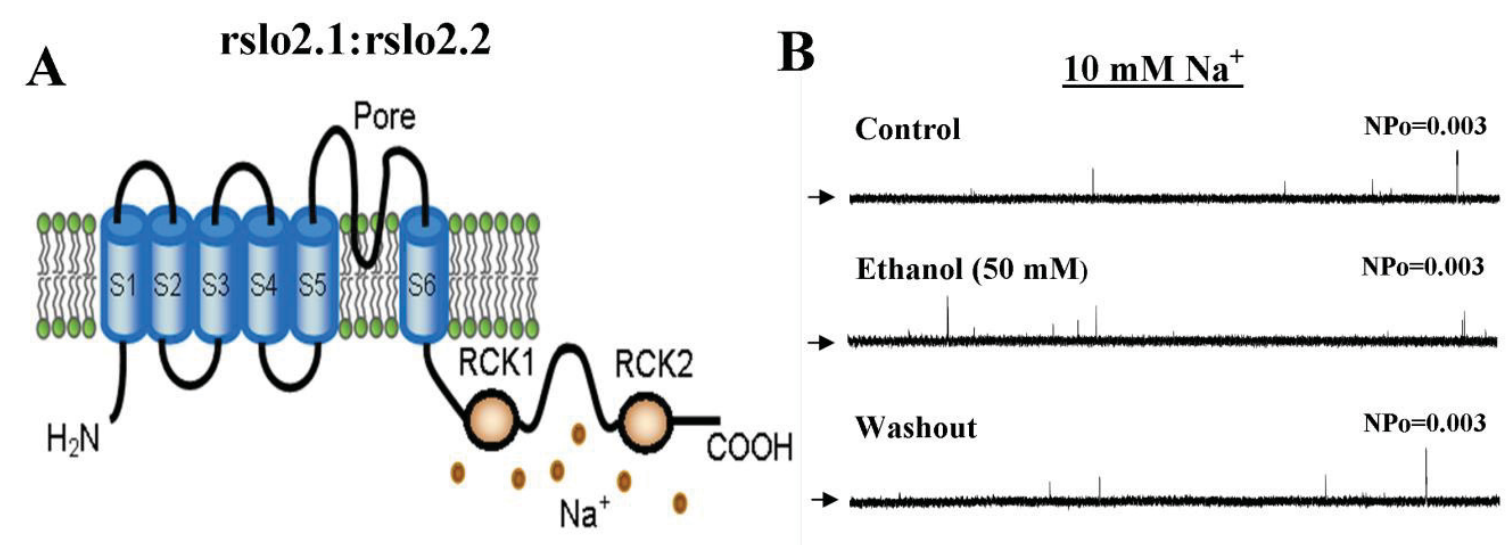

C
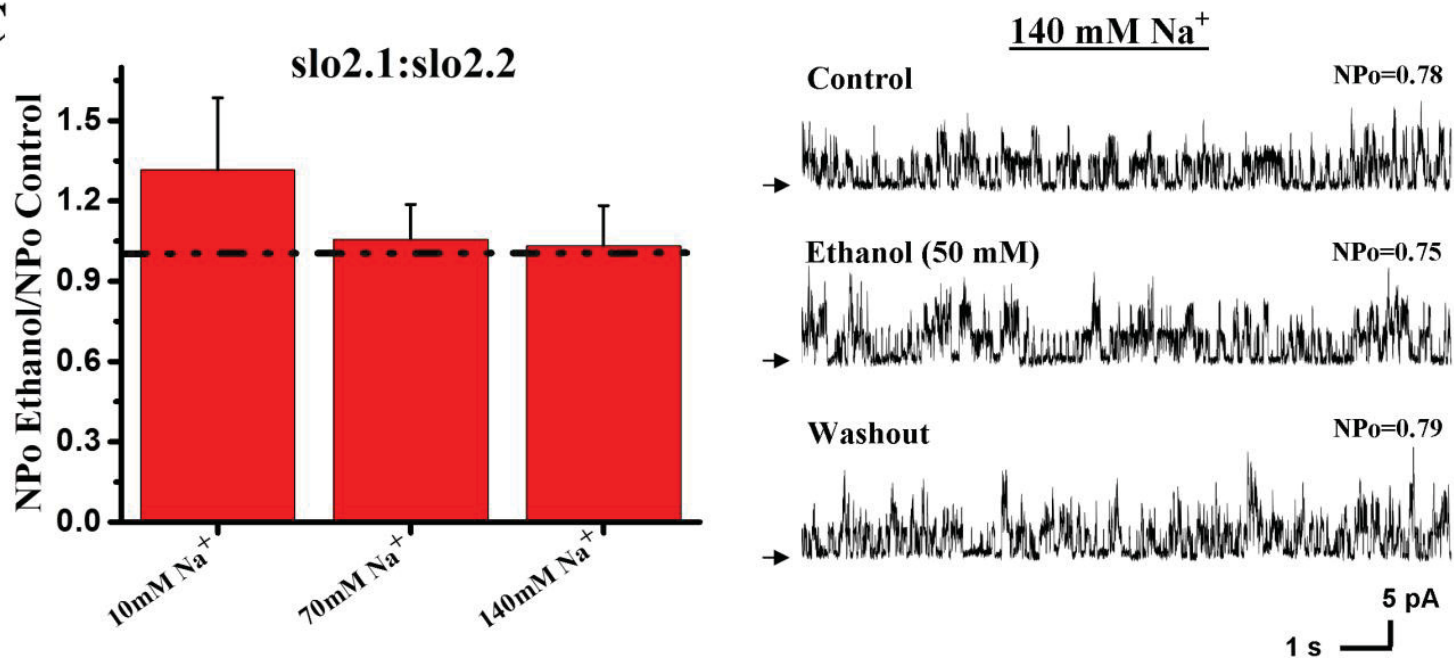

Figure 4-4. Slo2.1:slo2.2 channels are insensitive to ethanol at a wide range of activating $\mathrm{Na}^{+}{ }_{i}$

Notes: A) Cartoon depicting a rslo2 monomer inserted in the membrane, showing slo2 TM segments and functional domains. B) Single channel recordings of slo2.1:slo2.2 channels recorded from I/O patches at 10 (top panel) or $140 \mathrm{mM}$ (bottom panel) $\mathrm{Na}^{+}{ }_{\mathrm{i}}$; $\mathrm{V}_{\mathrm{m}}=+90 \mathrm{mV}$. Records were obtained before (top trace on each panel), during (middle trace), and immediately after (bottom trace) patch exposure to $50 \mathrm{mM} \mathrm{EtOH}$. Arrows indicate the baseline (all channels closed). C) Averaged $\mathrm{NP}_{\mathrm{o}}$ ratios in presence and absence of ethanol from slo2.1:slo2.2 at $10(n=3), 70(n=4)$ and $140 \mathrm{mM} \mathrm{Na}^{+}{ }_{\mathrm{i}}(\mathrm{n}=4)$. In contrast to $\mathrm{Ca}^{2+}{ }_{i}$ and slo1, the presence of $\mathrm{Na}^{+}{ }_{i}$ cannot render slo2 channels ethanolsensitive. A dotted line underscores lack of ethanol action.

Source: Modified with permission from Liu, J., Bukiya, A.N., Kuntamallappanavar, G., Singh, A.K. and Dopico, A.M. (2013). Disntict sensitivity of Slo1 channel proteins to ethanol. Mol. Pharmacol 83(1): 235-244. 
This resistance of slo2 channels to ethanol is being in sharp contrast with the ethanol response from slo1 channels. Noteworthy, slo2 refractoriness to $50 \mathrm{mM}$ ethanol is found over much wider range (considering both actual concentration and modification in channel activity) of $\mathrm{Na}^{2+}$ than $\mathrm{Ca}^{2+}{ }_{i}$ that determines different slo1 responses to ethanol as follows: activation, refractoriness, and inhibition (Liu et al. 2008; Bukiya et al. 2009). Therefore, rslo2.1:rslo2.2 channels remain insensitive to intoxicating concentrations of ethanol even across a wide range of activating ion concentration and eventual changes in channel activity.

\subsubsection{Slo3 Channels Are Ethanol Insensitive over a Wide Range of Their Activating Ion Concentration}

We next addressed whether slo3 channels responded to ethanol like slo1 or, rather, remain resistant as found for slo2 (see previous section).

The $\mathrm{H}^{+}$sensitive $\left(\mathrm{OH}^{-}\right.$-activated) slo3 channels are predominantly found only in mammalian testis, which contrasts with the wide distribution of slo1 and slo2 channels (Salkoff et al. 2006). Structurally, slo3 channels contain an N-terminus, seven transmembrane domains (S0-S6) and intracellular CTD-containing RCKs, which distinctly sense $\mathrm{OH}^{-}$ions (Figure 4-5A). It should be underscored that, in contrast to slo1 channels, RCKs of slo3 channels do not sense physiological levels of $\mathrm{Ca}^{2+}$ (Salkoff et al. 2006).

Before investigating the ethanol action on slo3 channels, their functional expression in oocytes was confirmed. In the presence of intracellular activating alkaline medium (bath solution $\mathrm{pH}=8.0,\left[\mathrm{OH}^{-}\right]=1 \mu \mathrm{M}$ ), we were able to obtain single-channel events with unitary current amplitudes of $2.4 \pm 0.3 \mathrm{pA}$ at $+80 \mathrm{mV}$, as previously reported for slo3 channels (Schreiber et al. 1998; Qian et al. 2002; Xia et al. 2004). Further, slo3 channel activity was consistently increased as $\mathrm{OH}^{-}$i concentration was increased from $0.01 \mu \mathrm{M}$ thru $10 \mu \mathrm{M}$ (which is equivalent to $\mathrm{pH}=6.0$ thru $\mathrm{pH}=9.0$ ) (Figure 4-5B and $\mathbf{C}$ ). These results are in similar to previously published reports (Xia et al. 2004). Further, expression levels of slo3 channels were relatively low compared to slo1 channels. It is evident by brief channel openings in Figure 4-5B. Thus, macroscopic currents resulting from slo3 channels were of low magnitude and did not fit Boltzmann very well.

Then, $50 \mathrm{mM}$ ethanol was applied to the cytosolic side of membrane patches to determine the ethanol-sensitivity of slo3 channels. Since the ethanol action on slo1 channel activity was a function of $\left[\mathrm{Ca}^{2+}\right]_{\mathrm{i}}$, we conducted this study at a wide range of activating levels of $\mathrm{OH}^{-}$. Our results, however, demonstrate that at all $\mathrm{OH}_{\mathrm{i}}^{-}$levels tested $(0.01-10 \mu \mathrm{M})$, the slo3 channel remained consistently unresponsive to $50 \mathrm{mM}$ ethanol while being sensitive to $\mathrm{OH}_{i}^{-}$levels (Figure 4-5B and $\mathbf{C}$ ), a result that contrasts with that of slo1 channels while paralleling findings from slo2. 


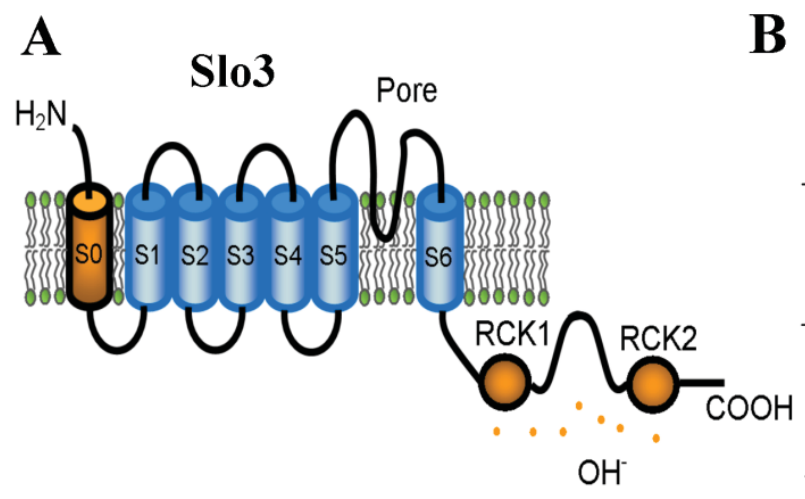

B
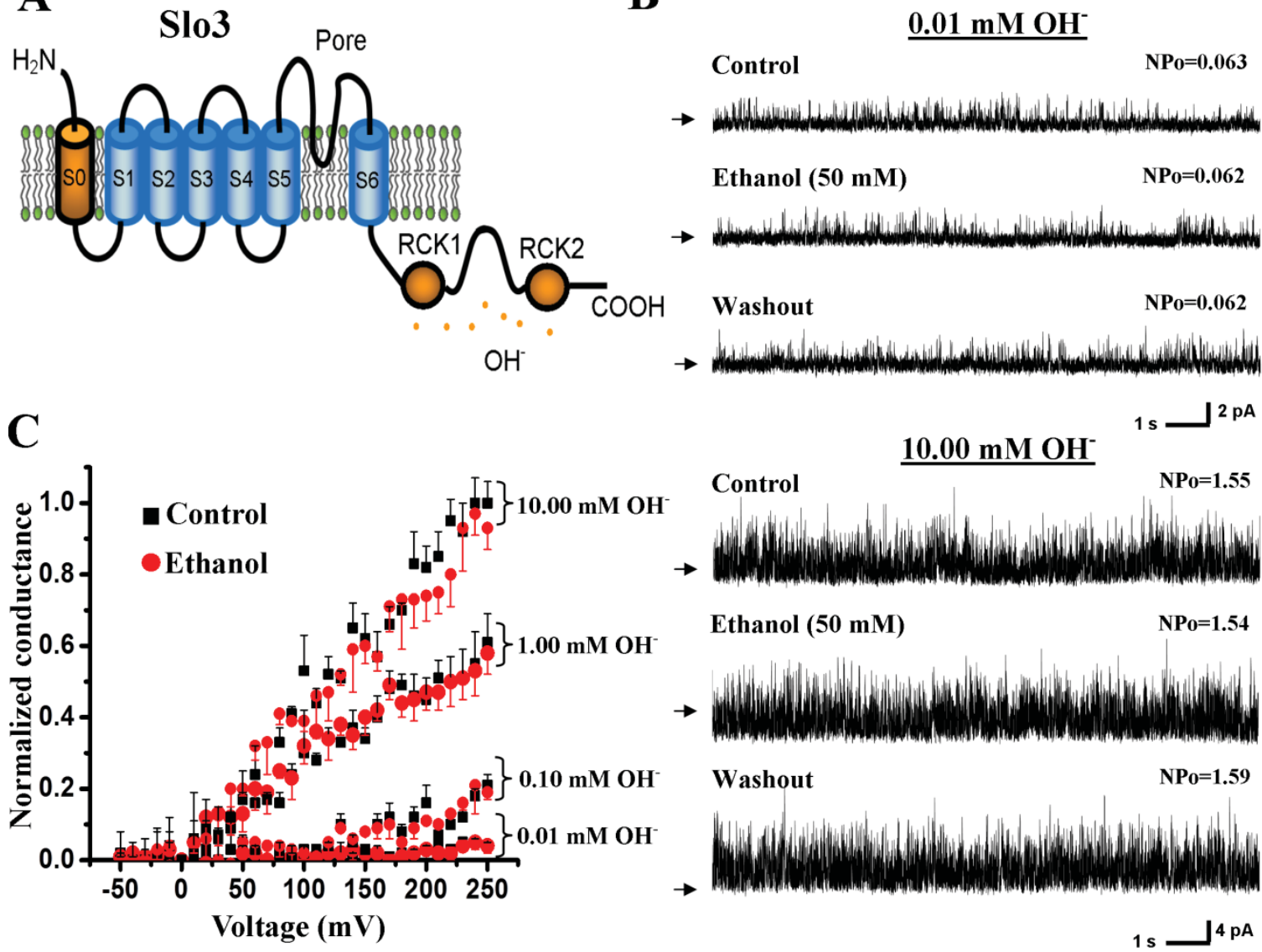

Figure 4-5. Slo3 channels are ethanol-insensitive at a wide range of activating $\mathrm{OH}^{-}$

Notes: A) Cartoon depicting a slo3 monomer inserted in the membrane, showing slo3 TM segments and functional domains. B) Single-channel recordings of slo3 channels from $\mathrm{I} / \mathrm{O}$ patches at 0.01 (top panel) and $10 \mu \mathrm{M} \mathrm{OH}^{-}$(bottom panel); $\mathrm{V}_{\mathrm{m}}=+60 \mathrm{mV}$. Records were obtained before (top trace on each panel), during (middle trace), and immediately after (bottom trace) patch exposure to $50 \mathrm{mM} \mathrm{EtOH}$. Arrows indicate the baseline (all channels closed). C) slo3 averaged macroscopic conductances (G) at 0.01, 0.1, 1 and 10 $\mu \mathrm{M} \mathrm{OH}_{\mathrm{i}}^{-}$(normalized to $\mathrm{G}_{\max }$ obtained at $10 \mu \mathrm{M} \mathrm{OH}^{-}$) in presence and absence of ethanol. Each data point represents the average of no less than 3 patches; each patch was excised from a different cell.

Source: Modified with permission from Liu, J., Bukiya, A.N., Kuntamallappanavar, G., Singh, A.K. and Dopico, A.M. (2013). Disntict sensitivity of Slo1 channel proteins to ethanol. Mol. Pharmacol 83(1): 235-244. 


\subsection{Discussion}

In this study, we tried to address the questions, 1) whether ethanol action on slo1 channels was selectively dependent on its activating ion i.e., $\mathrm{Ca}^{2+}{ }_{i}$ and 2) ethanol sensitivity is unique to slo1 (cbv1) channels or it also extends to other members of slo family such as slo2, and slo3 channels. Our investigation was carried out in same expression system, under similar patch-clamp recording conditions and across wide range of activating ion concentrations. Throughout the study we used the ethanol concentration $(50 \mathrm{mM})$ reached in blood during moderate-to-heavy intoxication. Data demonstrate that

1) Slo1 channel sensitivity to ethanol channel is dependent on $\mathrm{Ca}^{2+}$ but not in $\mathrm{Mg}^{2+}$ presence, even when the latter is present at levels that do activate slo1channels; 2) activating ion i.e., $\mathrm{Ca}^{2+}$ is required for and modulates ethanol regulation of slo1 channel activity, while being unable to embold slo2 or slo3 channels with ethanol sensitivity and 3) our findings strongly suggest that distinct sensitivity of slo1 channels coupled to channel $\mathrm{Ca}^{2+}$ sensing structures.

The ethanol modulation of BK channel (in our case, cbv1) activity by $50 \mathrm{mM}$ ethanol shown in this study is similar to previously published reports with mslo1 channels expressed in X. laevis oocytes (Liu et al. 2003; Brodie et al. 2007; Liu et al. 2008; Bukiya et al. 2009); with hslo1 channels cloned from human brain and expressed in HEK cells (Feinberg-Zadek et al. 2008) or slo1 channels reconstituted into bilayers of one or two phospholipid species (Crowley et al. 2003; Crowley et al. 2005; Yuan et al. 2011). Collectively, it should be underscored that slo1 channel-forming subunit surrounded by a bare phospholipid bilayer is sufficient to render the BK channels sensitive to ethanol (Crowley et al. 2003; Crowley et al. 2005; Yuan et al. 2011).

From a structural point of view, the differential ethanol response of slo1 vs. slo2.1/slo2.2 and slo3 channels is quite remarkable. Similar to all other members of the superfamily of voltage-gated, outward rectifying $\mathrm{K}^{+}$channels, slo1 and slo2 proteins contain a transmembrane core of TM6. Additionally, each slo1 or slo2 channel-forming protein contains an ion-sensing CTD with its two RCKs (Jiang et al. 2002; Yusifov et al. 2008; Lee and Cui 2010; Yuan et al. 2011). In addition, RCK2 domain of rslo2.2 channel contains identification of a $\mathrm{Na}^{+}$-coordination site ("sensor") (Zhang et al. 2010) and RCK domains in Slo1 CTD contains several $\mathrm{Ca}^{2+}$-coordination sites ("sensors"). Therefore, ethanol refractoriness of slo2.1/slo2.2 channels indicates that a CTDcontaining RCK domains that empowers the TM6 core of Kv structure with ion-sensing properties does not necessarily embold the resulting channel with sensitivity to intoxicating levels of ethanol (Table 4-1).

Like their slo1 counterparts, slo3 channels contain an N-terminus to $\mathrm{S} 0-\mathrm{S} 1$ cytosolic loop, the TM6 core and the RCK domains. In addition, RCK domains of slo3 channels contain a $\mathrm{OH}^{-}$-coordination site ("sensor") in their CTD (Xia et al. 2004). Thus, the refractoriness of slo3 channels to ethanol indicates that the $\mathrm{N}$ terminus to the $\mathrm{S} 0-\mathrm{S} 1$ cytosolic loop with an ion sensing portion of RCK domains of CTD that senses the $\mathrm{OH}^{-}$ are not sufficient to empower the TM6 KV core with sensitivity to intoxicating ethanol concentrations. 
Table 4-1. Summary of key structural features of different slo channel family members, their activating ligands and sensitivity to $50 \mathrm{mM}$ ethanol

\begin{tabular}{ccccc}
\hline $\begin{array}{c}\text { Construct/ } \\
\text { Channel }\end{array}$ & So Domain & RCKs & Activating Ion & $\begin{array}{c}\text { Sensitivity to } \\
\mathbf{5 0 ~} \mathbf{~ m M} \\
\text { Ethanol }\end{array}$ \\
\hline Cbv1 & Present & Present & $\mathrm{Ca}^{2+}{ }_{i}$ & Yes \\
Cbv1 & Present & Present & $\mathrm{Mg}^{2+}{ }_{i}$ & No \\
Slo2 & Absent & Present & $\mathrm{Na}^{2+}$ & No \\
Slo3 & Present & Present & $\mathrm{OH}^{-}$ & No \\
\hline
\end{tabular}

Source: Modified with permission from Liu, J., Bukiya, A.N., Kuntamallappanavar, G., Singh, A.K. and Dopico, A.M. (2013). Disntict sensitivity of Slo1 channel proteins to ethanol. Mol. Pharmacol 83(1): 235-244. 
Collectively, our results from mslo1, rslo2.1/2.2 and mslo3 channels led us to conclude that the slo family canonical design, which includes a TM6 voltage-dependent $\mathrm{K}^{+}\left(\mathrm{K}_{\mathrm{V})}\right.$ core and CTDs that empower the channel with ion-sensing, does not necessarily render the channel protein sensitive to intoxicating concentrations of ethanol. Rather, we speculated that the presence of a $\mathrm{Ca}^{2+}$ - sensing machinery and thus $\mathrm{Ca}^{2+}$-driven gating seem to be essential for ethanol modulation of slo1 channel activity (Liu et al., 2008). Thus, $50 \mathrm{mM}$ ethanol seems to differentially sense the coupling between different ions $\left(\mathrm{Ca}^{2+}\right.$ versus $\left.\mathrm{Na}^{+}, \mathrm{OH}^{-}\right)$and closed to open transition of conserved $\mathrm{K}^{+}$pore domain.

Previous studies demonstrated that slo1 channels are activated by two fairly independent gating "pathways": $\mathrm{Ca}^{2+}$ and $\mathrm{Mg}^{2+} / \mathrm{V}$-dependent pathways (Shi et al. 2002), which have been linked to the involvement of at least two different structural domains in determining channel activity. A low-affinity divalent cation-binding site is responsible for $\mathrm{Mg}^{2+}$-dependent pathway, located near amino terminus of RCK1 domains. Whereas, high-affinity $\mathrm{Ca}^{2+}$ binding site and $\mathrm{Ca}^{2+}{ }_{i}$ bowl regions are necessary for $\mathrm{Ca}^{2+}$-dependent pathway activation, which is located at carboxy terminus of RCKs. It is thought that S6RCK "linker" that connects S6 to RCKs serves as a passive spring during $\mathrm{Ca}^{2+}{ }_{\mathrm{i}}$-or $\mathrm{Mg}^{2+}{ }_{\mathrm{i}}$ dependent activation (Niu et al. 2004). It is evident from previous and current studies that ethanol fails to alter: 1) voltage-driven (Dopico et al. 1998; Dopico et al. 1999; Liu et al. 2008). 2) $\mathrm{Mg}^{2+}$-driven gating (present study) and 3) cannot modify the slo1 channel activity in the absence of $\mathrm{Ca}^{2+}$ (present study and Liu et al., 2013). From structural point of view, these results suggest that ethanol 1) does not alter major aspects of gating involving voltage sensors 2) does not affect the gating involving low-affinity divalent cation-binding site 3) these data seem to suggest that ethanol does not modify the behavior of peptidic "springs" that are connecting RCK domains to S6 gate as previously claimed (Niu et al. 2004; Treistman and Martin 2009). Rather, ethanol 4) modulates gating involving either the calcium bowl or RCK1 high-affinity site to modulate channel activity and thus current. Further, previous studies demonstrated that mutations of residues (Glu912 and Asp923) in $\mathrm{Ca}^{2+}$ bowl did not modulate the ethanol-induced activation or inhibition of slo1 channel (Liu et al. 2008). However, mutations (Asp362 and Asp367) in high affinity RCK1 site (Xia et al. 2002), abolished ethanol-mediated inhibition of the slo1 channel yet did not alter drug-induced channel potentiation. Interestingly, combining both the mutations 5D5N and D362A/D367A not only abolished the ethanol-induced inhibition but also abolished activation due to ethanol (Liu et al. 2008). Collectively, these results indicate that as far as $\mathrm{Ca}^{2+}{ }_{\mathrm{i}}$ able to reach one of its two high affinity recognition sites in the slo1 CTD, ethanol is able to modulate slo1 channel activity. 


\section{CHAPTER 5. ETHANOL DISTINCTLY MODULATES BETA1 AND BETA2- CONTAINING BK CHANNELS}

\subsection{Overview}

Voltage- and $\mathrm{Ca}^{2+}{ }^{2}$-gated, large conductance $\mathrm{K}^{+}(\mathrm{BK})$ channels control several physiological processes, participate in the pathophysiology of disease and are considered useful therapeutic targets (Brenner et al. 2000; Jaggar and Nelson 2000; Jaggar et al. 2000; Pluger et al. 2000; Erdos et al. 2002; Wellman et al. 2002; Dopico et al. 2012). Functional BK channels result from tetrameric association of $\alpha$ subunits. The basic BK channel phenotype, voltage-, $\mathrm{Ca}^{2+}$-, and $\mathrm{Mg}^{2+}$-driven gating is replicated when slo1 homotetramers are expressed in a variety of mammalian and non-mammalian systems (Brenner et al. 2000; Cox and Aldrich 2000; Bukiya et al. 2009). However, several key biophysical and pharmacological properties are drastically modulated by the presence of accessory $\beta$ subunits $(\beta 1-\beta 4)$. Most importantly, some $\beta$ subunits are known to modulate the apparent $\mathrm{Ca}^{2+}{ }_{i}$ sensitivity of the channel (Behrens et al. 2000; Brenner et al. 2000; Cox and Aldrich 2000; Bao and Cox 2005; Orio and Latorre 2005; Sun et al. 2013).

Acute exposure to ethanol concentrations obtained in human blood during alcohol intoxication (10-100 mM) modulates BK (slo1) channel steady-state activity $\left(\mathrm{NP}_{\mathrm{o}}\right)$ (Brodie et al. 2007). This ethanol's action on homomeric BK channel is dependent on the presence of the channel's activating ion i.e., $\mathrm{Ca}^{2+}$ i, ethanol final effect on slol channel (potentiation, refractoriness or inhibition) being a function of $\mathrm{Ca}^{2+}{ }_{i}$ concentration (see chapter 4) (Liu et al. 2008; Liu et al. 2013). Several studies reported that $\beta 1$ and $\beta 4$ subunits modulate ethanol action on BK constituted by slo1+beta heteromers (Martin et al. 2004; Liu et al. 2008; Bukiya et al. 2009). Results are conflicting and difficult to interpret because these studies used different slol proteins and experimental conditions, including levels of $\mathrm{Ca}^{2+}$ i. Both slol channels isoforms (Dopico 2003; Liu et al. 2003; Liu et al. 2006) and $\mathrm{Ca}^{2+}{ }_{\mathrm{i}}$ levels (Liu et al. 2008) are known to influence ethanol action on BK channels. To systematically probe the role of different beta subunits in ethanol action on BK channels and understand the underlying common and different gating mechanisms involved targeted by the drug, we expressed cbv1 (slo1) alone or with $\beta 1, \beta 2, \beta 2$-IR, $\beta 3$ or $\beta 4$, and conducted $\mathrm{G} / \mathrm{G}_{\max }-\mathrm{V}$ plots in absence and presence of $50 \mathrm{mM}$ ethanol across a wide range of $\mathrm{Ca}^{2+}{ }_{\mathrm{i}}(0-100 \mu \mathrm{M})$. Further, the resulting plots were fitted with 70 -state HA model. My study tested the following hypotheses.

- Ethanol differentially modulates BK current from channel complexes containing different beta subunits.

- Ethanol action on different $\beta$-containing BK channels depends on extent to which these accessory subunits modulate apparent change in $\mathrm{Ca}^{2+}{ }_{i}$-sensitivity.

- Ethanol modulates specific aspects of channel gating, which facilitates inhibition of current from $\beta 1$-containing BK channel complexes 


\subsection{Results}

\subsubsection{Auxillary Beta Subunits Functionally Co-Express with Channel Forming Cbv1 Subunits}

Before probing ethanol on BK channels of different subunit composition, we determined the functional co-expression of the different beta subunits $(\beta 1, w t \beta 2, \beta 2$-IR, $\beta 3$ and $\beta 4$ ) with the channel-forming cbv1 subunit. Thus, using the oocyte expression system, macroscopic currents were evoked from a holding potential of $-80 \mathrm{mV}$ by 100 $\mathrm{ms}-$ long, $10 \mathrm{mV}$ depolarizing steps from $-150 \mathrm{mV}$ to $+150-200 \mathrm{mV}$. Macroscopic recordings were obtained from inside-out patches over a wide range of $\mathrm{Ca}^{2+}{ }_{\mathrm{i}}$ concentrations (nominal zero-100 $\mu \mathrm{M}$ ) (Figure 5-1A). To identify the ion current phenotype from the different BK channel complexes, we obtained $\mathrm{G} / \mathrm{G}_{\max }-\mathrm{V}$ plots, $\mathrm{V}_{0.5^{-}}$ $\left[\mathrm{Ca}^{2+}\right]$ plots, time constant for ion current activation $\left(\tau_{\mathrm{act}}\right)$ and time constant for ion current deactivation $\left(\tau_{\text {deact }}\right)$ from each macroscopic current recording. $\tau_{\text {act }}$ and $\tau_{\text {deact }}$ were measured at the voltage at which the channel reached maximal conductance $\left(\mathrm{V}_{\max }\right)$.

As previously reported for other slo1 channels (Brenner et al. 2000; Cox and Aldrich 2000; Bao and Cox 2005; Orio and Latorre 2005), the presence of $\beta 1$ subunits increased cbv1 channel activity as $\mathrm{Ca}^{2+}{ }_{i}$ was raised above micro molar levels, which is evident by increased shift in $\mathrm{V}_{0.5}$ values in $\mathrm{V}_{0.5}-\mathrm{Ca}^{2+}$ plots (Figure 5-1B). These results are in agreement with previously published reports (Brenner et al. 2000; Cox and Aldrich 2000; Bao and Cox 2005; Orio and Latorre 2005; Contreras et al. 2012). It should be underscored that $\mu \mathrm{M} \mathrm{Ca}^{2+}{ }_{i}$ levels, while not only necessary, are optimal for $\beta 1$ subunit to modulate slo1 channels so such modulation translates into observable increase in $\mathrm{NP}_{\mathrm{o}}$ and/or macroscopic conductance. More specifically, $\beta 1$ subunit-induced channel modulation results in an increased apparent $\mathrm{Ca}^{2+}$ sensitivity of the channel and thus enhanced steady-state activity/current (Brenner et al. 2000; Cox and Aldrich 2000; Nimigean and Magleby 2000; Bao and Cox 2005; Orio and Latorre 2005; Contreras et al. 2012). In addition, $\beta 1$ subunit increased $\tau_{\text {act }}$ and $\tau_{\text {deact }}$ from their cbv1 values: at $10 \mu \mathrm{M}$ $\mathrm{Ca}^{2+}{ }_{\mathrm{i}} . \tau_{\text {act }}$ and $\tau_{\text {deact }}$ changed from $1.40 \pm 0.10$ and $1.13 \pm 0.13 \mathrm{~ms}$ to $5.16 \pm 0.93$ and $6.76 \pm 0.67 \mathrm{~ms}$, for cbv1 and cbv1 $+\beta 1$, respectively $(\mathrm{p}<0.05$ for both constants $)$ (Figure 5-1C). This slowing down of macroscopic current activation and deactivation kinetics by BK $\beta 1$ subunits is also in accordance with data from slo1 other than cbv1 (Brenner et al. 2000; Cox and Aldrich 2000; Bao and Cox 2005; Contreras et al. 2012).

From a structural point of view, wt $\beta 2$ subunits possess an additional inactivation bowl/peptide in their N-terminus when compared to $\beta 1$ subunits (Wallner et al. 1995; Wallner et al. 1999; Xia et al. 1999; Uebele et al. 2000; Xia et al. 2003). Indeed, coexpression of wt $\beta 2$ with cbv1 channels consistently resulted in remarkably rapid inactivation of ionic current (Figure 5-1A, bottom left panel). This finding is consistent with findings from other laboratories that co-expressed wt $\beta 2$ with slo1 other than cbv1 (Wallner et al. 1999; Xia et al. 1999; Uebele et al. 2000; Xia et al. 2003), and confirms previous data from our laboratory obtained with cbv1+wt $\beta 2$ channel expressed in $X$. laevis oocytes (Bukiya et al. 2009). 

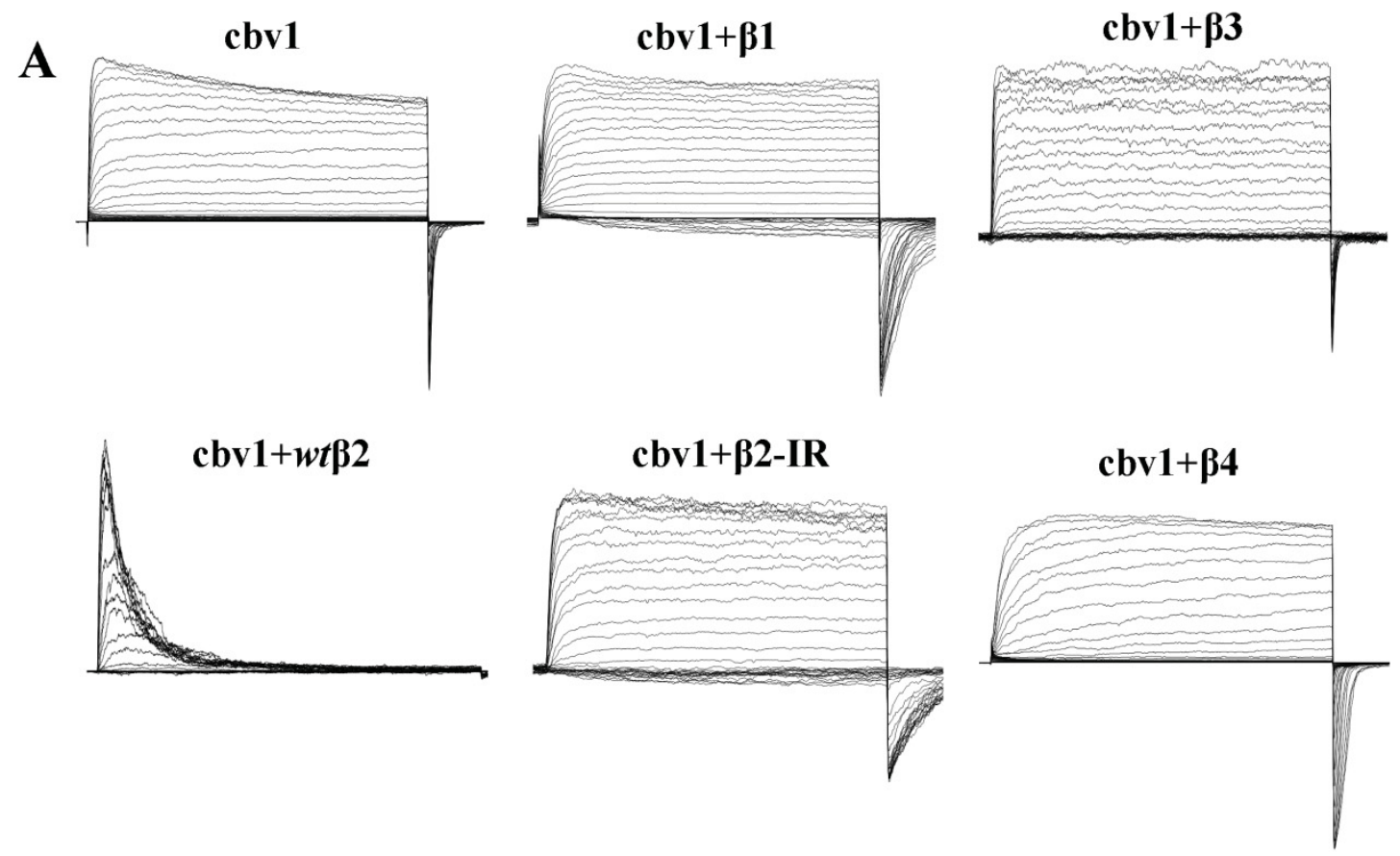

B

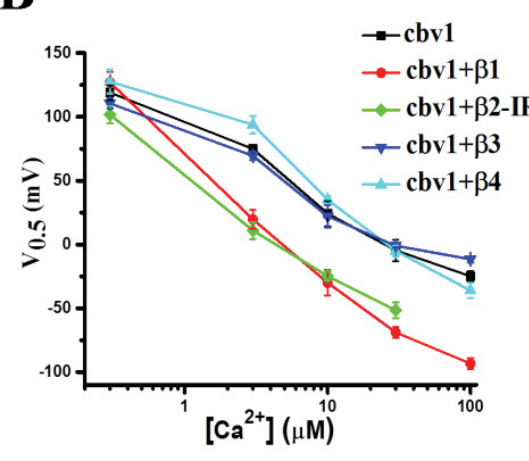

C

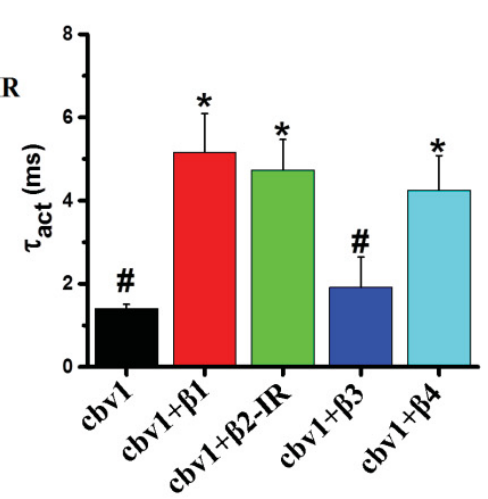

D

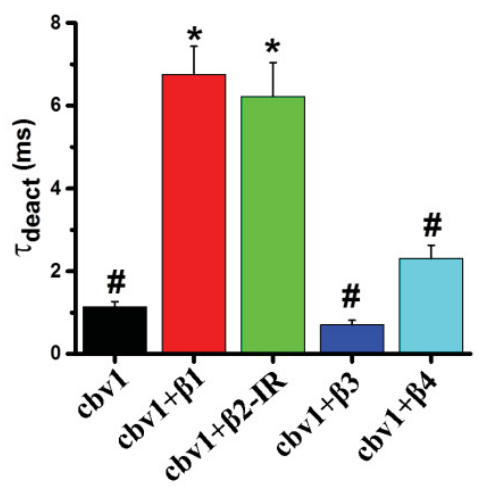

Figure 5-1. Macroscopic currents after cbv1+ $\beta 1-4$ subunit expression in Xenopus laevis oocytes show characteristic features of BK currents

Notes: A) Representative current records from $\mathrm{I} / \mathrm{O}$ macropatches expressing different $\mathrm{BK}$ channel subunit combinations (cbv1 $1 \beta 1$, wt $\beta 2, \beta 2$-IR, $\beta 3$, or $\beta 4)$. Currents were evoked by $100 \mathrm{~ms}-$ long (except for cbv1+wt $\beta 2 ; 600 \mathrm{~ms}-\mathrm{long}$ ), $10 \mathrm{mV}$ depolarizing steps from -150 to $+150 \mathrm{mV}$, with a holding potential set to $-80 \mathrm{mV}$. cbv1+wt $\beta 2$ channels are characterized by fast inactivation. B) $\mathrm{V}_{0.5} v s$. $\left[\mathrm{Ca}^{2+}{ }_{i}\right]$ plot underscoring that the presence of different $\beta$ subunits differentially modulate the apparent $\mathrm{Ca}^{2+}{ }_{i}$ sensitivity of the channel complex. C) and D) Bar graphs show averaged activation (C) and deactivation (D) time constants $\left(\tau_{\text {act }}, \tau_{\text {deact }}\right.$, respectively) obtained at $\mathrm{V}_{\max }$ at $\mathrm{Ca}^{2+}{ }_{\mathrm{i}}=10 \mu \mathrm{M}$. Each data point represents the average of no less than 3 patches, each excised from a different cell. *Different from cbv1 $(\mathrm{p}<0.05)$; \#Different from cbv1+ $\beta 1$. 
As found with $\beta 1$ subunit, co-expression of $\beta 2$-IR subunit with cbv1 produced an increase in the channel's apparent $\mathrm{Ca}^{2+}{ }_{i}$ sensitivity (Figure 5-1B). Further, when compared to homomeric cbv1 channels, the presence of $\beta 2$-IR subunit induced remarkable slowdown of activation and deactivation kinetics, as previously shown by other researchers with slo1 channels other than cbv1 (Brenner et al. 2000; Orio and Latorre 2005; Sun et al. 2013). Indeed, at $10 \mu \mathrm{M} \mathrm{Ca}^{2+}{ }_{i}, \tau_{\text {act }}$ and $\tau_{\text {deact }}$ changed from $1.40 \pm 0.10$ and $1.13 \pm 0.13 \mathrm{~ms}$ to $4.73 \pm 0.74$ and $6.22 \pm 0.82 \mathrm{~ms}$, for cbv1 and cbv1 $+\beta 2-\mathrm{IR}$, respectively $(\mathrm{P}<0.05$ for both constants) (Figure 5-1C and $\mathbf{D})$.

In contrast to all other $\beta$ subunits, functional co-expression of $\beta 3$ subunits are characterized by their lack of modulation of apparent change in $\mathrm{Ca}^{2+}{ }_{i}$ sensitivity, activation and deactivation kinetics. At $10 \mu \mathrm{M}, \tau_{\text {act }}$ and $\tau_{\text {deact }}$ values changed from $1.40 \pm 0.10$ and $1.13 \pm 0.13 \mathrm{~ms}$ to $1.91 \pm 0.73$ and $0.76 \pm 0.11 \mathrm{~ms}$, for cbv1 and cbv $1+\beta 3$, respectively (Figure 5-1C and D). These data are in agreement with previously published reports from other laboratories (Behrens et al. 2000; Brenner et al. 2000; Uebele et al. 2000).

In contrary to $\beta 1$ and $\beta 2-\mathrm{IR}$, expression of $\beta 4$ increased cbv1 $\mathrm{V}_{0.5}$ at $0.3-10 \mu \mathrm{M}$ $\mathrm{Ca}^{2+}{ }_{\mathrm{i}}$ while mildly decreasing $\mathrm{V}_{0.5}$ at $30-100 \mu \mathrm{M} \mathrm{Ca}^{2+}{ }_{\mathrm{i}}$ (Figure 5-1B). These results indicate modulation of $\mathrm{Ca}^{2+}{ }_{i}$ sensitivity by $\beta 4$ subunit requires more than $20 \mu \mathrm{M} \mathrm{Ca}^{2+}{ }_{\mathrm{i}}$. In addition, at $10 \mu \mathrm{M} \mathrm{Ca}^{2+}{ }_{i}, \beta 4$ remarkably increased $\tau_{\text {act }}: 1.40+0.1 \mathrm{~ms}$ in cbv1 vs. $4.25 \pm 0.83$ $\mathrm{ms}$ in cbv $1+\mathrm{h} \beta 4(\mathrm{P}<0.05)$ while mildly increasing $\tau_{\text {deact }}$ when compared to cbv1 channels (Figure 5-1C and D). Collectively, the $\beta 4$-introduced changes in $\mathrm{V}_{0.5}, \tau_{\text {act }}$ and $\tau_{\text {deact }}$ over cbv1 values are consistent with those previously reported with $\beta 4$ and slo1 channels other than cbv1 (Behrens et al. 2000; Brenner et al. 2000; Orio et al. 2002).

\subsection{2. $\beta 1$ Subunit Facilitates Ethanol-Induced Inhibition of $\beta 1-C o n t a i n i n g ~ B K$ Channels}

Ethanol action on slo1/cbv1 channels is a function of $\mathrm{Ca}^{2+}{ }_{\mathrm{i}}$ (Liu et al. 2008; Bukiya et al. 2009) (see chapter 4). Several laboratories, including ours, demonstrated that $\beta 1$ subunit increases apparent $\mathrm{Ca}^{2+}{ }_{\text {i }}$ sensitivity of the BK channel (Brenner et al. 2000; Cox and Aldrich 2000; Bao and Cox 2005; Orio and Latorre 2005; Bukiya et al. 2009; Kuntamallappanavar et al. 2014). Thus, we set to address the role of $\beta 1$ subunit on ethanol modulation of BK channel activity, with a focus on $\mathrm{Ca}^{2+}$-driven gating processes.

After characterizing functional expression of cbv1+ $\beta 1$ channels, $50 \mathrm{mM}$ ethanol was applied onto the cytosolic side of these channels. All experimental conditions, data acquisition and analysis were similar to those used in our studies with cbv1 channels. Results showed that ethanol activated cbv1 $+\beta 1$ channels at low $\left[\mathrm{Ca}^{2+}{ }_{i}\right]$ i.e., $0.3 \mu \mathrm{M}$, which is evident as is a shift to the left in the $\mathrm{G} / \mathrm{G}_{\max }-\mathrm{V}$ plots and thus significantly decreased $\mathrm{V}_{0.5}$ values in the $\mathrm{V}_{0.5}-\left[\mathrm{Ca}^{2+}\right]$ plot (Figure 5-2A and $\mathbf{B}$ ). As $\left[\mathrm{Ca}^{2+}{ }_{i}\right]$ increased, this activation turned to significant inhibition, which is evident as a significant right-shift in the $\mathrm{G} / \mathrm{G}_{\max }-\mathrm{V}$ plot (Figure 5-2C) and thus increased $\mathrm{V}_{0.5}$ values in the $\mathrm{V}_{0.5}-\left[\mathrm{Ca}^{2+}{ }_{i}\right]$ plot (Figure 5-3A and B). 

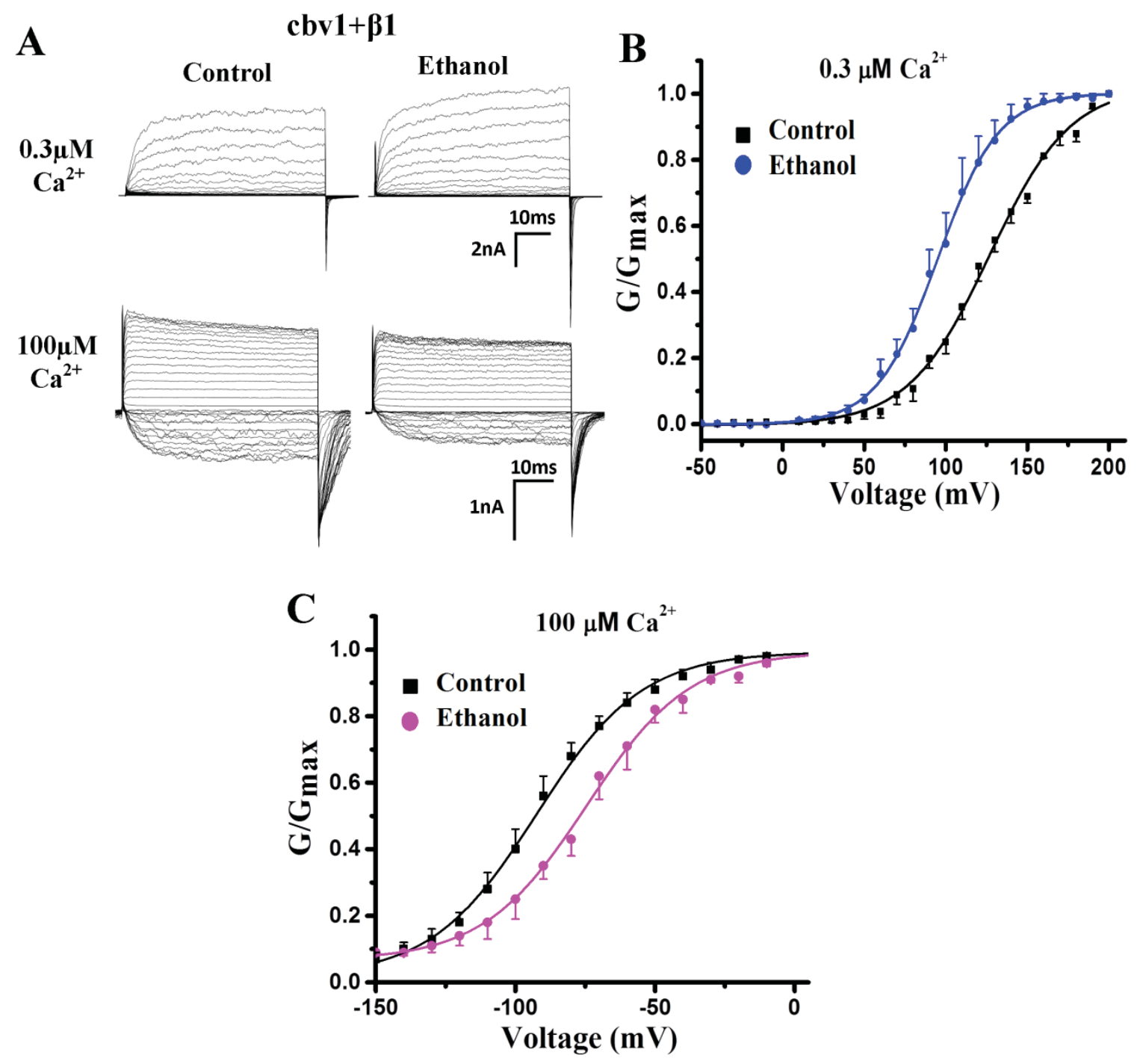

Figure 5-2. Ethanol activates $\beta 1$-containing BK channels at submicromolar (0.3 $\mu \mathrm{M}) \mathrm{Ca}^{2+}$, while causing strong inhibition at higher $(100 \mu \mathrm{M}) \mathrm{Ca}^{2+}{ }_{\mathrm{i}}$

Notes: A) Macroscopic current recordings from I/O patches obtained at 0.3 and $100 \mu \mathrm{M}$ $\mathrm{Ca}^{2+}{ }_{\mathrm{i}}$ in the absence or presence of $50 \mathrm{mM}$ ethanol after cbv $1+\beta 1$ expression in Xenopus oocytes. Ethanol shifts $\mathrm{G} / \mathrm{G}_{\max }-\mathrm{V}$ plot to the left at $0.3 \mu \mathrm{M} \mathrm{Ca}^{2+}{ }_{\mathrm{i}}$ indicating BK current potentiation (B) while shifting the plot to the right at $100 \mu \mathrm{M} \mathrm{Ca}^{2+}{ }_{\mathrm{i}}$ indicating inhibition (C). Each data point represents the average of no less than 3 patches, each excised from a different cell. 


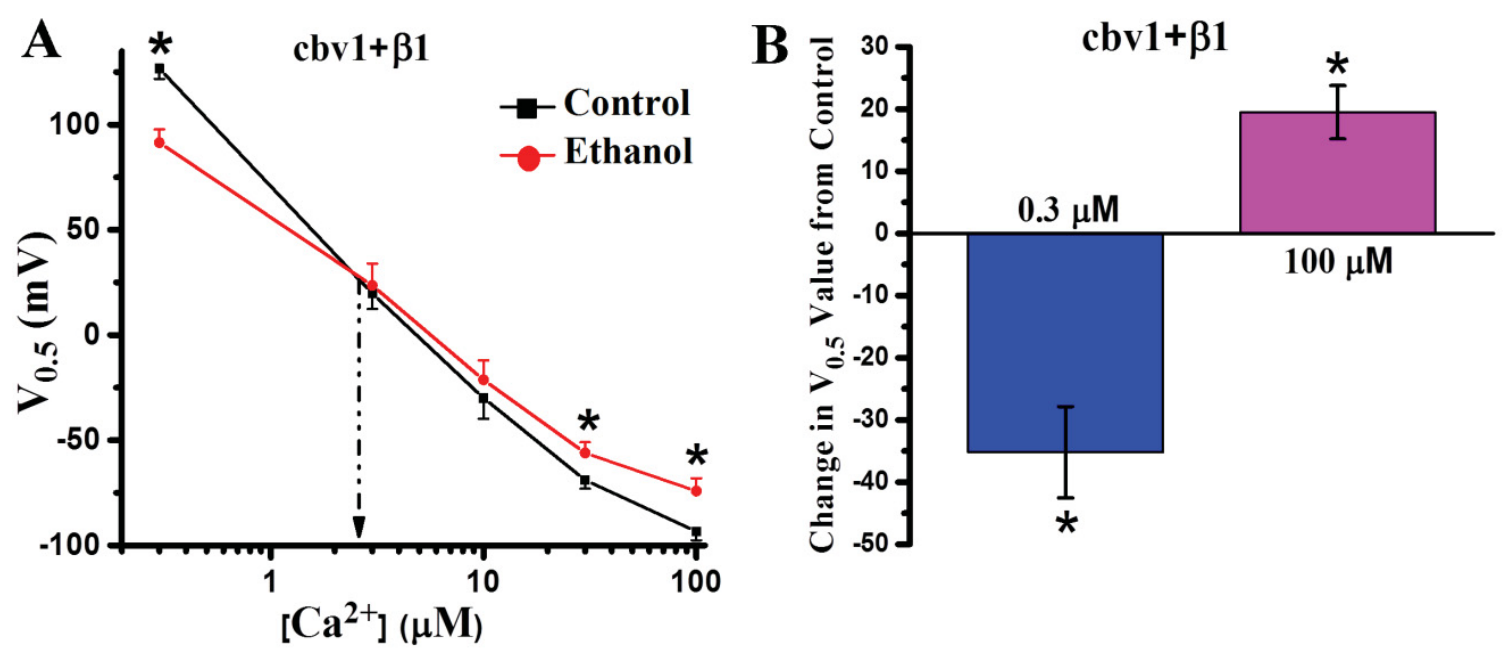

Figure 5-3. BK $\beta 1$ subunits determine ethanol-induced inhibition of BK channels at physiological $\mathrm{Ca}^{2+}{ }_{\mathrm{i}}(3-45 \mu \mathrm{M})$ and voltages $(-60$ to $-20 \mathrm{mV})$

Notes: A) $\beta 1$ subunits set the activation-to-inhibition crossover of ethanol responses at $\approx 3$ $\mu \mathrm{M} \mathrm{Ca}^{2+}{ }_{\mathrm{i}}$. B) Bar graph representing ethanol-induced change in $\mathrm{V}_{0.5}$ values from preethanol application obtained at $0.3 \mu \mathrm{M}$ and $100 \mu \mathrm{M} \mathrm{Ca}^{2+}{ }_{i}$. Each data point represents the average of no less than 3 patches, each excised from a different cell. *Different from control $(\mathrm{p}<0.05)$. 
Further, ethanol-induced activation-to-inhibition crossover shifted to $\sim 3 \mu \mathrm{M}$ (Figure 5-3B) when compared to that of cbv1 channels. It should be underscored that ethanol-mediated inhibition of $\beta 1$-containnig BK channels occurs at physiological $\mathrm{Ca}^{2+}{ }_{i}$ and voltage found near cerebral artery myocyte (Perez et al. 2001).

\subsubsection{Ethanol Modulation of $w t \beta 2$ - and $\beta 2$-IR-Containing BK Channel Activity Is Similar to the Alcohol Response of $\beta 1$ Subunit-Containing BK Channels}

Our next aim was to determine the role of $w t \beta 2$ and $\beta 2$-IR subunits in ethanol modulation of BK channel activity.

First we co-expressed wt $\beta 2$ subunits with cbv1 channels. In these experiments, we obtained ionic currents at single channel resolution from inside-out patches in gapfree recording at $\mathrm{V}_{\mathrm{m}}=-20$ to $-40 \mathrm{mV}$ for 30 seconds under each experimental condition (control vs. $50 \mathrm{mM}$ ethanol). Similar to previous studies, $50 \mathrm{mM}$ ethanol was probed over a wide $\mathrm{Ca}^{2+}{ }_{i}$ concentration range (nominal zero-100 $\mu \mathrm{M}$ ). Results were analyzed using a built-in function of Clampfit 9.2. $\mathrm{NP}_{\mathrm{o}}$, an index of channel steady-state activity, represents product of number of channels in the membrane patch $(\mathrm{N})$ and individual channel open probability (Po). Results demonstrated that ethanol activated cbv1+wt $\beta 2$ channel at low $\left[\mathrm{Ca}^{2+}\right]_{\text {i }}$, i.e., $0.3 \mu \mathrm{M}$, which was evident as a significant increase in $\mathrm{NP}_{\mathrm{o}}$ (Figure 5-4A and C). As $\left[\mathrm{Ca}^{2+}\right]$ increased, this activation turned into significant inhibition, which was evident as a significant reduction in $\mathrm{NP}_{\mathrm{o}}$ (Figure 5-4B and $\mathbf{C}$ ). Furthermore, as found with cbv1+ $\beta 1$ channels (Figure 5-3A), ethanol-induced activationto-inhibition cross-over occurred at $\sim 3 \mu \mathrm{M}$ (Figure 5-4C).

Since $w t \beta 2$ subunits contain an inactivation domain, we asked whether ethanol modulation of BK channels by $w t \beta 2$ subunits was due to the presence of the inactivation peptide. Thus, we constructed a $\beta 2$ subunit with its inactivation domain removed (" $\beta 2$ IR). It should be underscored that the $\beta 2$-IR-induced changes in the biophysical properties of BK channels, such as apparent increase in $\mathrm{Ca}^{2+}{ }_{i}$ sensitivity, are very similar to those induced by $\beta 1$ subunits (Behrens et al. 2000; Brenner et al. 2000; Orio et al. 2002). Once the functional expression of $\beta 2$-IR subunit was confirmed, effect of ethanol was explored under experimental conditions similar to those used in cbv $1+\beta 1$ studies. Results demonstrated that ethanol activated cbv1 $+\beta 2$-IR channels at low $\left[\mathrm{Ca}^{2+}\right]_{\text {i }}$, i.e., 0.3 $\mu \mathrm{M}$, which was evident as a marked decrease in $\mathrm{V}_{0.5}$ values from the $\mathrm{V}_{0.5^{-}}\left[\mathrm{Ca}^{2+}\right]$ plot (Figure 5-5A and B). As $\left[\mathrm{Ca}^{2+}\right]_{\mathrm{i}}$ increased, this activation turned to significant inhibition, which coud be noticed from a significant increase in $\mathrm{V}_{0.5}$ values in the $\mathrm{V}_{0.5^{-}}$ $\left[\mathrm{Ca}^{2+}\right]_{\text {i }}$ plot (Figure 5-5A and B). Furthermore, ethanol-induced activation-to-inhibition cross-over shifted to $\sim 3 \mu \mathrm{M}$ (Figure 5-5A), a value similar to that found in cbv1+ $\beta 1$ channels exposed to ethanol (Figure 5-3A). These results seem to indicate that the $\beta 2$ inactivation domain does not play a significant role in ethanol action on cbv1+wt $\beta 2$ channels. 

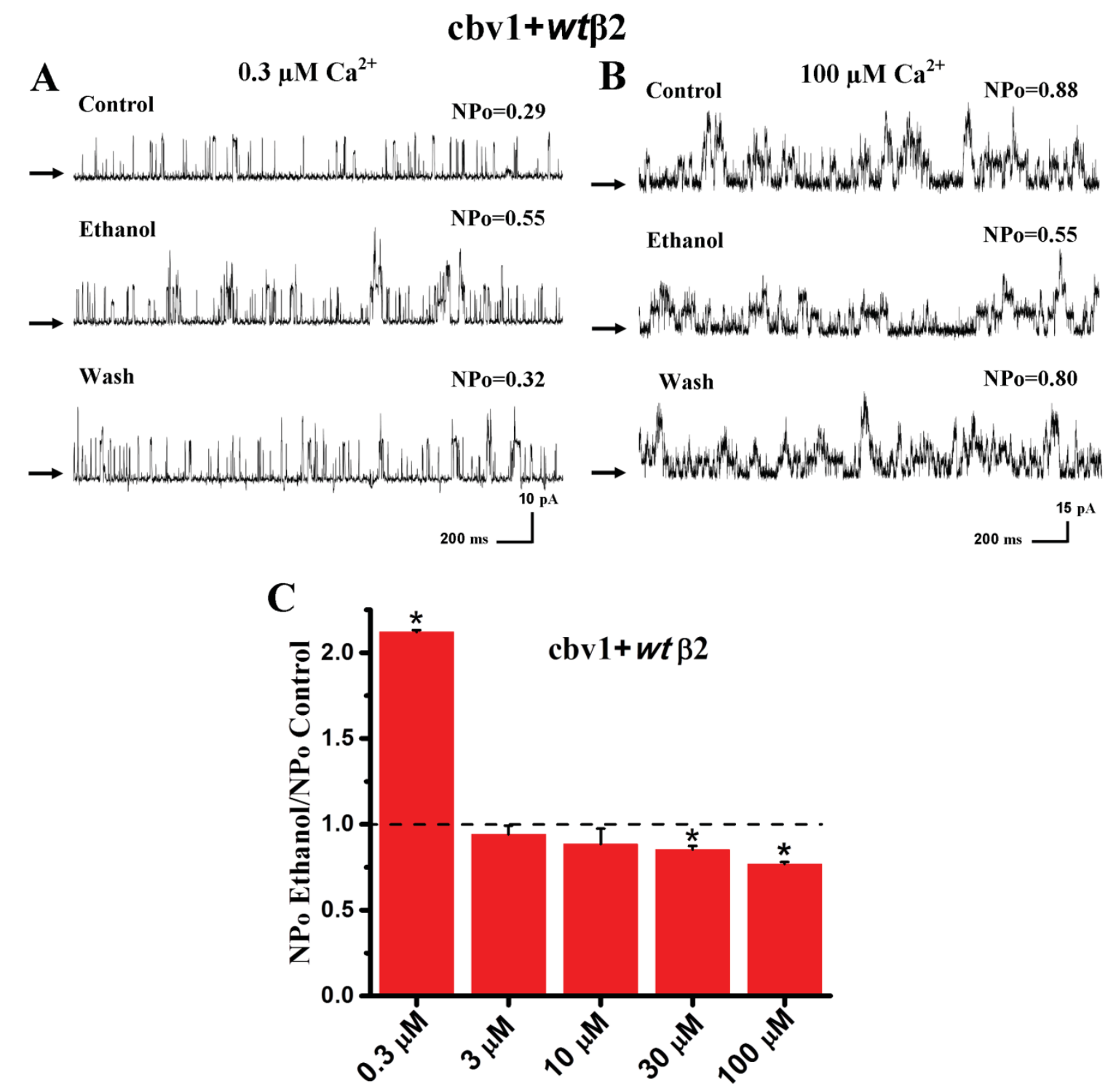

Figure 5-4. Ethanol-mediated inhibition of wt $\beta 2$-containing BK channels is similar to the ethanol response of cbv1+ $\beta 1$ channels

Notes: A) Single-channel recordings of cbv1+wt $\beta 2$ channels from $\mathrm{I} / \mathrm{O}$ patches at 0.3 (left panel) and $100 \mu \mathrm{M} \mathrm{Ca}^{2+}$ (right panel); $\mathrm{V}_{\mathrm{m}}=-40 \mathrm{mV}$. Records were obtained before (top trace on each panel), during (middle trace) and immediately after (bottom trace) patch exposure to $50 \mathrm{mM}$ ethanol. Arrows indicate the baseline (all channels closed). B) Averaged $\mathrm{NP}_{\mathrm{o}}$ ratios in presence and absence of ethanol from cbv1+wt $\beta 2$ at $0.3,3,10$, 30 , and $100 \mu \mathrm{M} \mathrm{Ca}^{2+}{ }_{\mathrm{i}}(\mathrm{n}=5)$. Data demonstrate that the activation-to-inhibition crossover for ethanol effect on cbv1 $+w t \beta 2$ channels occurs at $\approx 3 \mu \mathrm{M} \mathrm{Ca}^{2+}{ }_{i}$. Each data point represents the average of no less than 3 patches, each excised from a different cell. $*$ Different from control $(\mathrm{p}<0.05)$. 


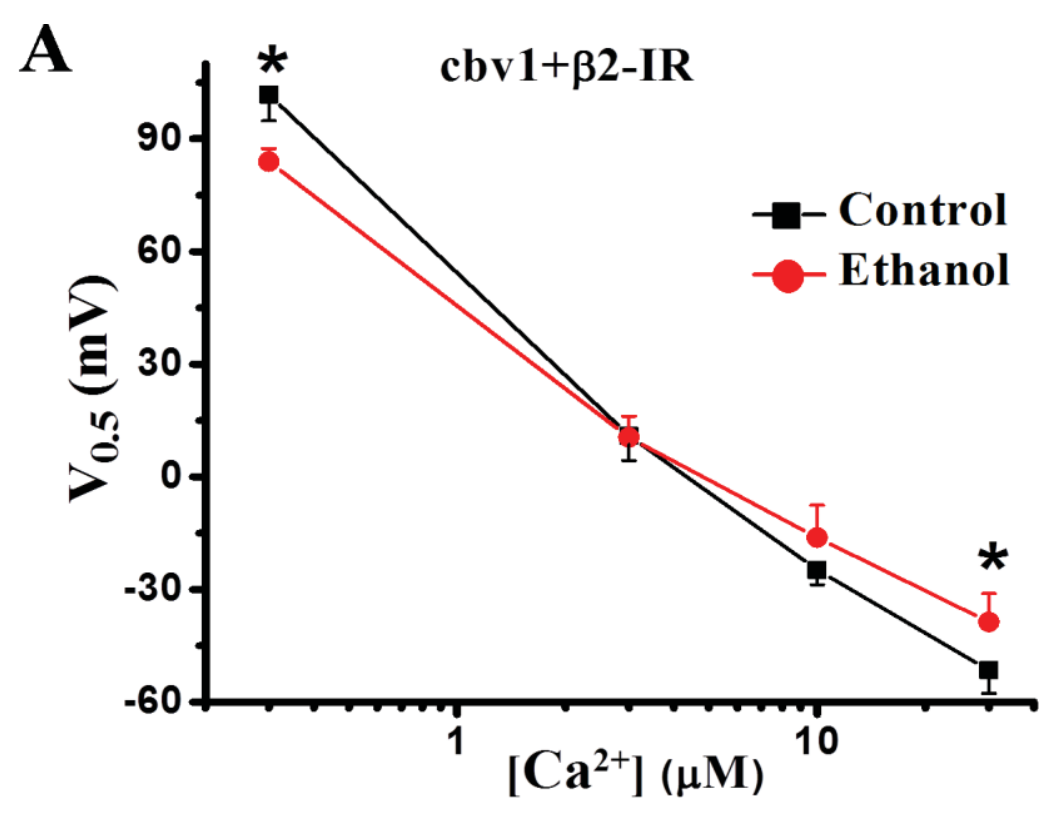

B

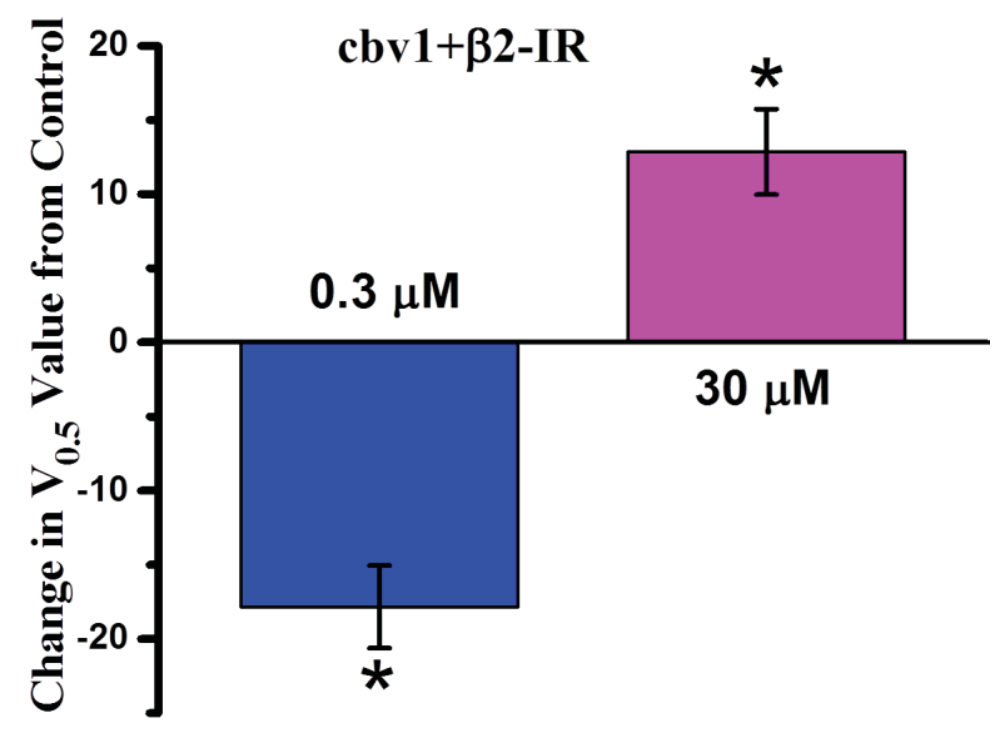

Figure 5-5. Ethanol-mediated inhibition of $\beta 2$-IR-containing BK channels is similar to the ethanol response of cbv1+ $\beta 1$ channels

Notes: A) $\mathrm{V}_{0.5} v s .\left[\mathrm{Ca}^{2+}{ }_{i}\right]$ plot showing that the activation-to-inhibition crossover for ethanol effect on cbv1+ $\beta 2$-IR channels occurs at $\approx 2 \mu \mathrm{M} \mathrm{Ca}^{2+}{ }_{\mathrm{i}}$., as found with cbv1+/beta1 (Figure 5-3A) B) Bar graph showing ethanol-induced change in $\mathrm{V}_{0.5}$ values from control obtained at $0.3 \mu \mathrm{M}$ and $100 \mu \mathrm{M} \mathrm{Ca}^{2+}{ }_{\mathrm{i}}$. Each data point represents the average of no less than 3 patches, each excised from a different cell. *Different from control $(\mathrm{p}<0.05)$. 


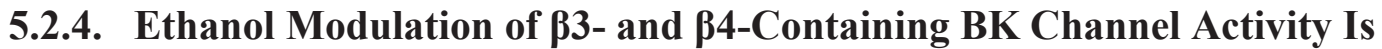 Similar to the Alcohol Response of Homomeric Cbv1 Channels}

Several groups, including our laboratory, documented that unlike $\beta 1$ and $\beta 2$ subunits, $\beta 3$ and $\beta 4$ subunits have a minor, if any, effect on the apparent $\mathrm{Ca}^{2+}{ }_{\text {i }}$ sensitivity of slo1 channels (Behrens et al. 2000; Brenner et al. 2000; Bao and Cox 2005; Orio and Latorre 2005; Bukiya et al. 2009; Sun et al. 2013; Kuntamallappanavar et al. 2014) (Figure 5-1B). If $\beta 1$ - and $\beta 2$-induced facilitation of ethanol inhibition of BK currents is somewhat linked to the channel apparent $\mathrm{Ca}^{2+}{ }_{i}$-sensitiivty, we expected that ethanol action on $\beta 3$ - or $\beta 4$-containing BK channels should not differ from ethanol action on homomeric slo1.

Under experimental conditions similar to those of previous studies, cbv $1+\beta 3$ and cbv $1+\beta 4$ were co-expressed and probed with $50 \mathrm{mM}$ ethanol. Results demonstrated that ethanol activated cbv $1+\beta 3$ and cbv1 $+\beta 4$ channels at low $\left[\mathrm{Ca}^{2+}\right]_{i}$ i.e., $0.3 \mu \mathrm{M}$, which was evident as a marked decrease in $\mathrm{V}_{0.5}$ from the $\mathrm{V}_{0.5}-\left[\mathrm{Ca}^{2+}\right]$ plot (Figure 5-6A, B, C and D). As $\left[\mathrm{Ca}^{2+}\right]$ increased, this activation turned into mild inhibition, which could be noticed by a mild increase in $\mathrm{V}_{0.5}$ from the $\mathrm{V}_{0.5}-\left[\mathrm{Ca}^{2+}\right]$ plot (Figure 5-6A, B, C and D). In both heteromeric complexes, ethanol-induced activation-to-inhibition cross-over occurred at $\sim 20 \mu \mathrm{M}$ (Figure 5-6A and $\mathbf{C}$ ). Thus, $\beta 3$ - and $\beta 4$-containing BK channels response to Ethanol matched that of homomeric cbv1 channels, indicating that $\beta 3$ and $\beta 4$ subunits do not noticeably modulate ethanol action on homomeric BK channels.

\subsubsection{Understanding BK Channel Function Requires a Complex Allosteric Model}

The 70-state Horrigan-Aldrich (HA) model is widely considered the most advanced and complete model to understand BK channel function, in particular its allosteric gating (Figure 5-7). Other allosteric models include 10-state MWC (MonodWyman-Changeux) and the 50-state HA models. According to MWC model (Monod et al. 1965), ion channel exists in two conformations i.e., open and closed. Application of this concept to BK channels requires that the equilibrium between these two conformations is voltage-dependent, with depolarization favoring channel opening. The channel, however, is a homotetramer and contains four $\mathrm{Ca}^{2+}$ binding sites, one site in each subunit. $\mathrm{Ca}^{2+}$ can bind to both conformations of channel. Allosteric theory requires that the probability of $\mathrm{Ca}^{2+}$ binding to the open state is much higher than binding to the closed state, stabilizing the channel in the former. Further, the MWC model assumes binding of $\mathrm{Ca}^{2+}$ to each site is equivalent and independent of binding at other sites. This model is presented in the form of cartoon (Scheme A of Figure 5-7).

Although, the MWC model accounts for many aspects of BK channel gating, there are some aspects the model fails to incorporate: 1. movement of gating charges before the channel opens (Stefani et al. 1997; Horrigan and Aldrich 1999); 2. the complex relation between deactivation time constant and membrane voltage (Cox et al. 1997; Cox et al. 1997; Horrigan and Aldrich 1999). 


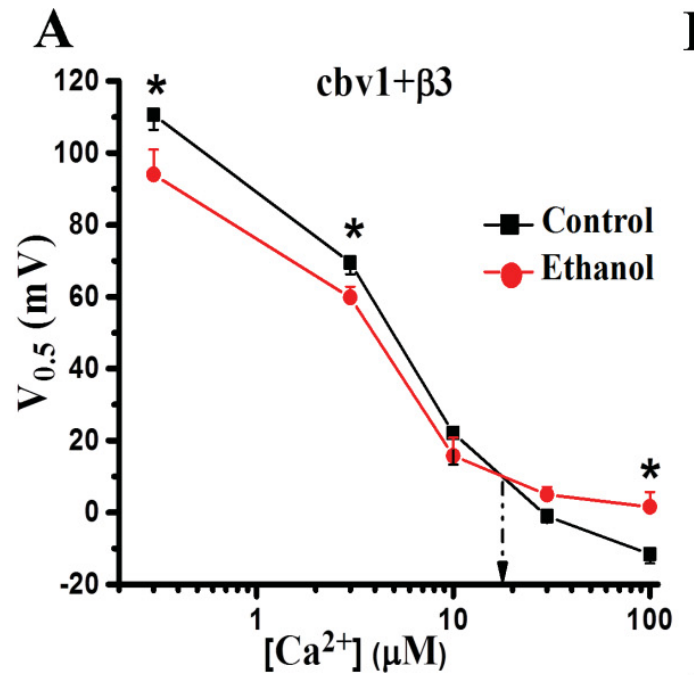

B
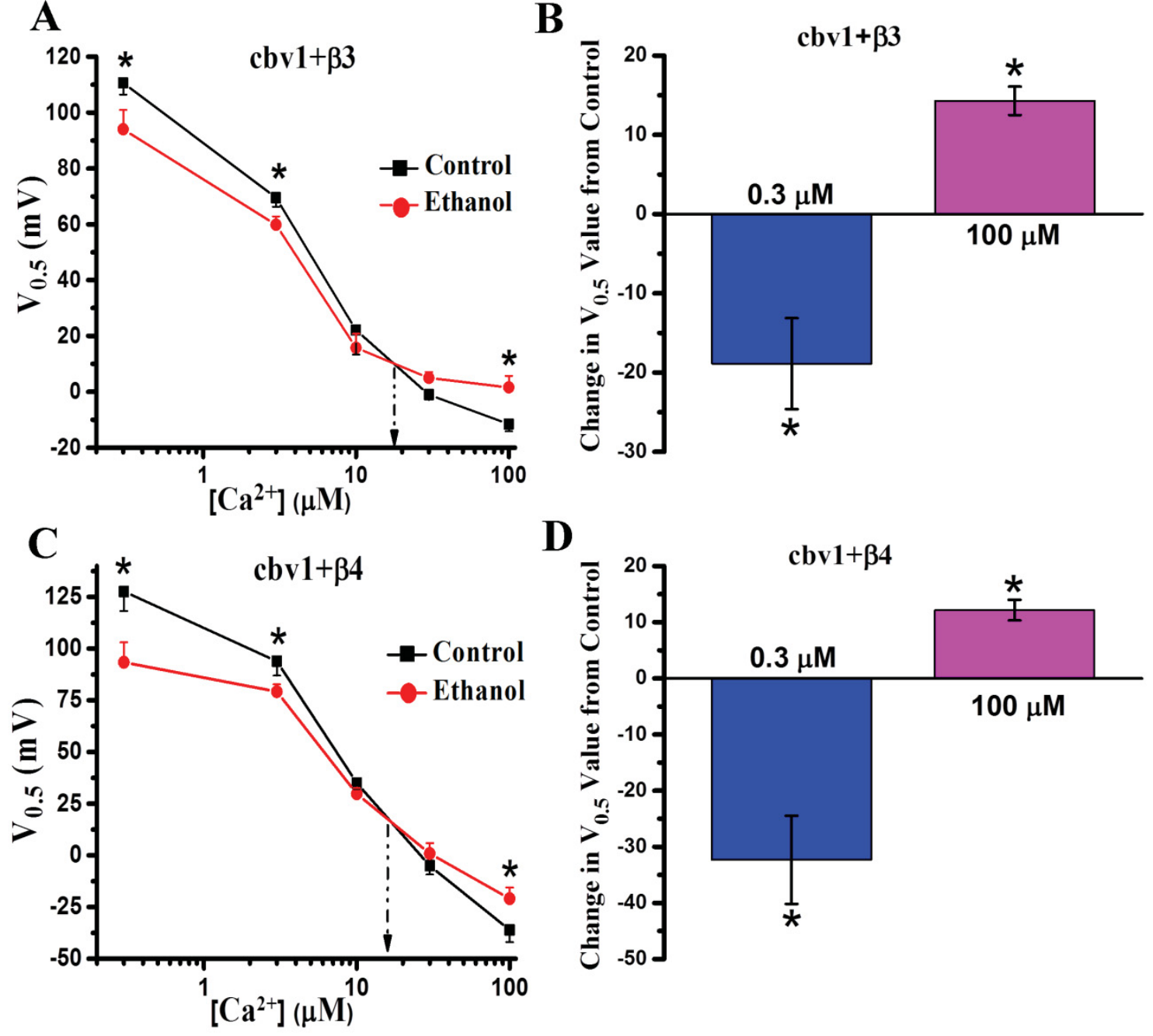

D

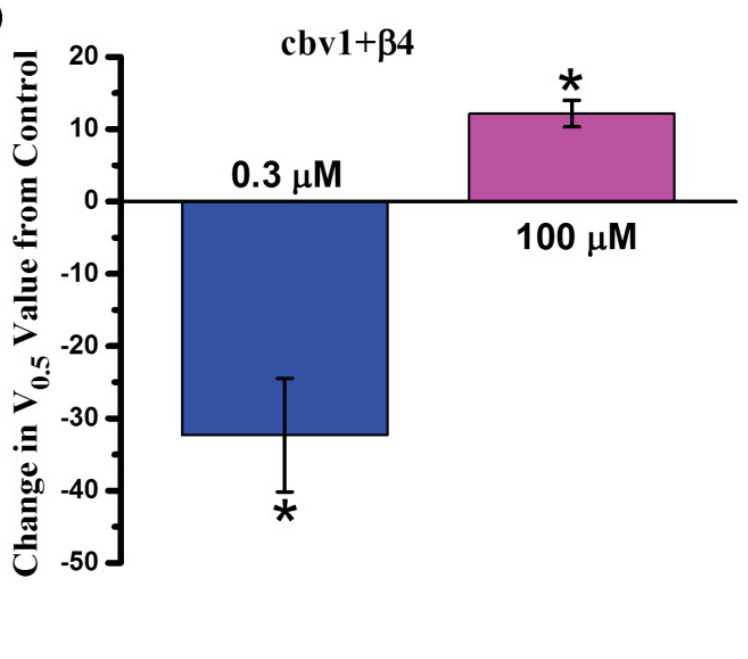

Figure 5-6. Ethanol responses of $\beta 3$ - or $\beta 4-c o n t a i n i n g ~ B K$ currents mimic those of homomeric BK (cbv1) channels

Notes: $\mathrm{V}_{0.5}$ vs. $\left[\mathrm{Ca}^{2+}{ }_{\mathrm{i}}\right]$ plot showing that the activation-to-inhibition crossover for ethanol effect on cbv $1+\beta 3(\mathrm{~A})$ and cbv $1+\beta 4$ channel complexes $(\mathrm{C})$ occurs at $\approx 20 \mu \mathrm{M} \mathrm{Ca}^{2+}{ }_{\mathrm{i}}$, as found for homomeric cbv1 exponsed to ethanol (see Figure 4-2A). Bar graphs representing ethanol-induced change in $\mathrm{V}_{0.5}$ values from cbv $1+\beta 3(\mathrm{~B})$ and cbv $1+\beta 4$ channel complexes (D) obtained at $0.3 \mu \mathrm{M}$ and $100 \mu \mathrm{M} \mathrm{Ca}^{2+}{ }_{\mathrm{i}}$. Each data point represents the average of no less than 3 patches, each excised from a different cell. *Different from control $(\mathrm{p}<0.05)$. 

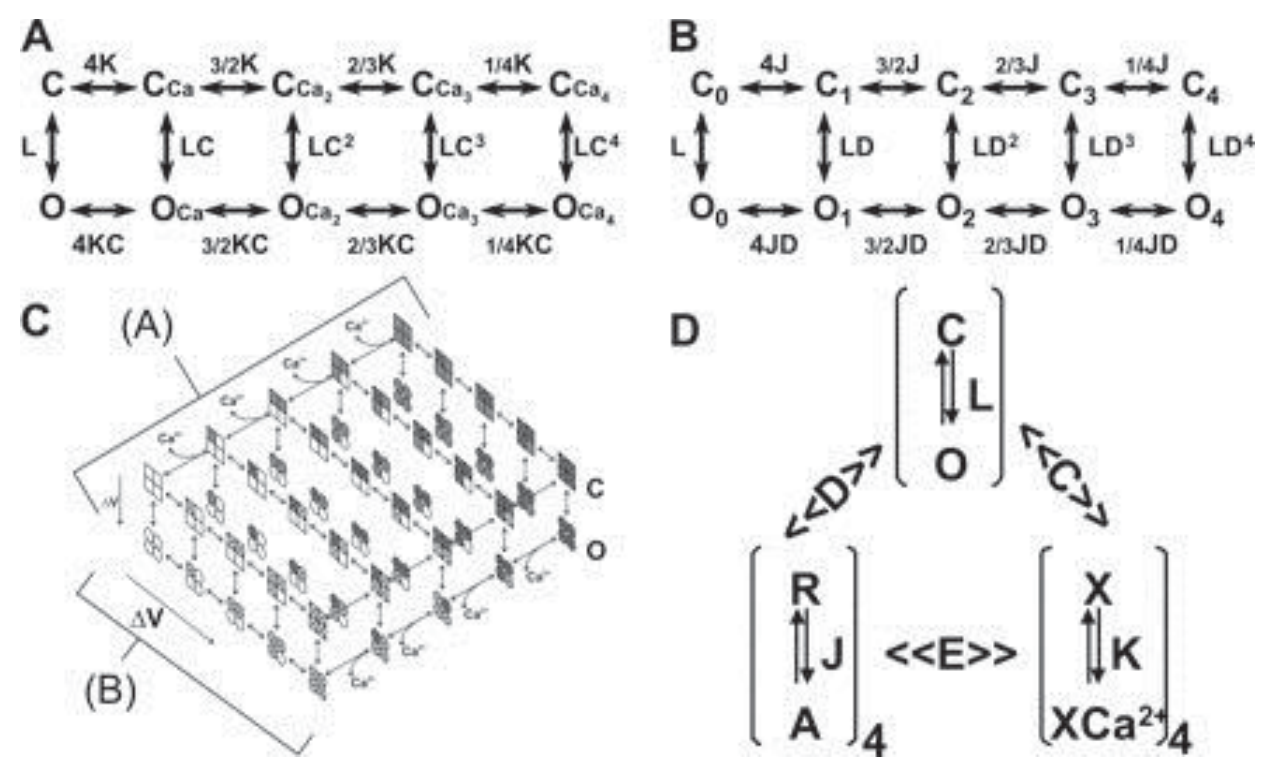

Figure 5-7. Allosteric model for activation of BK channel

Notes: A) 10-state MWC model originated by allosteric activation by $\mathrm{Ca}^{2+}{ }_{\mathrm{i}}$. It is a twotier gating scheme. Top tier includes closed channel states and bottom tier includes open channel states. L represents the equilibrium constant between closed and open states. Once each $\mathrm{Ca}^{2+}{ }_{i}$ binding site is occupied, $\mathrm{L}$ is multiplied by the allosteric factor $\mathrm{C}$. When the channel is open, the $\mathrm{Ca}^{2+}{ }_{i}$ binding equilibrium constant $(\mathrm{K})$ is multiplied by the same allosteric factor C. B) 10-state MWC model representing activation by voltage. Top tier includes closed channel states and bottom tier includes open channel states. L represents the equilibrium constant between closed and open states. Upon each voltage sensor activation, $\mathrm{L}$ is multiplied by the allosteric factor $\mathrm{D}$. When the channel is open, the equilibrium constant for voltage sensor activation $(\mathrm{J})$ is multiplied by the same allosteric factor D. C) two-tired, 50-state model originated by combination of scheme A and scheme B. In this 50-state model, horizontal transitions represent movement of voltage sensors; an " $X$ " within each box (homomer) of the tetrameric channel indicates that the voltage sensor is in its active conformation. Vertical transitions in the scheme represent channel opening. D) A 70-state allosteric model incorporates active interaction between $\mathrm{Ca}^{2+}{ }_{\mathrm{i}}$ binding and sensor activation (allosteric factor E). Voltage sensors can exist in resting $(\mathrm{R})$ and activated $(\mathrm{A})$ states. $\mathrm{Ca}^{2+}{ }_{\mathrm{i}}$ sensors can exist in $\mathrm{Ca}^{2+}$ free $(\mathrm{X})$ or $\mathrm{Ca}^{2+}$ bound state. The function of each structural domain is defined by equilibrium constants for channel opening (L), voltage sensor activation (J), and $\mathrm{Ca}^{2+}$ binding $(\mathrm{K})$. The energy transfer or coupling between different domains is indicated by allosteric factors $(C, D, E)$, which define the ability of a transition in one domain to modulate the equilibrium constant in another domain.

Source: Reprinted with permission from Orio, P and Latorre, R. (2005). Diffrential effects of beta1 and beta2 subunits on BK channel Activity. J Gen Physiol 125(4): 395411. 
In order to address such limitations, 50-state HA model came into existence. In addition to the MWC model assumptions, the 50-state model requires the following: voltage sensors move rapidly in response to changes in membrane voltage regardless of whether the channel is open or closed. Such voltage sensor movement while favoring channel opening, in an allosteric manner, is not required itself for channel opening (Horrigan and Aldrich 1999; Horrigan et al. 1999; Cox and Aldrich 2000; Horrigan and Aldrich 2002) (Scheme B and $\mathbf{C}$ of Figure 5-7).

However, in the models shown above, any possible allosteric coupling among voltage sensor movement, $\mathrm{Ca}^{2+}{ }_{\mathrm{i}}$ binding and channel opening is ignored. The 70 -state allosteric activation model, however, conceives these three processes (voltage sensor activation, $\mathrm{Ca}^{2+}{ }_{\mathrm{i}}$ binding and "intrinsic" channel opening) as independent equilibriums that interact on each other through allosteric coupling, this model being able to explain all characteristics of slo1 channel current phenotypes (Horrigan and Aldrich 1999; Horrigan et al. 1999; Horrigan and Aldrich 2002).

In addition to the parameters included in the 50-state model, the 70-state HA model further takes into account: 1) voltage dependence of voltage sensors; 2) voltage dependence of channel opening; 3) high-affinity $\mathrm{Ca}^{2+}{ }_{i}$ binding and 4) allosteric interaction between $\mathrm{Ca}^{2+}{ }_{i}$ binding and voltage sensor movement (Scheme D of Figure 5-7). For 70-state model, at any voltage and $\mathrm{Ca}^{2+}{ }_{\mathrm{i}}$ concentration, the open probability is given by the Equation 5-1.

$$
P_{o}=\frac{L(1+J D+K C+J K C D E)^{4}}{L(1+J D+K C+J K C D E)^{4}+(1+J K+J K E)^{4}}
$$

Where,

$$
\begin{gathered}
J=\exp \left[\frac{Z_{J} F\left(V-V_{h}(J)\right)}{R T}\right] \\
. K=\frac{\left[C a^{2+}\right]}{K_{d}} \\
L=L_{0} \exp \left(\frac{Z_{L} F V}{R T}\right)
\end{gathered}
$$

Where,

$\mathrm{L}_{0}$, Closed to open conformation equilibrium constant, with no $\mathrm{Ca}^{2+}{ }_{\mathrm{i}}$ bound and $\mathrm{V}=0$ $\mathrm{Z}_{\mathrm{L}}$, the gating charge associate with the channel opening

$\mathrm{V}_{\mathrm{h}}(\mathrm{J})$, represents the voltage at which voltage sensors are half the time active when channel is closed

$\mathrm{Z}_{\mathrm{J}}$, the gating charge associated with each voltage sensor's movement

$\mathrm{K}_{\mathrm{d}}$, is the dissociation constant for single $\mathrm{Ca}^{2+}{ }_{\mathrm{i}}$ binding site, when channel is closed and all voltage sensors are in the resting state

$\mathrm{C}$, allosteric coupling factor between $\mathrm{Ca}^{2+}{ }_{\mathrm{i}}$ binding and channel opening 
$\mathrm{D}$, allosteric coupling factor between voltage sensor activation and channel opening $\mathrm{E}$, allosteric coupling factor between $\mathrm{Ca}^{2+}{ }_{i}$ binding and voltage sensor activation In the equations above, R, T, F have their usual meaning

Summary of various allosteric parameters and their likely corresponding structural domains of BK channel protein are given in Table 5-1.

\subsubsection{Determining Ethanol's Effect on the Movement of Voltage Sensors for Homomeric BK Channels}

Modulation of voltage sensor movement can be best estimated by determining the parameter $\mathrm{V}_{\mathrm{h}}(J)$, which corresponds to the half-activation voltage of the voltage sensors. As previously done by several other groups (Horrigan and Aldrich 1999; Horrigan et al. 1999; Horrigan and Aldrich 2002; Orio and Latorre 2005), this parameter can be obtained by measuring the voltage dependence of activation kinetics in the absence of $\mathrm{Ca}^{2+}$. Thus, macroscopic current were evoked by $100 \mathrm{~ms}$-long $10 \mathrm{mV}$ depolarizing steps from a holding potential of $-80 \mathrm{mV}$, stepping from -150 to $+200 \mathrm{mV}$. Then, the time constant of activation kinetics $\left(\tau_{\text {act }}\right)$ was measured from a built-in function in Clampfit 9 and plotted as function of activation voltage. The voltage at which the $\tau_{\text {act }}-\mathrm{V}$ plot reaches its maximum indicates the $V_{h}(J)$ parameter.

Our results demonstrated that half-activation voltage of the voltage sensors for cbv1 channels occurred at $150 \mathrm{mV}$ (Figure 5-8A and B). This value is in agreement with previously published reports for slo1 channels other than cbv1 (Horrigan and Aldrich 1999; Horrigan et al. 1999; Bao and Cox 2005; Orio and Latorre 2005; Sun et al. 2013). Interestingly, ethanol failed to modulate cbv1 $\mathrm{V}_{\mathrm{h}}(\mathrm{J})$ (Figure 5-8A and B). This result indicates that ethanol at concentration obtained during binge drinking, while altering BK channel gating (Dopico et al. 1998; Liu et al. 2008) does not alter the movement of voltage sensors when the channel is closed.

\subsubsection{Determining Ethanol Effect on the Closed-to-Open Conformational Change on Homomeric Cbv1 Channels}

It has been previously demonstrated that by obtaining the $\mathrm{P}_{\text {open }} v s . \mathrm{V}$ relation of BK channel at very negative potentials and nominal zero $\mathrm{Ca}^{2+}{ }_{i}$ ), it is possible to directly estimate two parameters associated with the closed-to-open conformational change i.e., $\mathrm{Z}_{\mathrm{L}}$ and $\mathrm{L}_{0}$ (Horrigan and Aldrich 1999; Horrigan et al. 1999; Horrigan and Aldrich 2002). When no $\mathrm{Ca}^{2+}{ }_{\mathrm{i}}$ is bound to any $\mathrm{Ca}^{2+}$ binding site of the $\mathrm{BK}$ channel, at far negative voltages, voltage sensors are considered to be at their resting state. 
Table 5-1. Summary of various allosteric model parameters and their likely corresponding structural BK channel domains

\begin{tabular}{cc}
\hline $\begin{array}{c}\text { Allosteric Model } \\
\text { Parameters }\end{array}$ & $\begin{array}{c}\text { Representative } \\
\text { Domain/Interaction }\end{array}$ \\
\hline $\mathrm{L}_{0}$ & Pore \\
$\mathrm{z}_{\mathrm{L}}$ & Pore \\
$\mathrm{V}_{\mathrm{h}}(\mathrm{J})$ & $\mathrm{S} 4 / \mathrm{S} 4-\mathrm{S} 5$ linker \\
$\mathrm{Z}_{\mathrm{J}}$ & $\mathrm{S} 4 / \mathrm{S} 4-\mathrm{S} 5$ linker \\
$\mathrm{D}$ & $\mathrm{S} 4 / \mathrm{S} 4-\mathrm{S} 5$ linker-Pore \\
$\mathrm{K}_{\mathrm{d}}$ & $\mathrm{RCKs}$ \\
$\mathrm{C}$ & $\mathrm{RCKs}-\mathrm{Pore}$ \\
$\mathrm{E}$ & $\mathrm{RCKs}-\mathrm{S} 4 / \mathrm{S} 4-\mathrm{S} 5$ linker \\
\hline
\end{tabular}

Source: Modified with permission from Savalli, N., Pantazis, A., Yusifov, T., Sigg, D. and Olcese, R. (2012). The contribution of RCK domains to human BK channel allosteric activation. J Biol Chem 287(26): 21741-21750. 

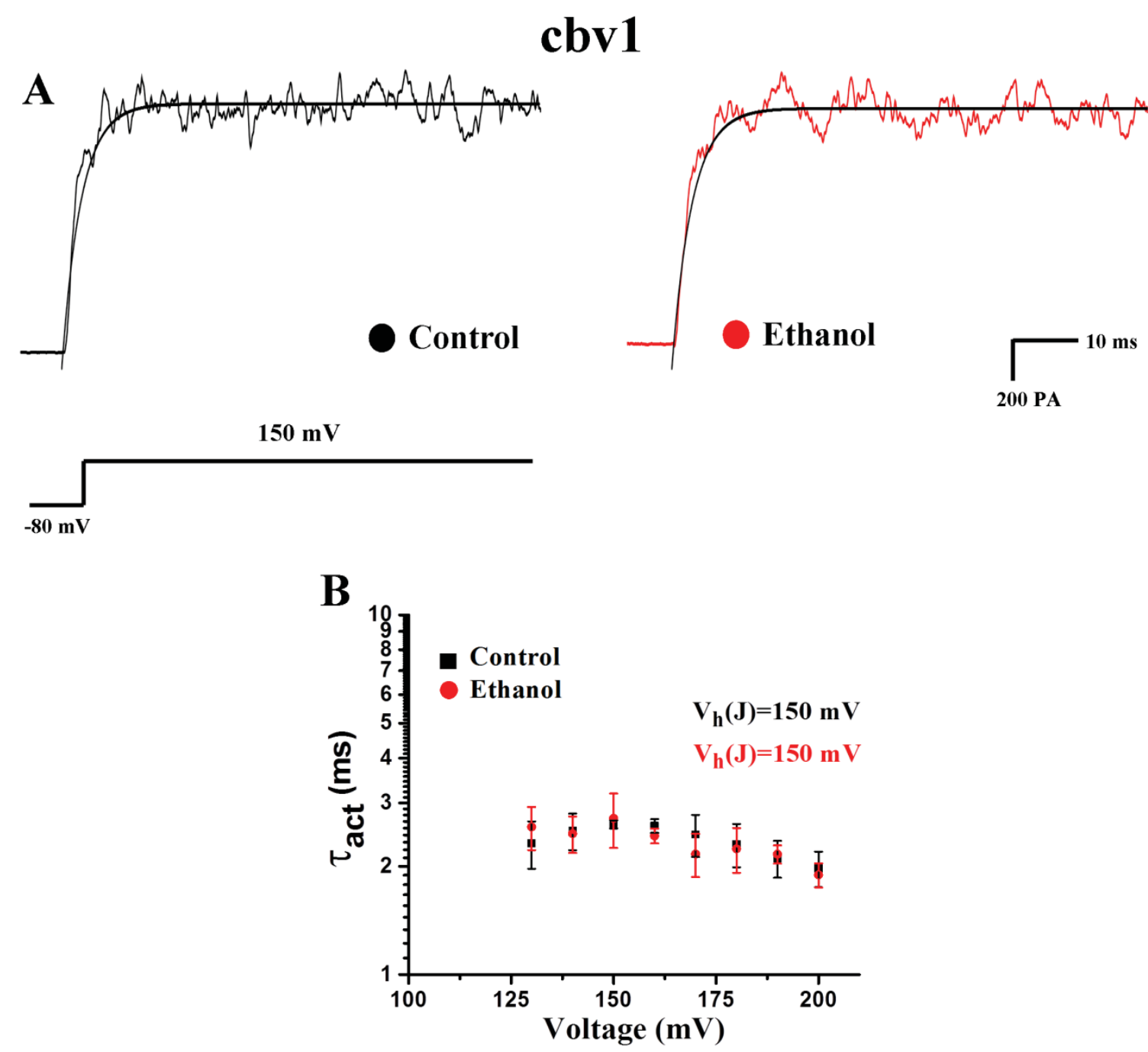

Figure 5-8. Ethanol modulation of voltage dependence of homomeric BK (cbv1) channel activation kinetics in absence of $\mathrm{Ca}^{2+}{ }_{i}$

Notes: A) Macroscopic current traces evoked by a $150 \mathrm{mV}$ pulse after a $-80 \mathrm{mV}$ prepulse in absence (dark trace) and presence (red trace) of $50 \mathrm{mM}$ ethanol. B) Activation time constant $\left(\tau_{\text {act }}\right)$ plotted as a function of membrane step potential. Data demonstrate that the $\tau_{\text {act }}-\mathrm{V}$ relationship reaches a maximum [which indicates $\mathrm{V}_{\mathrm{h}}(\mathrm{J})$ ] at $150 \mathrm{mV}$. Ethanol fails to modulate $\mathrm{V}_{\mathrm{h}}(\mathrm{J})$ in nominal zero $\mathrm{Ca}^{2+}{ }_{i}$. Each data point represents the average of no less than 3 patches, each excised from a different cell. 
Thus the open probability becomes a direct function of voltage and, therefore, Equation 5-1 is reduces to the following equation (i.e., Equation 5-2).

$$
P_{o}=\left[1+\frac{(1+J)^{4}}{L(1+J D)^{4}}\right]^{-1}
$$

Where,

$$
\begin{gathered}
P_{o}=L_{0} \exp ^{\left(z_{L} F V / R T\right)} \\
J=\exp \left(\frac{F\left[V-V_{h}(J)\right]}{R T}\right)
\end{gathered}
$$

According to Equation 5-3, when we hyperpolarize the membrane, a plot of $\log \left(\mathrm{P}_{\text {open }}\right)-\mathrm{V}$ will reach a limiting slope that is less than the maximum slope. This limiting slope reflects the voltage dependence of the closed-to-open conformational change (Horrigan and Aldrich 1999; Horrigan et al. 1999). Therefore, at this specific voltage range, the slope of $\log \left(\mathrm{P}_{\text {open }}\right)-\mathrm{V}$ relation will be solely determined by $\mathrm{z}_{\mathrm{L}}$ while the position of the curve along the vertical axis will be determined only by $\mathrm{L}_{0}$.

For cbv1 channels, the best fit values for parameters associated with closed-toconformational change were: $\mathrm{L}_{0}=2 * 10^{-6}$ and $\mathrm{z}_{\mathrm{L}}=0.4$ (Figure 5-9A and B). These values are in accordance with previously published studies for lo1 channels other than cbv1 (Horrigan and Aldrich 1999; Horrigan and Aldrich 2002; Bao and Cox 2005; Orio and Latorre 2005; Sun et al. 2013). Ethanol, however, failed to alter these parameters $\left(\mathrm{L}_{0}=2 * 10^{-6}\right.$ and $\mathrm{z}_{\mathrm{L}}=0.3$ in presence of $50 \mathrm{mM}$ ethanol) (Figure 5-9A, B and C), indicating that ethanol does not modulate the conformational change associated with closed-to-open state.

\subsubsection{Determining Ethanol's Effect on the Remaining Voltage-Dependent Parameters of Cbv1 Channel Gating}

After establishing that ethanol failed to modify cbv1 $\mathrm{V}_{\mathrm{h}}(\mathrm{J}), \mathrm{L}_{0}$ or $\mathrm{Z}_{\mathrm{L}}$, we next determined ethanol action on $\operatorname{cbv} 1 \mathrm{z}_{\mathrm{J}}$ and $\mathrm{D}$. In order to perform this, we combined $\mathrm{P}_{\text {open }}-\mathrm{V}$ data with $\mathrm{G} / \mathrm{G}_{\max }-\mathrm{V}$ data to obtain $\mathrm{P}_{\text {open }}-\mathrm{V}$ curves, as shown in the figure (Figure 5-10A and B). Then, these curves were fitted with Equation 5-3.

While fitting the data obtained from cbv1 channels, $\mathrm{V}_{\mathrm{h}}(\mathrm{J}), \mathrm{L}_{0}, \mathrm{z}_{\mathrm{L}}$ values were constrained to the following values for control vs. ethanol data: $\mathrm{V}_{\mathrm{h}}(\mathrm{J})=150 \mathrm{mV}$ vs 150 $\mathrm{mV}, \mathrm{L}_{0}=2 * 10^{-6} v s .2 * 10^{-6}, \mathrm{Z}_{\mathrm{L}}=0.4 v s .0 .3$. $\mathrm{D}$ and $\mathrm{z}_{\mathrm{J}}$ were allowed to vary freely. Our fitting results show that for cbv1 channels in absence of ethanol, $\mathrm{D}=19.1$ and $\mathrm{z}_{\mathrm{J}}=0.6$

(Figure 5-10A). These results are approximately similar to the values obtained for slo1 channels other than cbv1 by different groups (Horrigan and Aldrich 1999; Cox and Aldrich 2000; Horrigan and Aldrich 2002; Bao and Cox 2005; Orio and Latorre 2005).

Ethanol, however, did not significantly change these values from control data. 
$\mathbf{A}$

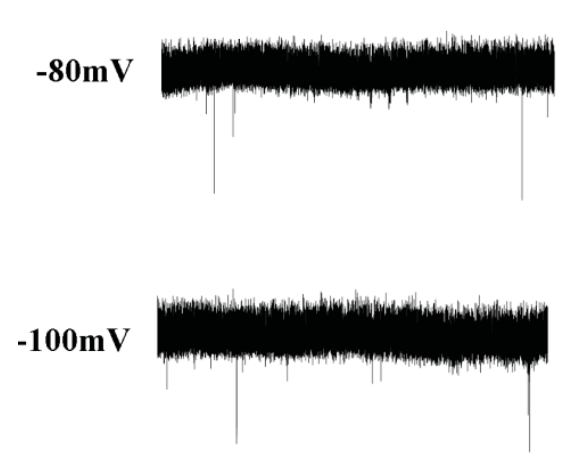

B

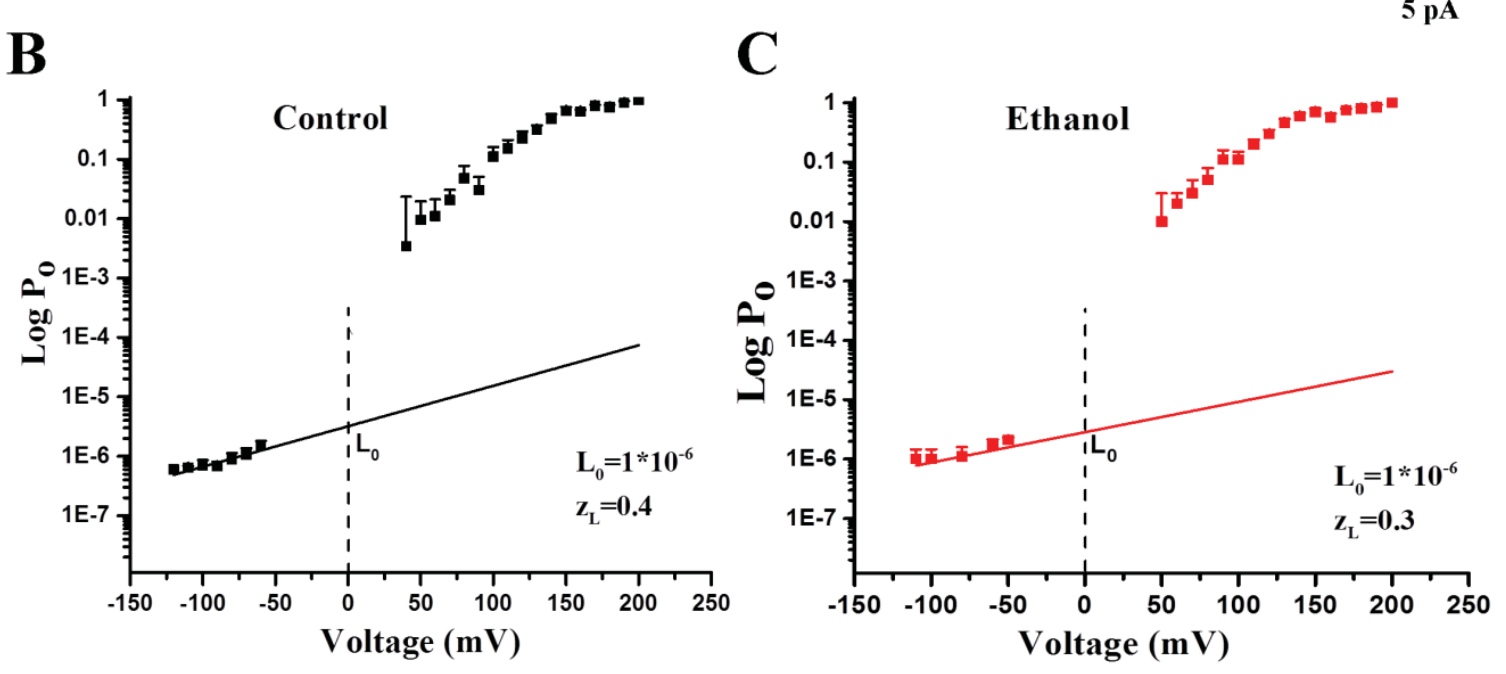

\section{cbv1}
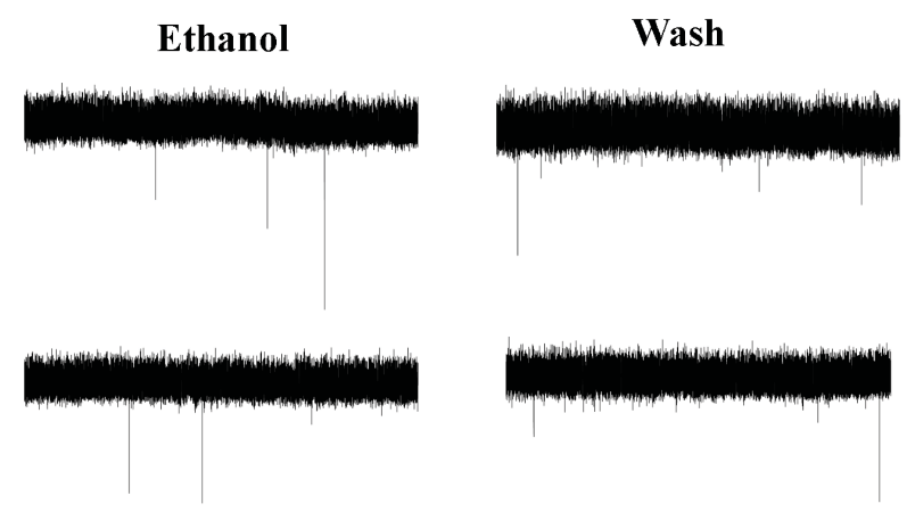

$0.5 \mathrm{~s}$

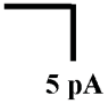

Figure 5-9. Ethanol does not alter the parameters associated with closed-to-open conformational change and voltage dependence of cbv1 channels

Notes: A) Representative unitary current recordings evoked from I/O patches in nominal zero $\mathrm{Ca}^{2+}$; with membrane patches held at the indicated voltages. Recordings were obtained before (left trace), during (middle trace) and immediately after (right trace) patch exposure to $50 \mathrm{mM}$ ethanol. Arrows indicate the baseline (all channels closed). B, C) Mean $\log \mathrm{P}_{0}-\mathrm{V}$ relations over a wide range of voltages in presence and absence of 50 $\mathrm{mM}$ ethanol. Below $+60 \mathrm{mV}$, data points were obtained from unitary current recordings; above $+60 \mathrm{mV}$, data points were obtained from macroscopic current recordings obtained at nominal zero $\mathrm{Ca}^{2+}{ }_{i}$. B) and C) $\mathrm{P}_{0}-\mathrm{V}$ data at far negative voltages were fitted with the Equation 5-3 to determine the values of $\mathrm{L}_{0}$ and $\mathrm{z}_{\mathrm{L}}$. Best fit parameters are listed in the corresponding graphs. Each data point represents the average of no less than 3 patches, each excised from a different cell. 


\section{cbv1}

A

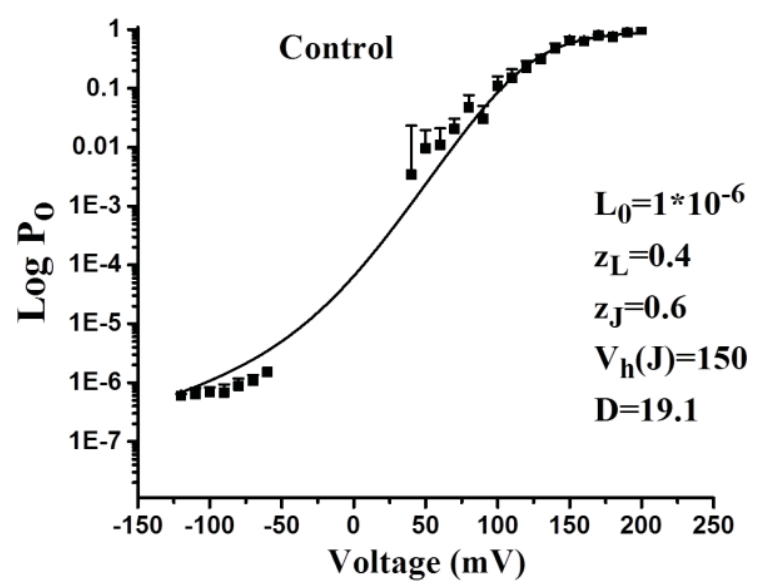

B

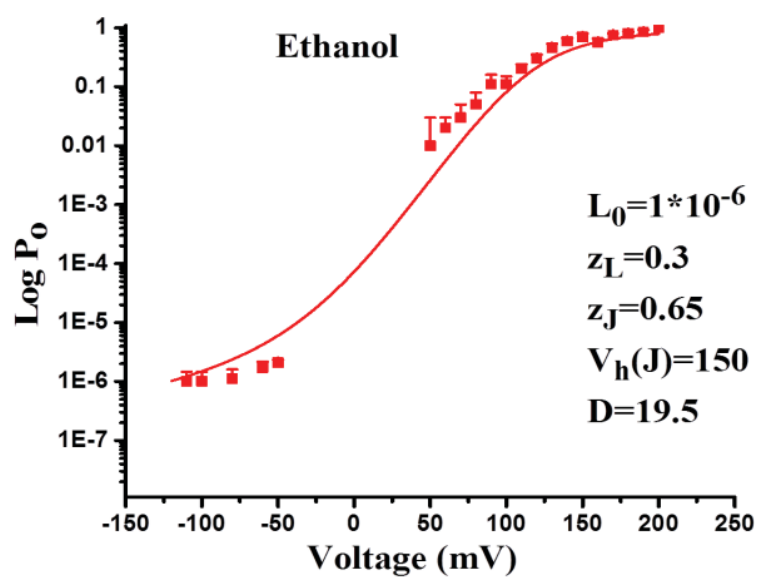

Figure 5-10. Ethanol does not alter the voltage-dependent parameters of cbv1 channels

Notes: A) and B) Po-V data over a wide range of voltages were fitted with Equation 5-2 after restricting $\mathrm{V}_{\mathrm{h}}(\mathrm{J})$, L0 and $\mathrm{z}_{\mathrm{L}}$, to determine the values of $\mathrm{D}$ and $\mathrm{z}_{\mathrm{J}}$. Best fit parameters are listed in the corresponding graphs. Each data point represents the average of no less than 3 patches, each excised from a different cell. 
The values obtained were, $\mathrm{D}=19.5$ and $\mathrm{z}_{\mathrm{J}}=0.65$ in presence of $50 \mathrm{mM}$ ethanol

(Figure 5-10B). These results from homomeric cbv1 channels indicate that ethanol does not alter either the voltage dependence of the voltage sensors or the coupling factor between voltage sensor activation and channel opening.

\subsubsection{Determining Ethanol Effect on Homomeric BK Channel's $\mathrm{Ca}^{2+}{ }_{\mathrm{i}}$ Binding Affinity}

Once we evaluated whether ethanol modulated the voltage sensitivity of the BK channel in the absence of $\mathrm{Ca}^{2+}{ }_{i}$, we next studied ethanol action on various 70 -state HA model parameters as the $\mathrm{Ca}^{2+}{ }_{i}$ concentration was increased. In order to do this, we used $\mathrm{G} / \mathrm{G}_{\max }-\mathrm{V}$ relations obtained at various $\mathrm{Ca}^{2+}{ }_{i}$ concentrations from nominal zero- $100 \mu \mathrm{M}$ levels from cbv1 channels in presence and absence of $50 \mathrm{mM}$ ethanol. $\mathrm{G} / \mathrm{G}_{\max }-\mathrm{V}$ relations were fitted with Equation 5-1 by minimizing least-squares with a mixture of MarquardtLevenverg and Simplex iterations using Origin Pro 8.5 software. Several fits were obtained with different sets of initial parameters and the fits with lower chi-square values were chosen.

Our best fit results obtained for cbv1 channels in absence of ethanol rendered $K_{d}=6.93, C=19.3$ and $E=2.11$ (Figure 5-11A). These values are in reasonable agreement with previously obtained best fit values for slo1 channels other than cbv1 (Horrigan and Aldrich 1999; Horrigan and Aldrich 2002; Orio and Latorre 2005). Interestingly, ethanol increased the $\mathrm{Ca}^{2+}{ }_{\mathrm{i}}$ binding affinity by $\sim 60 \%$, which is evident from decrease in $\mathrm{K}_{\mathrm{d}}$ from 6.93 to 2.86 and (Figure 5-11B). Collectively, all our fitting results indicate that most of ethanol's effect on homomeric BK (cbv1) channels is due to drug-induced increase in $\mathrm{Ca}^{2+}{ }_{\mathrm{i}}$ binding affinity thus leading to increased channel activity.

\subsubsection{Determining Ethanol Effect on BK Channel Voltage Sensor Movement in Presence of $\beta 1$ Subunits}

Our results showed that the half-activation voltage for voltage sensors $\mathrm{V}_{\mathrm{h}}(\mathrm{J})$ for cbv $1+\beta 1$ channels was $100 \mathrm{mV}$ (Figure 5-12A and B). This value is in reasonable agreement with previously published reports for slo1 other than cbv1 when co-expressed with $\beta 1$ subunits (Bao and Cox 2005; Orio and Latorre 2005; Sun et al. 2013). Ethanol failed to modulate this parameter for cbv1+ $\beta 1$ channels (Figure 5-12A and B), indicating that ethanol does not alter voltage sensor's movement of the $\mathrm{Ca}^{2+}{ }^{-}$-unliganded, closed channel. 


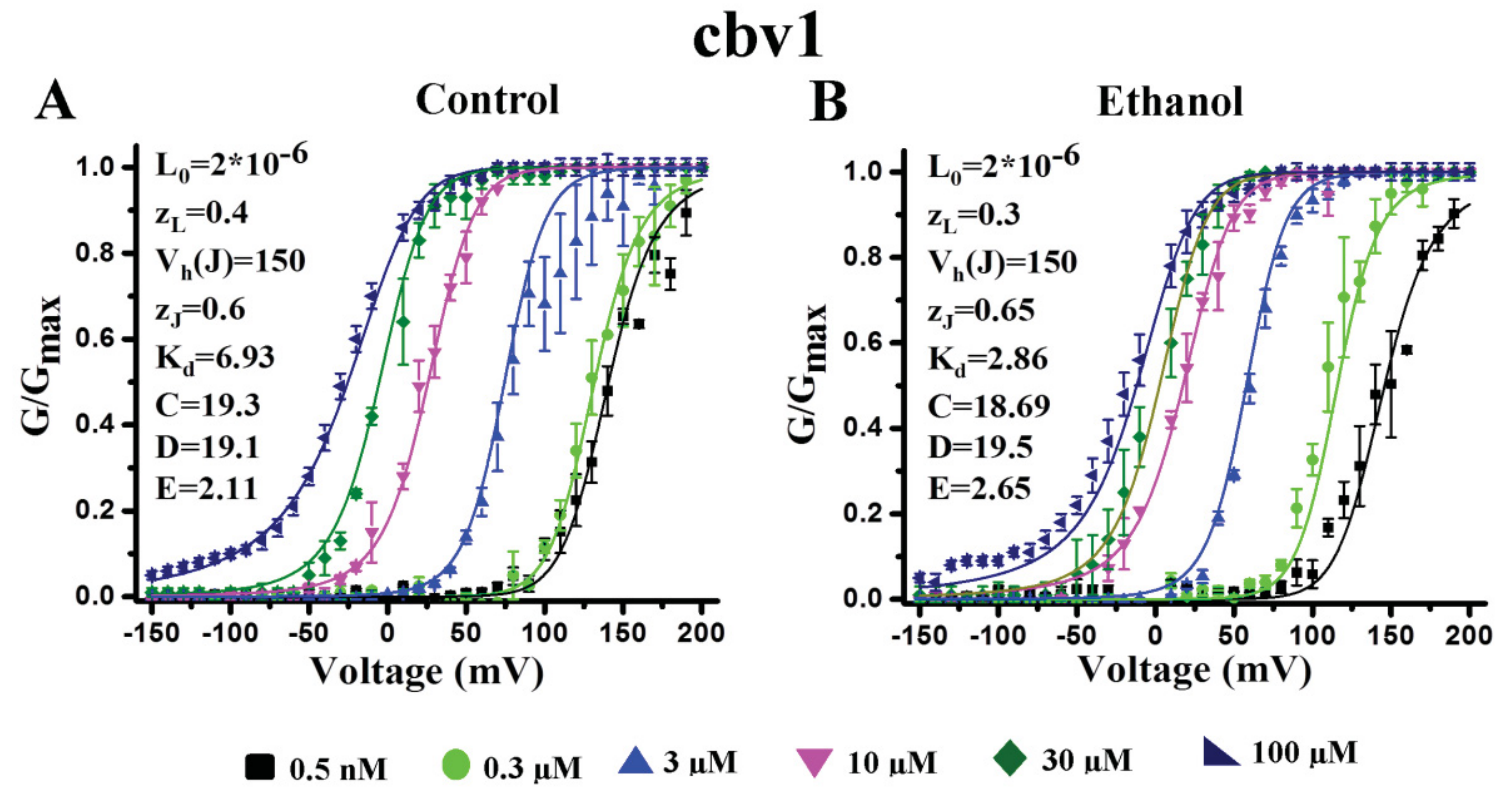

Figure 5-11. Ethanol modulates the parameters associated with $\mathrm{Ca}^{2+}{ }_{\mathrm{i}}$ binding affinity of cbv1 channels

Notes: Averaged $\mathrm{G} / \mathrm{G}_{\max }-\mathrm{V}$ curves obtained over a wide range of $\mathrm{Ca}^{2+}{ }_{\mathrm{i}}$ in absence (A) and presence (B) of $50 \mathrm{mM}$ ethanol. $\mathrm{G} / \mathrm{G}_{\max }-\mathrm{V}$ plots were fitted with Equation 5-1. For curves in panel $A, \mathrm{~L}_{0}, \mathrm{z}_{\mathrm{L}}, \mathrm{V}_{\mathrm{h}}(\mathrm{J}), \mathrm{z}_{\mathrm{J}}$, D were fixed to $2^{*} 10^{-6}, 0.4,150,0.6$, and 19.1, respectively while $\mathrm{K}_{\mathrm{d}}, \mathrm{C}, \mathrm{D}$ and $\mathrm{E}$ were allowed to vary. For $\mathrm{G}-\mathrm{V}$ curves in panel $\mathrm{B}, \mathrm{L}_{0}$, $\mathrm{Z}_{\mathrm{L}}, \mathrm{V}_{\mathrm{h}}(\mathrm{J}), \mathrm{z}_{\mathrm{J}}$, D were fixed to $2^{*} 10^{-6}, 0.3,150,0.65$, and 19.5 , respectively while $\mathrm{K}_{\mathrm{d}}, \mathrm{C}, \mathrm{D}$ and $\mathrm{E}$ were allowed to vary. Best fit parameters are shown to the left of the corresponding plots. Each data point represents the average of no less than 3 patches, each excised from a different cell. 


\section{$\operatorname{cbv} 1+\beta 1$}
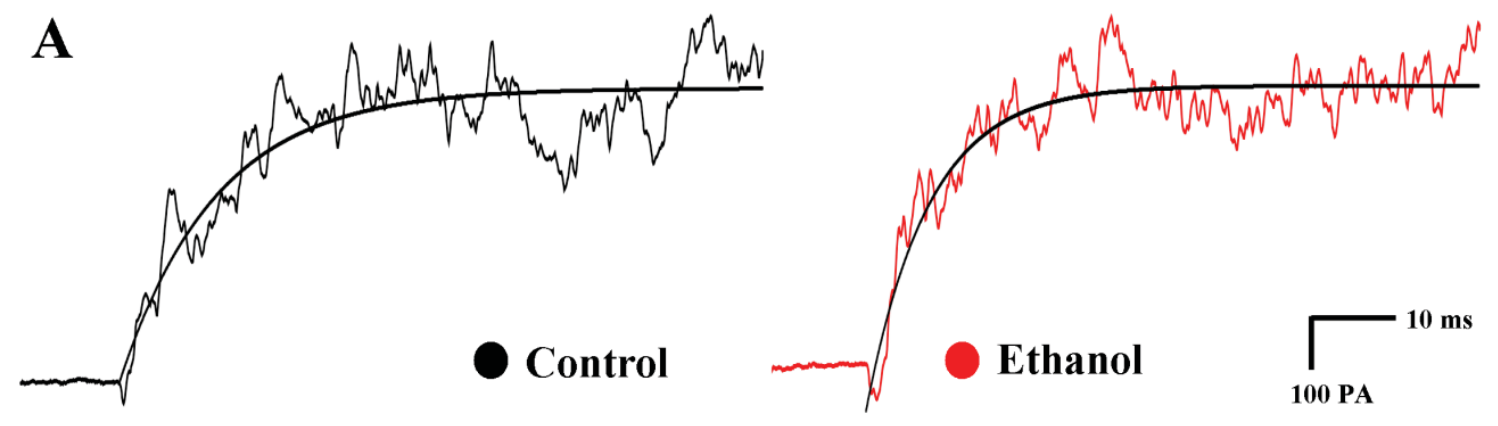

$150 \mathrm{mV}$
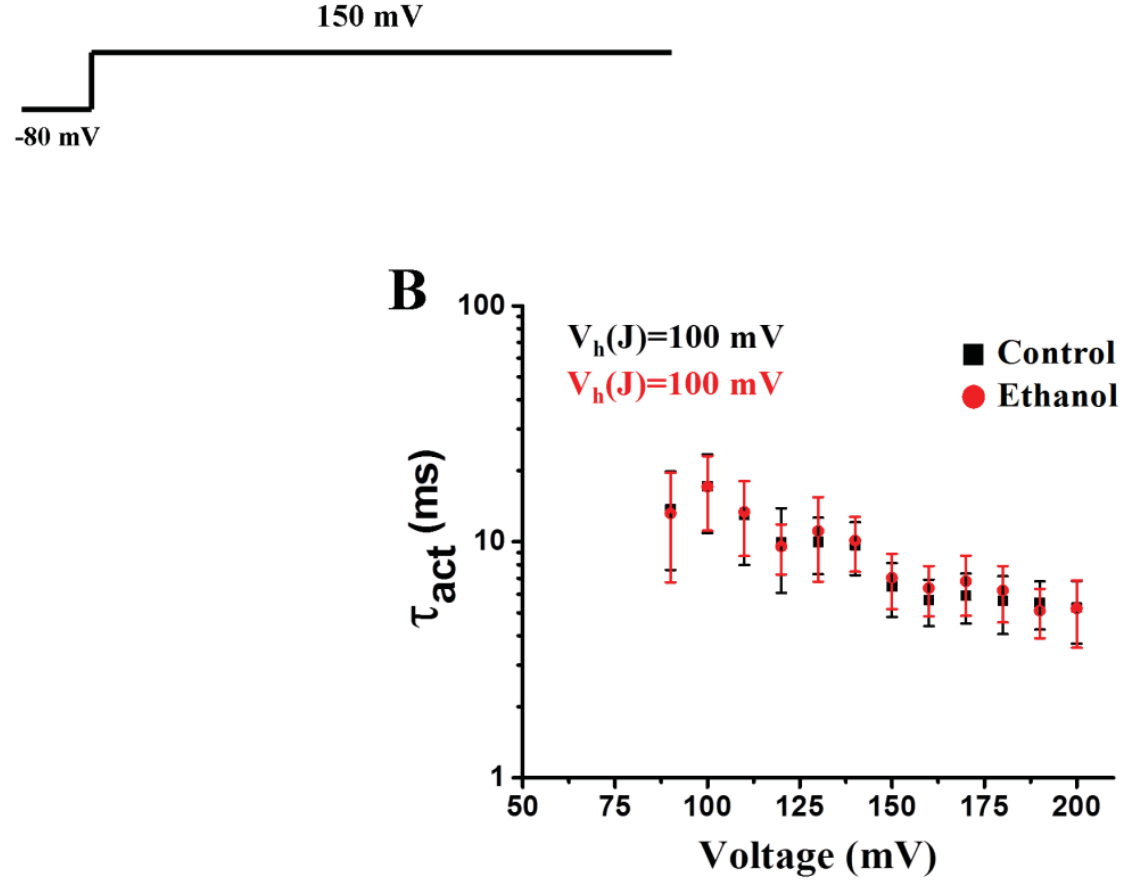

Figure 5-12. Ethanol modulation of voltage-dependent activation kinetics from $\beta 1$ containing $\mathrm{BK}$ channels in the absence of $\mathrm{Ca}^{2+}{ }_{i}$

Notes: A) Macroscopic current traces evoked by a $150 \mathrm{mV}$ pulse after a $-80 \mathrm{mV}$ prepulse in absence (dark trace) or presence (red trace) of $50 \mathrm{mM}$ ethanol. B) Activation time constant $\left(\tau_{\text {act }}\right)$ plotted as a function of membrane potential. Data demonstrate that the $\tau_{\text {act }}-\mathrm{V}$ relationship reaches a maximum [which indicates $\mathrm{V}_{\mathrm{h}}(\mathrm{J})$ ] at $100 \mathrm{mV}$. Ethanol fails to modulate $\mathrm{V}_{\mathrm{h}}(\mathrm{J})$ in nominal zero $\mathrm{Ca}^{2+}{ }_{\mathrm{i}}$. Each data point represents the average of no less than 3 patches, each excised from a different cell. 


\subsubsection{Determining Ethanol Effect on the Closed-to-Open Conformational Change

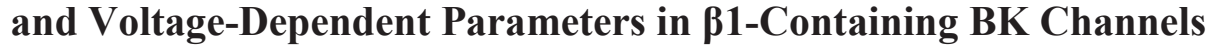

As encountered by several groups (Bao and Cox 2005; Orio and Latorre 2005; Sun et al. 2013), we could not estimate the parameters associated with the closed-to-open equilibrium conformation, i.e., $\mathrm{L}_{0}$ and $\mathrm{z}_{\mathrm{L}}$ for slo1(cbv1 in our case) $+\beta 1$ channels by fitting $P_{0}-V$ data with Equation 5-3. This impossibility results from the fact that, in contrast from slo1 (cbv1) data, $\log \left(\mathrm{P}_{\text {open }}\right)-\mathrm{V}$ plots from cbv $1+\beta 1$ channels do not reach the limiting slope.

Therefore, we determined the effect of ethanol on parameters associated with closed-to-open conformation change $\left(\mathrm{L}_{0}, \mathrm{z}_{\mathrm{L}}\right)$ and the remaining voltage-dependent parameters $(\mathrm{z}, \mathrm{D})$ together for cbv $1+\beta 1$ channels at once. In order to perform this, we combined $P_{\text {open }}-V$ data with $\mathrm{G} / \mathrm{G}_{\max }-\mathrm{V}$ data to obtain $\mathrm{P}_{\text {open }}-\mathrm{V}$ curves (Figure 5-13A, B and C). Then, these $P_{\text {open }}-\mathrm{V}$ data were fitted with Equation 5-2. Thus, $\mathrm{V}_{\mathrm{h}}(J)$ was constrained to the following values for control vs. ethanol data: $\mathrm{V}_{\mathrm{h}}(J)=100 v s$. 100. $\mathrm{L}_{0}, \mathrm{z}_{\mathrm{L}}, \mathrm{D}$ and $\mathrm{z}_{\mathrm{J}}$ were allowed to vary freely. Our fitting results obtained for cbv $1+\beta 1$ channels in absence of ethanol resulted in $\mathrm{L}_{0}=1 * 10^{-7}, \mathrm{z}_{\mathrm{L}}=0.4 \mathrm{D}=37$ and $\mathrm{zJ}_{\mathrm{J}}=0.6$ (Figure 5-13B). These values reasonably match the values obtained by different groups for slo1 other than cbv1 when co-expressed with $\beta 1$ (Cox and Aldrich 2000; Bao and Cox 2005; Orio and Latorre 2005; Sun et al. 2013). Interestingly, ethanol failed to modify any of these parameters: $\mathrm{L}_{0}=1 * 10^{-7}, \mathrm{z}_{\mathrm{L}}=0.4 \mathrm{D}=40$ and $\mathrm{z}_{\mathrm{J}}=0.56$ in ethanol presence (Figure 5-13C). Our results indicate that ethanol does not alter 1) the equilibrium constant between closed-to-open conformation $\left.\left(\mathrm{L}_{0}\right) ; 2\right)$ the voltage-dependence of such equilibrium constant $\left.\left(\mathrm{z}_{\mathrm{L}}\right) ; 3\right)$ the voltage dependence of the voltage sensors $\left(\mathrm{z}_{\mathrm{J}}\right)$ or 4$)$ the allosteric interaction (factor D) between voltage sensor activation and channel opening.

\subsubsection{Determining Ethanol Effect on the $\mathrm{Ca}^{2+}{ }_{\mathrm{i}}$ Binding Affinity of $\beta 1$-Containing BK Channels}

In order to measure the effect of ethanol on $\mathrm{Ca}^{2+}{ }_{i}$ binding affinity and related allosteric factors, we used $\mathrm{G} / \mathrm{G}_{\max }-\mathrm{V}$ relations for cbv1 $1+\beta 1$ channels in presence and absence of ethanol at various $\mathrm{Ca}^{2+}{ }_{i}$ concentrations from nominal zero to $100 \mu \mathrm{M}$. Then, $\mathrm{G} / \mathrm{G}_{\max }-\mathrm{V}$ relations were fitted with Equation 5-1. Our best fit values obtained for cbv $1+\beta 1$ channels in absence of ethanol were $K_{d}=15.17, C=24.25$ and $E=6.16$

(Figure 5-14A). These values are in good agreement with previously best fit values obtained for slo1 channels other than cbv1 when co-expressed with $\beta 1$ subunits (Orio and Latorre 2005). Interestingly, ethanol 1) increased the $\mathrm{Ca}^{2+}{ }_{\mathrm{i}}$ binding affinity by $50 \%$, which was evident from a decrease in $\mathrm{K}_{\mathrm{d}}$ from 15.17 to $7.55 ; 2$ ) decreased the allosteric factor $\mathrm{C}$ by $25 \%$, which represents the interaction between $\mathrm{Ca}^{2+}{ }_{i}$ binding and channel opening; and 3) decreased the allosteric interaction between $\mathrm{Ca}^{2+}{ }_{\mathrm{i}}$ binding and voltage sensor activation by $65 \%$, which was evident by a decrease in $\mathrm{E}$ from 6.16 to 2.09 (Figure 5-14B). 

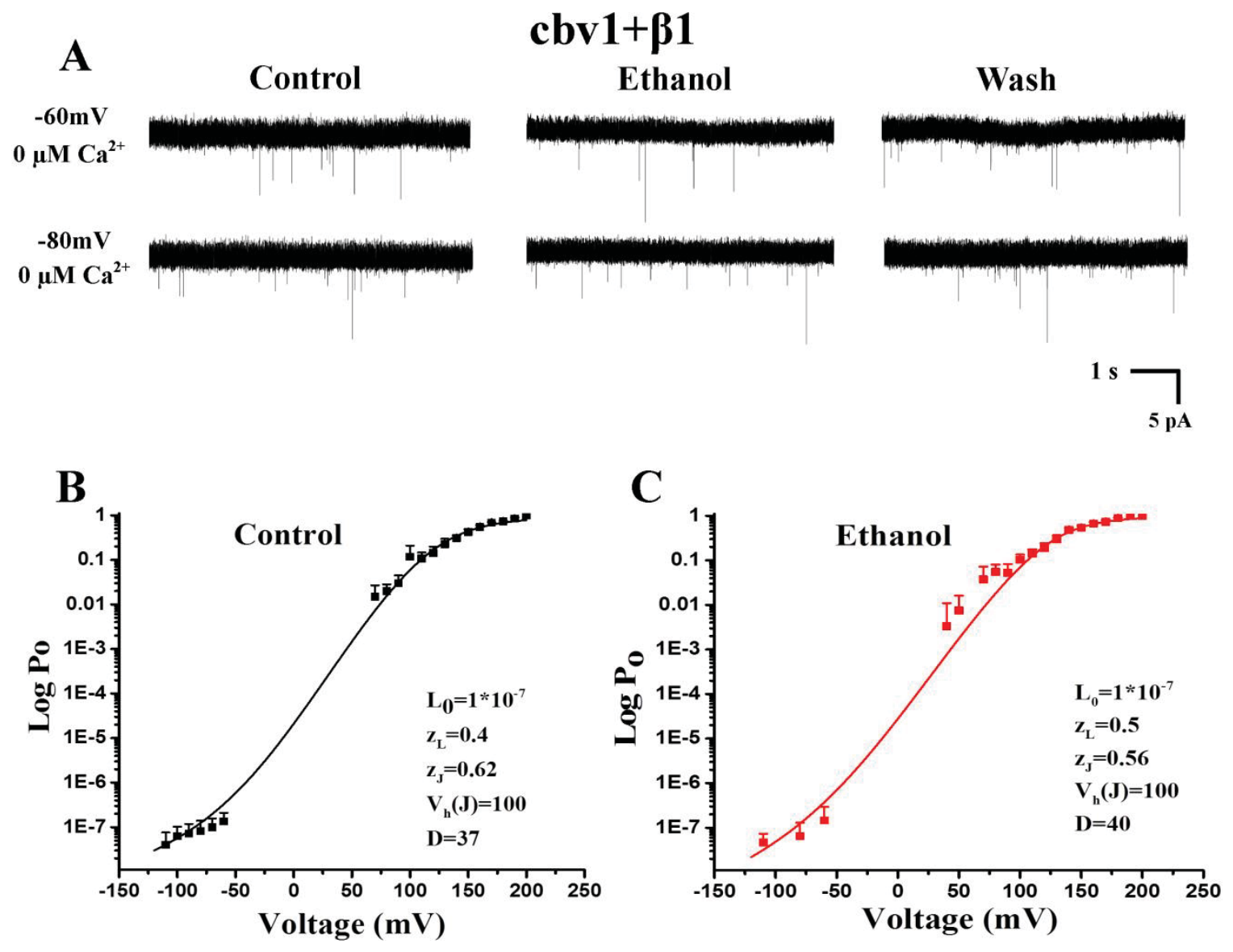

Figure 5-13. Ethanol does not alter the voltage-dependent parameters of cbv1+ $\beta 1$ channels

Notes: A) Representative unitary current recordings from I/O patches evoked in nominal zero $\mathrm{Ca}^{2+}{ }_{\mathrm{i}}$ and within a wide range of membrane potentials. Recordings were obtained before (left trace), during (middle trace) and immediately after (right trace) patch exposure to $50 \mathrm{mM}$ ethanol. Arrows indicate the baseline (all channels closed). B) and C) mean $\log \mathrm{P}_{\mathrm{o}}-\mathrm{V}$ relations in presence and absence of $50 \mathrm{mM}$ ethanol. At voltages less positive than $+60 \mathrm{mV}$, data points were obtained from unitary current recordings; at voltages more positive than $+60 \mathrm{mV}$, data points were obtained from macroscopic current recordings obtained at nominal zero $\mathrm{Ca}^{2+}{ }_{\mathrm{i}} \cdot \mathrm{P}_{\mathrm{o}}-\mathrm{V}$ data over a wide range of voltages were fitted with Equation 5-2 after restricting $\mathrm{V}_{\mathrm{h}}(\mathrm{J})$ to determine the values of $\mathrm{L}_{0}, \mathrm{z}_{\mathrm{L}}, \mathrm{D}$ and $\mathrm{z}_{\mathrm{J}}$. Best fit parameters are listed in the corresponding graphs. Each data point represents the average of no less than 3 patches, each excised from a different cell. 


\section{$\operatorname{cbv1+\beta 1}$}
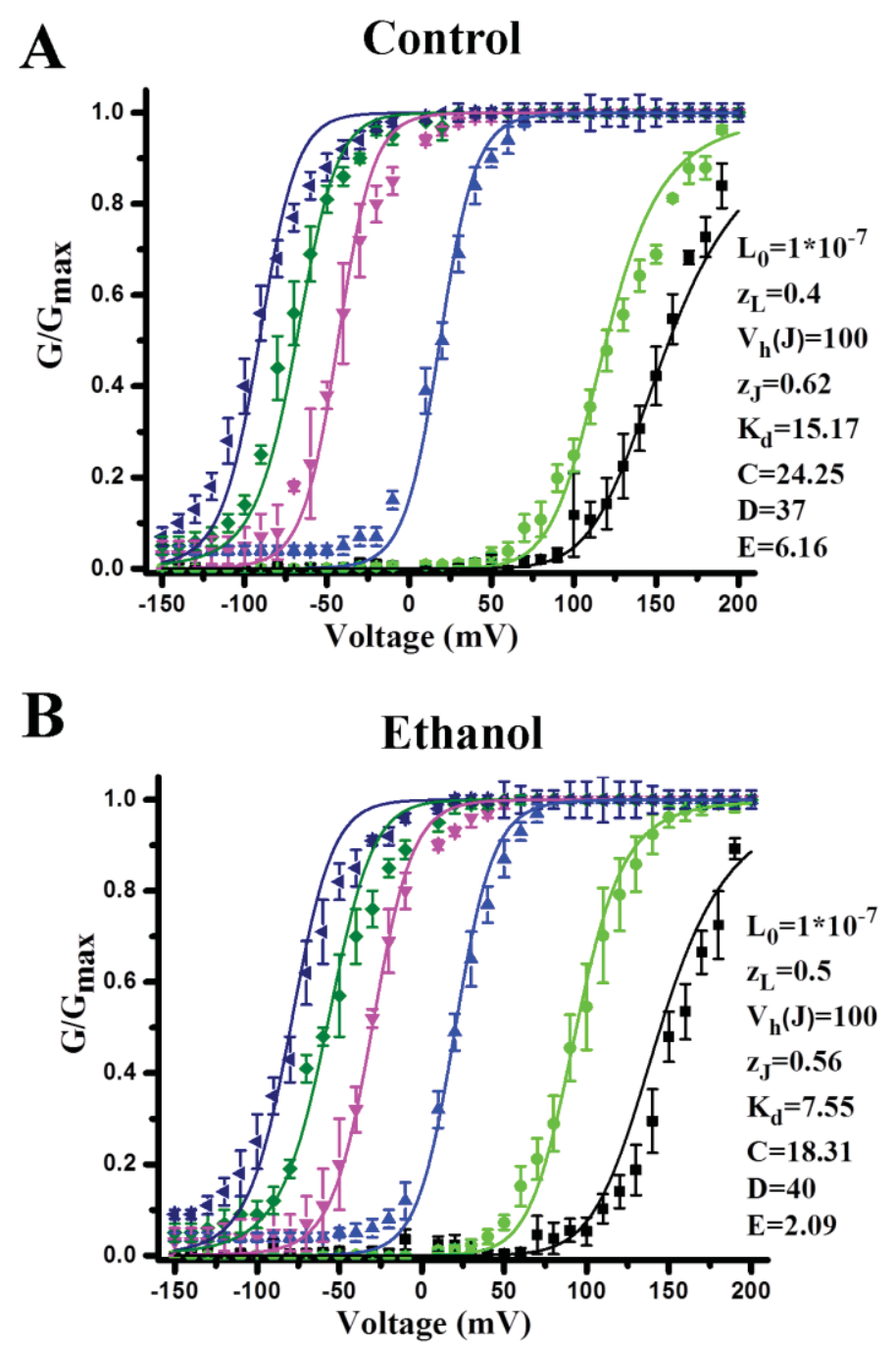

$0.5 \mathrm{nM} \bigcirc 0.3 \mu \mathrm{M} \triangle 3 \mu \mathrm{M} \nabla 10 \mu \mathrm{M}>30 \mu \mathrm{M} \backslash 100 \mu \mathrm{M}$

Figure 5-14. Ethanol modulates the parameters associated with $\mathrm{Ca}^{2+}{ }_{\mathrm{i}}$ binding affinity of cbv1+ $\beta 1$ channels

Notes: Averaged $\mathrm{G} / \mathrm{G}_{\max }-\mathrm{V}$ curves obtained over a wide range of $\mathrm{Ca}^{2+}{ }_{\mathrm{i}}$, in absence (A) and presence (B) of $50 \mathrm{mM}$ ethanol. These plots were fitted with Equation 5-1. For curves in panel $A, \mathrm{~L}_{0}, \mathrm{Z}_{\mathrm{L}}, \mathrm{V}_{\mathrm{h}}(\mathrm{J}), \mathrm{ZJ}_{\mathrm{J}}$, D were fixed to $1^{*} 10^{-7}, 0.4,100,0.6$ and 37

respectively, while $\mathrm{K}_{\mathrm{d}}, \mathrm{C}, \mathrm{D}$ and $\mathrm{E}$ were allowed to vary. For in panel $\mathrm{B}, \mathrm{L}_{0}, \mathrm{z}_{\mathrm{L}}, \mathrm{V}_{\mathrm{h}}(\mathrm{J}), \mathrm{z}_{\mathrm{J}}$ and D were fixed to $1 * 10^{-7}, 0.5,100,0.56$ and 40 , respectively, while $\mathrm{K}_{\mathrm{d}}, \mathrm{C}, \mathrm{D}$ and $\mathrm{E}$ were allowed to vary. Best fit parameters were shown in the corresponding plots. Data demonstrate that ethanol primarily modulates $\mathrm{K}_{\mathrm{d}}, \mathrm{C}$ and $\mathrm{E}$. Each data point represents the average of no less than 3 patches, each excised from a different cell. 
It is noteworthy that ethanol modulation of calcium binding affinity $\left(\mathrm{K}_{\mathrm{d}}\right)$ of cbv $1+\beta 1$ channel complex is similar (i.e., $\sim 50 \%$ increase in $\mathrm{Ca}^{2+}{ }_{i}$ biding affinity) to that of homomeric cbv1 channels (see Table 5-2, Table 5-3 and Figure 5-11A and B). These results indicate that the presence of ethanol does not interfere with $\beta 1$-induced change in $\mathrm{Ca}^{2+}{ }_{i}$ binding affinity of BK channel-forming proteins. Collectively, our fitting results demonstrate that ethanol-induced inhibition of $\beta 1$-containing $\mathrm{BK}$ channel activity is due to a combination of decreased allosteric coupling between 1) $\mathrm{Ca}^{2+}{ }_{i}$ binding and voltage sensor activation and 2) $\mathrm{Ca}^{2+}{ }_{i}$ binding and channel opening. Change in these two gating processes lead to overall decreased channel activity at low micro molar levels of $\mathrm{Ca}^{2+}{ }_{\mathrm{i}}$.

\subsection{Discussion}

In spite of recent advances in our understanding of how ethanol modulates BK channel behavior and thus cell physiology, the structural bases and molecular mechanisms underlying such modulation remain largely unresolved. In particular, while several groups documented that different auxiliary BK $\beta$ subunits condition the channel's final response to ethanol, the use of different BK channel-forming alpha subunits and the largely descriptive electrophysiology approaches used make it impossible to derive any clear conclusion on targeted gating mechanisms and the proteins regions in the BK beta subunits that are responsible for regulating the channel's response to ethanol. In addition to systematically addressing the role of the different BK $\beta$ subunits in ethanol action on BK channels, this study for the first time provides an in depth-analysis of the gating mechanisms that are perturbed by ethanol leading to modification of channel activity. Indeed, we aimed at addressing the following questions, 1) how ethanol distinctly modulates BK channels that only differ in beta subunit type composition and 2) what aspects of channel gating are modified for ethanol to reduce steady-state activity of $\beta 1$ containing BK channels. To do so, we conducted pharmacological, electrophysiological and allosteric modeling approaches on BK macroscopic and single channel currents after expression of recombinant BK subunits in the same expression system, under similar patch-clamp recording conditions and across a wide range of voltage and $\mathrm{Ca}^{2+}{ }_{\mathrm{i}}$ concentrations. Importantly, and unlike all previous studies, we evaluated the ethanol responses of $\mathrm{BK}$ channel complexes formed by different beta subunit types $(\beta 1, \beta 2, \beta 2$ IR, $\beta 3$ or $\beta 4$ ) and the same channel-forming slo1 protein (cbv1). Throughout the study we used an ethanol concentration $(50 \mathrm{mM})$ reached in blood during moderate-to-heavy alcohol intoxication (Liu et al. 2008; Bukiya et al. 2009). Present data demonstrate that 1) ethanol differentially modulates BK currents that result from channel complexes containing different beta subunits; 2 ) ethanol similarly modulates $\beta 1$-, $\beta 2$ - and $\beta 2$-IRcontaining BK channels; 3 ) the inactivation domain of wt $\beta 2$ does not play a major role in ethanol action on cbv1+wt $\beta 2$ channels; 4) ethanol modulation of $\beta 3-, \beta 4$ - containing BK channels matches that of homomeric BK channels. 5) ethanol's major effect on homomeric cbv1 channel gating is to increase the apparent $\mathrm{Ca}^{2+}{ }_{\mathrm{i}}$ binding affinity, which leads to drug-induced potentiation of BK channel steady-state activity (Table 5-2); 6) On the other hand, ethanol-induced inhibition of $\beta 1$-containing BK channels results from a combination of drug-induced gating modifications, as ethanol: 1) increases $\mathrm{Ca}^{2+}{ }_{\mathrm{i}}$ binding affinity of unliganded channel; 2) decreases the allosteric interaction between 
Table 5-2. Summary of best fit parameters obtained for homomeric BK channel in presence or absence of $50 \mathrm{mM}$ ethanol

\begin{tabular}{ccc}
\hline Parameters & Control & $\mathbf{5 0 ~ m M ~ E t h a n o l ~}$ \\
\hline $\mathrm{L}_{0}$ & $2 \times 10^{-6}$ & $2 \times 10^{-6}$ \\
$\mathrm{Z}_{\mathrm{L}}$ & 0.4 & 0.3 \\
$\mathrm{~V}_{\mathrm{h}}(\mathrm{J})$ & 150 & 150 \\
$\mathrm{Z}_{\mathrm{J}}$ & 0.6 & 0.65 \\
$\mathrm{D}$ & 19.1 & 19.5 \\
$\mathrm{~K}_{\mathrm{d}}$ & 6.93 & 2.86 \\
$\mathrm{C}$ & 19.3 & 18.69 \\
$\mathrm{E}$ & 2.11 & 2.65 \\
\hline
\end{tabular}


Table 5-3. Summary of best fit parameters obtained for $\beta 1$-contianing BK channel in presence or absence of $50 \mathrm{mM}$ ethanol

\begin{tabular}{ccc}
\hline Parameters & Control & 50 mM Ethanol \\
\hline $\mathrm{L}_{0}$ & $1 \times 10^{-7}$ & $1 \times 10^{-7}$ \\
$\mathrm{Z}_{\mathrm{L}}$ & 0.4 & 0.5 \\
$\mathrm{~V}_{\mathrm{h}}(\mathrm{J})$ & 100 & 100 \\
$\mathrm{Z}_{\mathrm{J}}$ & 0.65 & 0.56 \\
$\mathrm{D}$ & 37 & 40 \\
$\mathrm{~K}_{\mathrm{d}}$ & 15.77 & 7.55 \\
$\mathrm{C}$ & 24.25 & 18.31 \\
$\mathrm{E}$ & 6.16 & 2.09 \\
\hline
\end{tabular}


$\mathrm{Ca}^{2+}{ }_{i}$ binding and channel opening; and 3) decreases the allosteric interaction between $\mathrm{Ca}^{2+}{ }_{\mathrm{i}}$ binding and voltage sensor activation (Table 5-3). Collectively, these three perturbations of normal gating process lead to decreased channel open probability.

The similar action of ethanol on $\beta 1$ - and $\beta 2$-containing BK channels raises the hypothesis that ethanol action is related to a common mechanism(s) regulated by these two subunits, which is not extended to the other betas $(\beta 3-4)$. Previous data have shown that ethanol action on BK channel-forming proteins is absolutely dependent on $\mathrm{Ca}^{2+}{ }_{\mathrm{i}}$ (Liu et al. 2008), with the drug final effect also being a function of this divalent concentration (Liu et al. 2008; Liu et al. 2013). Consistently, both $\beta 1$ and $\beta 2$ subunits drastically increase the apparent $\mathrm{Ca}^{2+}{ }_{i}$ sensitivity of the channel, an action not shared by $\beta 3$ nor $\beta 4$. Our ethanol data seem to indicate that auxiliary subunit regulation of ethanol action on slo1 channels is unique to $\beta 1$ and $\beta 2$ subunits, which modulate the channel by a common gating perturbation, that is, an increase in the apparent $\mathrm{Ca}^{2+}{ }_{i}$ sensitivity).

These findings raise speculation that the distinct ethanol sensitivity of $\beta 1$ - and $\beta 2$ containing BK channels is due to amino acid residues that are highly conserved in these regulatory proteins. Previous studies have shown a higher conservation (43\%) of residues between $\beta 1$ and $\beta 2$ subunits when compared to the other two regulatory subunit types (i.e., $\beta 1$ vs $\beta 3$ and $\beta 1$ vs $\beta 4$ ) (Behrens et al. 2000; Brenner et al. 2000). Thus, ethanol may directly interact with some of the residues conserved between $\beta 1$ and $\beta 2$ subunits, whether involved in modulating channel's $\mathrm{Ca}^{2+}{ }_{i}$ sensitivity or not, and thus enabling drug-induced inhibition of channel activity at low micro molar levels of $\mathrm{Ca}^{2+}{ }_{\mathrm{i}}$.

Fitting our data to the 70 -state model provide some mechanistic and structural insights on ethanol modulation of $\beta 1$-containing BK channels. In order to interpret these data, we need to first discuss the allosteric interactions between $\mathrm{Ca}^{2+}{ }_{\mathrm{i}}$ and ethanol on homomeric, BK channel-forming cbv1 proteins.

i) Ethanol action on slol channel gating and activating $\mathrm{Ca}^{2+}{ }_{i}$ : Fitting the ethanol data obtained from homomeric BK channel reveals that most of the ethanol's effect on the channel activity is due to modulation of $\mathrm{Ca}^{2+}$-driven gating i.e., increase in the $\mathrm{Ca}^{2+}{ }_{i}$ binding affinity (decrease in $\mathrm{K}_{\mathrm{d}}$ ) without significant modification of voltage-driven gating parameters or the channel intrinsic gating. From an structural point of view, the close spatial location of our newly identified ethanol sensing site in slo1 channels and the channel's high affinity (low $\mu \mathrm{M})$ (Bukiya et al. 2014) $\mathrm{Ca}^{2+}{ }_{\mathrm{i}}$ binding site (see below) suggests that channel conformational changes upon binding of these two ligands $\left(\mathrm{Ca}^{2+}{ }_{\mathrm{i}}\right.$ and ethanol) reciprocally interact to modulate overall channel activity. Indeed, previous data from our lab have demonstrated that if the two high affinity calcium-binding sites are disrupted by nonconserved mutations, the ethanol sensitivity is lost. The channel, however, remains ethanol-activatable as far as $\mathrm{Ca}^{2+}{ }_{i}$ is able to interact with either of the high affinity sites (Liu et al. 2008). Disruption of the RCK1 affinity site uniquely disrupts channel desensitization and eventual decrease in Podriven by higher $\mathrm{Ca}^{2+}{ }_{i}$ levels, the latter process being favored by ethanol (Liu et al. 2008). Interestingly, recent studies (Bukiya et al. 2014) from our lab show 
that, following mutated constructs: mslo1-K361N,R514N, mslo1-R514N, and mslo1-K361N lack sensitivity to ethanol. However, only the R514N-containing constructs demonstrate an altered $\mathrm{Ca}^{2+}{ }_{i}$ sensitivity. Finally, R514 is shifted far away from the ethanol-interaction site when modeled in the " $\mathrm{Ca}^{2+}$-free" state. Thus, R514 seems to be a key structural component in slo1 CTD that communicates ethanol- and $\mathrm{Ca}^{2+}{ }^{2}$-sensing machineries (Bukiya et al. 2014).

Ligand binding sites for ethanol and $\mathrm{Ca}^{2+}{ }_{\mathrm{i}}$ are nearby but do not overlap (Liu et al. 2008; Bukiya et al. 2014), which makes alcohol and $\mathrm{Ca}^{2+}{ }_{i}$ heterotropic ligands of BK channel-forming proteins. The newly identified ethanol sensing site (which involves K361 for hydrogen bonding with the alcohol) in slo1 channels is located adjacent to the RCK1 high affinity $\mathrm{Ca}^{2+}{ }_{\mathrm{i}}$ binding site, which is determined by D362 and D367. Our recent computational modeling data demonstrate that ethanol cannot access the ethanol sensing pocket to hydrogen bond with $\mathrm{K} 361$ in the $\mathrm{Ca}^{2+}{ }_{i}$ free closed channel (Bukiya et al. 2014), which explains the requirement of $\mathrm{Ca}^{2+}{ }_{i}$, but not $\mathrm{Mg}^{2+}$, for ethanol to modulate channel activity (Figure 4-2 and Figure 4-3 in this thesis) (Liu et al. 2008; Liu et al. 2013). While the specific recognition sites for ethanol and $\mathrm{Ca}^{2+}$ do not overlap, they must converge on a common structure that couples gating to channel opening. It has been proposed that eight RCK domains form a "gating ring" (Jiang et al. 2002; Horrigan 2012) for BK channels. This overall design is shared with the two TM-expanding channel, MthK, which is, like slo1, $\mathrm{Ca}^{2+}{ }_{\mathrm{i}^{-}}$-sensitive. Remarkably, our data show that Mthk and slo1 are also ethanol-sensitive (Liu et al. 2013). It is thought that the slo1/Mthk gating ring plays an important role in extracting the free energy from $\mathrm{Ca}^{2+}{ }_{\mathrm{i}}$ binding and thus coupling this ion binding to channel opening. In addition, it has been shown that the "linker" between the S6 transmembrane domain and the first RCK domain (which includes the ethanol binding site) acts as a spring, that couples gating ring expansion secondary to $\mathrm{Ca}^{2+}$ binding with channel opening itself (Jiang et al. 2002; Niu et al. 2004; Ye et al. 2006; Wu et al. 2009; Horrigan 2012; Yuan et al. 2012). Therefore, we advance the notion that upon $\mathrm{Ca}^{2+}$-binding to the BK channel CTD high affinity sites, ethanol is able to modulate slo1 channel activity by interacting with a defined pocket nearby the RCK1 high affinity $\mathrm{Ca}^{2+}{ }^{2}$-binding site, leading to increased affinity of the $\mathrm{Ca}^{2+}{ }_{i}$ and eventually resulting in channel activation.

ii) Ethanol-induced gating modifications in $\beta 1$-containing BK heteromers: Like, homomeric BK channels, fitting the ethanol data obtained from $\beta 1$-containing BK channels reveal that most of the ethanol's effect on the channel activity i.e., facilitation of inhibition, is mainly due to the $\mathrm{Ca}^{2+}$-driven gating but not voltagedriven gating. In presence of $\beta 1$, ethanol leads to perturbation of a two major gating processes, which ultimately leads to decreased channel open probability: 1) decrease in the allosteric interaction between $\mathrm{Ca}^{2+}{ }_{i}$ binding and channel opening (i.e., significant decrease in allosteric factor $\mathrm{C}$ by $25 \%$ ) and 2) decrease in the allosteric interaction between $\mathrm{Ca}^{2+}{ }_{\mathrm{i}}$ binding and voltage sensor activation (i.e., significant decrease in allosteric factor E by 65\%). Collectively, final effect of ethanol leads to a reduced increase in the apparent $\mathrm{Ca}^{2+}{ }_{\mathrm{i}}$ sensitivity introduced 
that is normally introduced by $\beta 1$ subunits, a drug effect that we coined as alcohol's "anti- $\beta 1$ action". At least three structural explanations can be provided for this ethanol's anti- $\beta 1$ action: 1) $\beta 1$ subunit does not contain ethanol sensing site. Rather, newly identified ethanol sensing site (slo1 "activation site") (Bukiya et al. 2014) in the cytosolic tail of the slo1 channel may serve as an "inhibition site", ethanol interaction with this site may disrupt the functional coupling between slo1 and $\beta 1$ subunit 2) $\beta 1$ subunit itself possesses an ethanol recognition/interaction site(s) and thereby causing in inhibition or perturbs the functional coupling between slo1 (cbv1 in our case) and $\beta 1$ subunits upon ethanol binding to such site(s); 3 ) Ethanol may disrupt the lipid environment around the BK channel complex and thereby perturbs the functional coupling between slo 1 and $\beta 1$ subunits. In any case, the final effect of ethanol on $\beta 1$-containing BK channels is to decrease the ability of this regulatory subunit to increase the channel's apparent $\mathrm{Ca}^{2+}{ }_{\mathrm{i}}$-sensitivity.

From a structural point of view, present data may also provide insights on ethanol modulation of specific BK- $\beta 1$ protein domains that participate in basal slo1- $\beta 1$ coupling. Chimeric channel studies between mslo and dslo have demonstrated that slo1 N-end and S0 are both critical for the functional effects of $\beta 1$ (Wallner et al. 1996; Morrow et al. 2006; Lee et al. 2010). Disulfide crosslinking studies shown that N-terminal EC end of S0 is in close proximity of S3 and S4 during resting state and move in concert during voltage sensor activation (Wu et al. 2009; Liu et al. 2010; Wu et al. 2013). Amino acid substitutions in S0 disrupt the voltage-dependent channel activation of BK channels, underscoring S0 interactions with other membrane-spanning domains of the channel VSD (Wallner et al. 1996). Similar disulfide cross-linking studies demonstrate that BK $\beta$ TM1 and TM2 are both packed close to each other at the mouth of the cleft between the VSDs of two adjacent slo1 subunits (Wu et al. 2009; Liu et al. 2010; Wu et al. 2013). Within this cleft, TM1 is close to S1 of one VSD while TM2 is close to S0 of the adjacent VSD (Wu et al. 2009; Liu et al. 2010; Wu et al. 2013). I have recently demonstrated that both TMs of $\beta 1$ subunit are essential to confer the $\beta 1$-induced apparent increase in $\mathrm{Ca}^{2+}{ }_{i}$ sensitivity (Kuntamallappanavar et al. 2014). Thus, the proposed location of both TM1 and TM2 (Wu et al. 2009; Liu et al. 2010; Wu et al. 2013) and our recent findings on functional coupling between slo1 and $\beta 1$ (Kuntamallappanavar et al. 2014) suggest that both TMs of $\beta 1$ subunit and slo1 VSD TMs are functionally coupled. It may be possible that ethanol targets transmembrane domains of $\beta 1$ subunit to disrupt this functional coupling between slo 1 and $\beta 1$ subunit (see previous paragraph), thereby, antagonizing the functional effects of $\beta 1$ subunits. It means in presence of $\beta 1$ subunit, ethanol should allosterically modulate at least some interaction between structural domains that are coupled to gating and channel opening. Previous investigations demonstrated that RCK domains interact with VSD via voltage sensor paddle (i.e., S4 domain) as well as S4-S5 linker and RCKs also interact with PGD via "S6-RCK linker" (Horrigan and Aldrich 2002; Jiang et al. 2002; Hu et al. 2003; Orio and Latorre 2005). Our speculation seems to be consistent with the results obtained from HA model fitting that: in presence of $\beta 1$ subunit, 
ethanol seems to disrupt 1) the active allosteric interaction that involves RCKs and VSD via S4 and S4-S5 linker (i.e., decrease in allosteric factor E) and 2) destabilize the active interaction between RCKs and PGD via S6-RCK linker (i.e., decrease in allosteric factor C), eventually decreasing the channel open probability.

Current data presented and discussed in this section provides evidence of specific regions and gating mechanisms that are targeted by ethanol to modify betal subunitcontaining BK channels. This information may lead to design of new agents that target such region and/or the specific ethanol-sensitive gating processes in order to oppose ethanol-induced modification of physiology via $\beta 1$ subunit-containing BK channels. Future studies might lead to possible identification of specific amino acids in the $\beta 1$ subunit TM2 domain, which can serve for pharmacophore design and drug discovery. 


\section{CHAPTER 6. SPECIFIC BETA1 REGION(S) IS NECESSARY FOR ETHANOL-INDUCED INHIBITION OF BK CHANNELS AND EVENTUAL CEREBRAL ARTERY CONSTRICTION}

\subsection{Overview}

Ethanol at concentrations obtained in circulation during moderate to heavy episodic drinking, such as during binge drinking (30-60 mM) causes cerebral vasoconstriction in many species, including humans. Rodents serve as an excellent model to study ethanol-induced cerebral artery constriction. Using this model, our laboratory demonstrated that ethanol-induced cerebral artery constriction is due to a decrease in STOCs (Spontaneous Transient Outward Currents). In cerebral artery myocytes, STOCs result from the activity of BK channels. Ethanol $(50 \mathrm{mM})$ decreases the $\mathrm{NP}_{\mathrm{o}}$ of vascular myocyte BK channels leading to increase in cerebral artery tone. Functional BK channels are tetramers of pore-forming slo1 subunits. In most of the tissues, slo1 subunits are associated with small accessory subunits $(\beta 1-4)$ that modify the BK current phenotype and pharmacology. BK $\beta 1$ subunit prevails in vascular smooth muscle while $\beta 4$ subunits prevail in neurons. Previous studies and specific aim 2 of this dissertation demonstrated that smooth muscle cell-abundant BK $\beta 1$ subunit enables ethanol-induced BK channel inhibition at physiological $\mathrm{Ca}^{2+}{ }_{\mathrm{i}}$ and voltages. In contrast, neuronally-predominant BK $\beta 4$ subunit does not support this ethanol action (see results of chapter 5) (Liu et al. 2008; Bukiya et al. 2009). In this study, our goal was to understand the molecular basis of this pharmacological difference between these two $\beta$ subunits and determine which region of BK $\beta 1$ subunit is necessary for ethanol-induced inhibition of BK channels and eventual cerebral artery constriction. In order to address these questions, first we constructed a set of $\beta 1 / \beta 4$ chimeras by swapping different domains of $\beta 1$ and $\beta 4$ in such a way that swapped region became progressively smaller. Secondly, we used patch-clamp studies to evaluate the ethanol responses of macroscopic currents mediated by recombinant BK channels that contained the different $\beta 1 / \beta 4$ chimeras. Finally, we transfected cerebral arteries from $K C N M B 1 \mathrm{~K} / \mathrm{O}$ mouse with the different $\beta 1 / \beta 4$ chimeric constructs and determined the impact of the BK $\beta 1$ region(s) that mediates drug modification of channel function on ethanol-mediated vasoconstriction of pressurized cerebral arteries.

\subsection{Results}

\subsection{1. $\quad \beta 1 / \beta 4$ Chimeric Constructs and Their Functional Characterization}

As stated above, the overall strategy was to construct $\beta 1 / \beta 4$ chimeras by swapping different domains of $\beta 1$ and $\beta 4$ subunits in such a way that the swapped region became progressively smaller. The resulting are: $1 . \beta_{1} \mathrm{TMs}_{4}$, which contains $\mathrm{h} \beta 4 \mathrm{TMs}$ on $\mathrm{h} \beta 1$ "background" (i.e., h $\beta 1$ EC loop and IC ends); 2. $\beta_{4} \mathrm{TMs}_{1}$, which contains $\mathrm{h} \beta 1 \mathrm{TMs}$ on h $\beta 4$ "background" (i.e., h $\beta 4$ EC loop and IC ends); 3. $\beta_{4} \mathrm{TM} 1_{1}$, which contains $\mathrm{h} \beta 1 \mathrm{TM} 1$ 
on h $\beta 4$ "background" (i.e., h $\beta 4$ TM2, EC loop and IC ends); 3. $\beta_{4} \mathrm{TM} 2{ }_{1}$, which contains h $\beta 1$ TM2 on h $\beta 4$ "background" (i.e., h $\beta 4$ TM1, EC loop and IC ends); 4. $\beta_{4} \mathrm{~L}_{1}$, which contains EC loop from $\mathrm{h} \beta 1$ on $\mathrm{h} \beta 4$ "background" (i.e., $\mathrm{h} \beta 4$ TMs and IC ends); 5. $\beta_{1} \mathrm{~L}_{4}$, which contains EC loop from $\mathrm{h} \beta 4$ on $\mathrm{h} \beta 1$ "background" (i.e., h $\beta 1 \mathrm{TMs}$ and IC ends) (Figure 6-1). These $\beta 1 / \beta 4$ chimeric constructs have been routinely used in our laboratory, their surface expression and function when co-expressed with cbv1 being confirmed by pharmacological profiling as described elsewhere (Bukiya et al. 2008; Kuntamallappanavar et al. 2014).

\subsubsection{Both Transmembrane Domains of BK- $\beta 1$ Subunits Are Responsible for Ethanol-Induced Inhibition of BK Channels}

We studied ethanol action using cbv $1+\beta 1$ and $\operatorname{cbv} 1+\beta 4$ as positive and negative control, respectively. All the experimental conditions, data acquisition and analysis were similar to those of the experiments described in SA1.

First, we co-expressed $\beta_{1} \mathrm{TMs}_{4}$ subunits with cbv1 channels and explored ethanol action on the resulting channel complexes. Data demonstrate that ethanol increases the activity of these heterooligomeric channels at low $\left[\mathrm{Ca}^{2+}\right]$ i.e., $0.3-10 \mu \mathrm{M}$, which is evident from the significant decrease in $\mathrm{V}_{0.5}$ from the $\mathrm{V}_{0.5^{-}}\left[\mathrm{Ca}^{2+}\right]$ plot. As $\left[\mathrm{Ca}^{2+}\right]$ increased, activation turned to mild inhibition, which was evident from a mild increase in $\mathrm{V}_{0.5}$. Furthermore, ethanol-induced activation-to-inhibition cross-over occurred at $\sim 20 \mu \mathrm{M}$ (Figure 6-2A and B). Thus, ethanol modulated cbv1 $+\beta_{1} \mathrm{TMs}_{4}$ channel activity in a pattern that matched that of homomeric BK channels. These results seem to indicate that regions that constitute the $\beta 1$ background (IC ends and EC loops from $\beta 1$ ) are not critical for ethanol-induced inhibition of $\beta 1$-containing BK channels.

Next, we tested whether TMs could play a critical role in ethanol-induced action on $\beta 1$-containing BK channels. Thus, we co-expressed $\beta_{4} \mathrm{TMs}_{1}$ subunits with cbv 1 channels and explored ethanol action on these channel complexes. Data demonstrate that ethanol increased channel activity at low $\left[\mathrm{Ca}^{2+}\right]$ i.e., $0.3 \mu \mathrm{M}$, which is evident from the significant decrease in $\mathrm{V}_{0.5}$ values in the $\mathrm{V}_{0.5}-\left[\mathrm{Ca}^{2+}\right]$ plot. As $\left[\mathrm{Ca}^{2+}\right]$ increased, activation turned into significant inhibition, which is evident from the increased $\mathrm{V}_{0.5}$. Remarkably, ethanol-induced activation-to-inhibition cross-over occurred at $\sim 2 \mu \mathrm{M}$

(Figure 6-2C and D), which exactly matched that of cbv1+ $\beta 1$ channels. These results seem to indicate that either or both transmembrane domains from $\beta 1$ are critical for $\beta 1$ subunit to facilitate ethanol inhibition of BK channels. This outcome encouraged me to identify whether an individual transmembrane domain (TM1 or TM2) of BK $\beta 1$ could be responsible for this subunit-induced facilitation of ethanol inhibition of BK channel activity. 


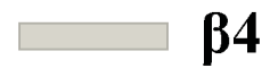

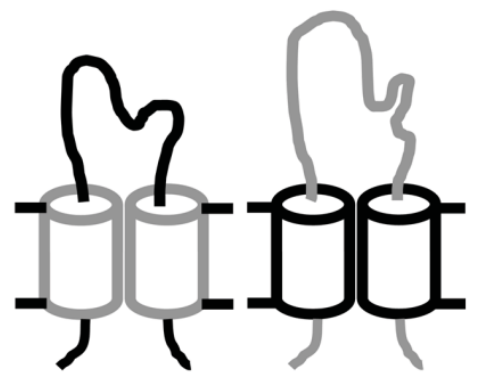

$\beta_{1} \mathrm{TMS}_{4} \quad \beta_{4} \mathrm{TMs}_{1}$

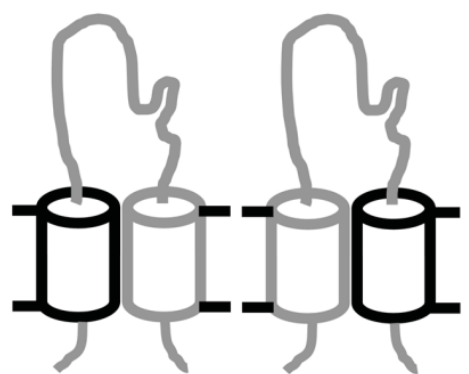

$\beta_{4}$ TM1

$\beta_{4} \mathrm{TM}_{1}$

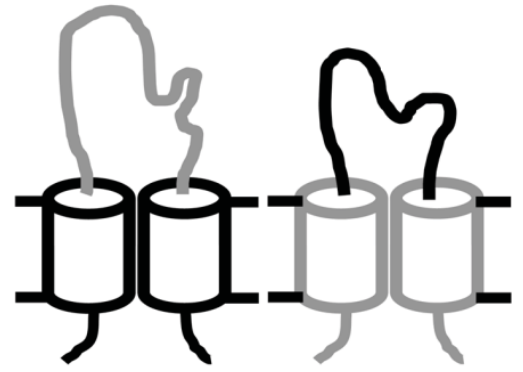

$\beta_{1} L_{4}$

$\beta_{4} L_{1}$

Figure 6-1. Cartoons of $\beta 1 / \beta 4$ chimeras constructed for this study

Notes: Cartoons depicting chimeric constructs that result from swapping different regions between $\beta 1$ and $\beta 4$ subunits. 1. $\beta_{1} \mathrm{TMs}_{4}$, which contains $\mathrm{h} \beta 4 \mathrm{TMs}$ on $\mathrm{h} \beta 1$ "background" (i.e., h $\beta 1$ EC loop and IC ends); 2. $\beta_{4} \mathrm{TMs}_{1}$, which contains $\mathrm{h} \beta 1 \mathrm{TMs}$ on $\mathrm{h} \beta 4$ "background" (i.e., h $\beta 4$ EC loop and IC ends); $3 . \beta_{4} \mathrm{TM}_{1}$, which contains $\mathrm{h} \beta 1 \mathrm{TM} 1$ on h $\beta 4$ "background" (i.e., h $\beta 4$ TM2, EC loop and IC ends); 3. $\beta_{4} \mathrm{TM} 2_{1}$, which contains h $\beta 1$ TM2 on $\mathrm{h} \beta 4$ "background" (i.e., h $\beta 4$ TM1, EC loop and IC ends); 4. $\beta_{4} \mathrm{~L}_{1}$, which contains EC loop from $h \beta 1$ on $h \beta 4$ "background" (i.e., h $\beta 4$ TMs and IC ends); 5. $\beta_{1} \mathrm{~L}_{4}$, which contains EC loop from $\mathrm{h} \beta 4$ on $\mathrm{h} \beta 1$ "background" (i.e., h $\beta 1 \mathrm{TMs}$ and IC ends) Domains from $\beta 1$ are given in black and those of $\beta 4$ are given in grey.

Source: Modified with permission from Kuntamallappanavar G. Toro L. and Dopico A.M. (2014). Both transmembrane domains of BK betal subunits are essential to confer the normal phenotype of betal-containing BK channels. PLoS One 9(10): e109306. 
A

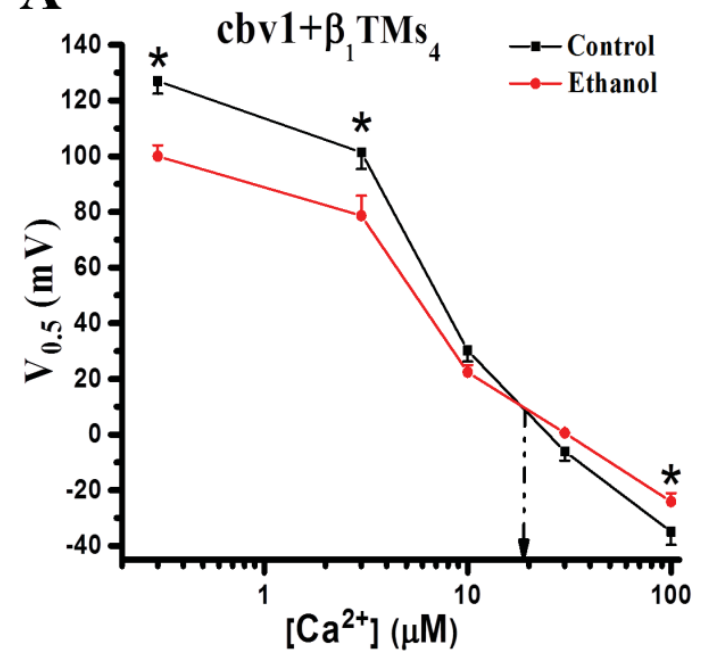

C

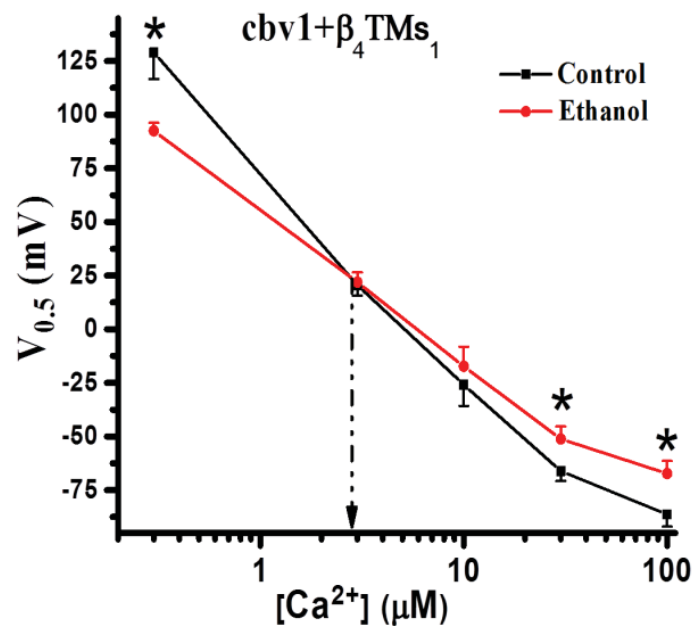

B

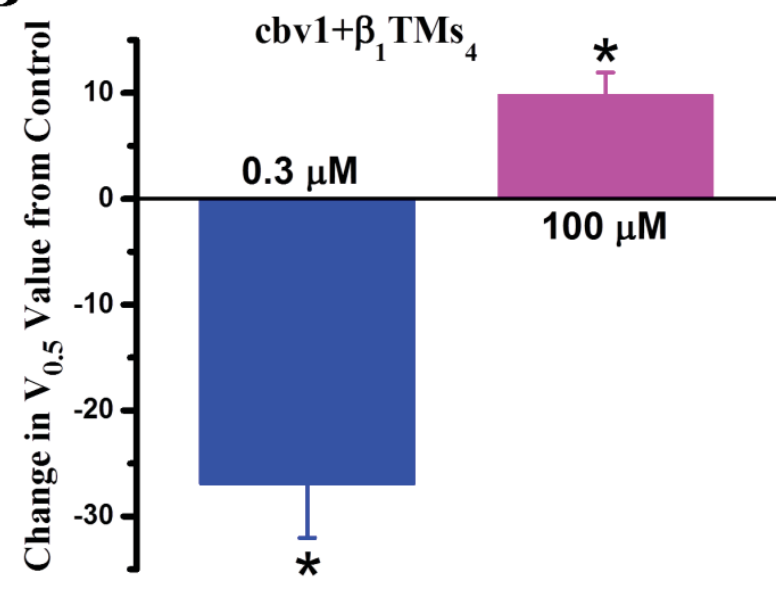

D

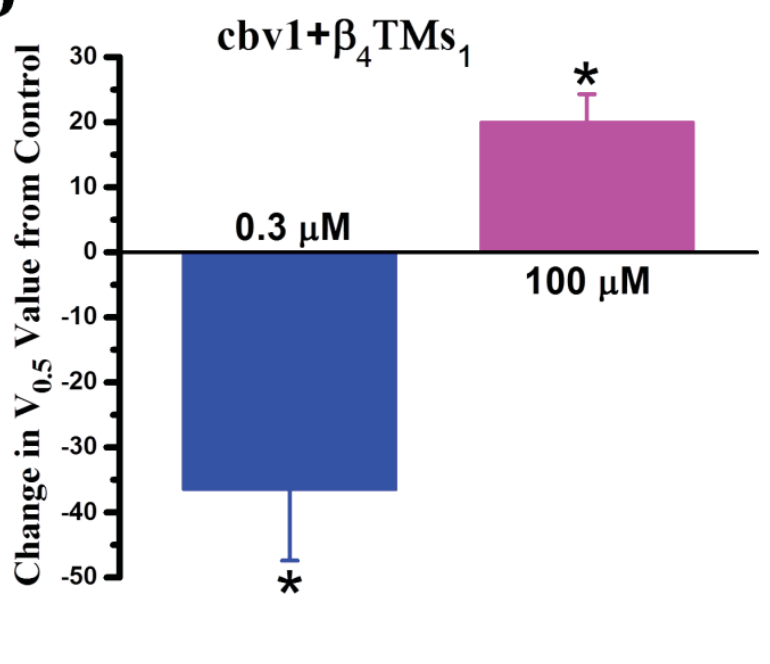

Figure 6-2. BK $\beta 1$ transmembrane domains determine ethanol-mediated inhibition of $\beta 1$-containing BK channel

Notes: A) TMs from $\beta 4$ subunit on BK $\beta 1$ background shift the activation-to-inhibition crossover of ethanol responses to $\approx 20 \mu \mathrm{M} \mathrm{Ca}^{2+}{ }^{2}$, mimicking cbv1+ $\beta 4$ and homomeric cbv1 channel responses. B) Bar graph representing ethanol-induced change in $V_{0.5}$ values from control and $\beta_{1} \mathrm{TMs}_{4}$ obtained at 0.3 and $100 \mu \mathrm{M} \mathrm{Ca}^{2+}{ }_{i}$, respectively. C) TMs from $\beta 1$ on a BK $\beta 4$ background shift the activation-to-inhibition crossover of ethanol responses to $3 \mu \mathrm{M}\left[\mathrm{Ca}^{2+}{ }_{i}\right]$, mimicking cbv $1+\beta 1$ responses. D) Bar graph representing ethanol-induced change in $\mathrm{V}_{0.5}$ values from control and $\beta_{4} \mathrm{TMs}_{1}$ obtained at 0.3 and 100 $\mu \mathrm{M} \mathrm{Ca}^{2+}$ i, respectively. *Different from control $(\mathrm{P}<0.05)$. Error bars show SEM; each point represents the average of $\geq 4$ patches. 


\subsubsection{Second Transmembrane Domain of BK- $\beta 1$ Subunit Determines the Ethanol- Induced Inhibition of BK Channels}

To identify whether a particular BK $\beta 1 \mathrm{TM}$ was sufficient to determine the ethanol action on $\beta 1$-containing BK channels, we first co-expressed with cbv1 a $\beta 1 / \beta 4$ chimera that contained TM1 from $\beta 1$ on $\beta 4$ background $\left(\beta_{4} \mathrm{TM} 1_{1}\right)$. Data demonstrate that ethanol increased the activity of these heterooligomeric channel at low $\left[\mathrm{Ca}^{2+}\right]$ i.e., $0.3-10$ $\mu \mathrm{M}$, which is evident as a significant decrease in $\mathrm{V}_{0.5}$ from the $\mathrm{V}_{0.5}-\left[\mathrm{Ca}^{2+}\right]$ plot. As $\left[\mathrm{Ca}^{2+}\right]$ increased, this activation turned into mild inhibition, evident from a small increase in $\mathrm{V}_{0.5}$. Furthermore, ethanol-induced activation-to-inhibition cross-over occurred at $\sim 20$ $\mu \mathrm{M}$ (Figure 6-3A and $\mathbf{B}$ ). Thus, the ethanol modulation cbv $1+\beta_{4} \mathrm{TM} 1_{1}$ channel activity follows a pattern that matches that of homomeric BK channels. These results seem to indicate that BK $\beta 1$ TM1 is not critical for ethanol-induced inhibition of $\beta 1$-containing BK channels. Collectively, this finding and the results obtained with $\beta_{4} \mathrm{TMs}_{1}$ raise the hypothesis that $\beta 1$ TM2 plays an important role in $\beta 1$ subunit modulation of ethanol action.

Thus, we co-expressed with cbv1 a $\beta 1 / \beta 4$ chimera that contained TM2 from $\beta 1$ on $\beta 4$ background $\left(\beta_{4} \mathrm{TM} 2_{1}\right)$ and studied the ethanol response of the resulting channels. Data show that ethanol increases the activity of these channel complexes at low $\left[\mathrm{Ca}^{2+}\right]$ i.e., $0.3 \mu \mathrm{M}$. As $\left[\mathrm{Ca}^{2+}\right]$ increased, this activation turned to significant inhibition, resulting in an activation-to-inhibition cross over at $\sim 3 \mu \mathrm{M}$ (Figure 6-3C and $\mathbf{D}$ ). This crossover matched that of cbv $1+\beta 1$ channels, suggesting that $\beta 1$ TM2 is critical for $\beta 1$ subunits to facilitate ethanol-mediated inhibition of BK channels.

After identifying via patch-clamp electrophysiology studies the specific region of $\beta 1$ subunit that was responsible for facilitating ethanol-induced inhibition of BK channels, we decided to determine any possible contribution e impact of such region to BK channel-mediated ethanol action on organ function, i.e., the cerebral artery. Thus, I next tested the hypothesis that "ethanol-induced constriction of cerebral arteries is critically dependent on specific region(s) of the arterial smooth muscle BK $\beta 1$-subunit". This aim includes studies on organ function under physiological conditions with the combination of reversible permeabilization and myogenic tone experiments. To our knowledge, this experimental approach was the first to address the role of a specific domain of $\beta 1$ subunit in ethanol action on organ function in alcohol research.

\subsubsection{Reversible Permeabilization Leads to Overexpression of Desired Proteins and Resulting Functional Middle Cerebral Arteries}

In order to achieve this goal, I took advantage of the reversible permeabilization technique (Lesh et al. 1995; Xi et al. 2008; Adebiyi et al. 2011; Bannister et al. 2011; Thomas-Gatewood et al. 2011). Using this technique, expressing vectors containing $\beta 1$ or $\beta 1 / \beta 4$ chimeras (Figure 6-1) were transfected into de-endothelized middle cerebral arteries of $K C N M B 1 \mathrm{~K} / \mathrm{O}$ mouse in order to express heteromeric $\mathrm{BK} \beta 1$ alone or $\beta 1 / \beta 4$ chimeras following protocols described elsewhere (see Chapter 3 ) . 
A

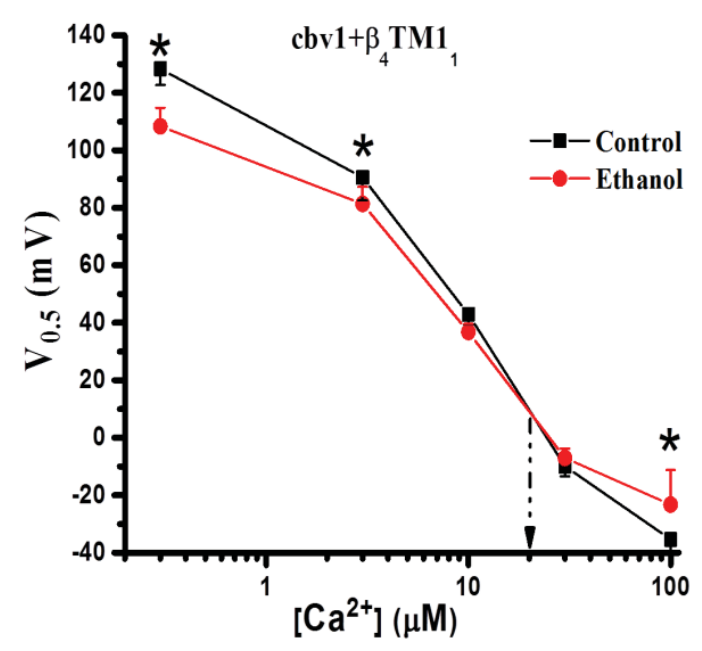

C

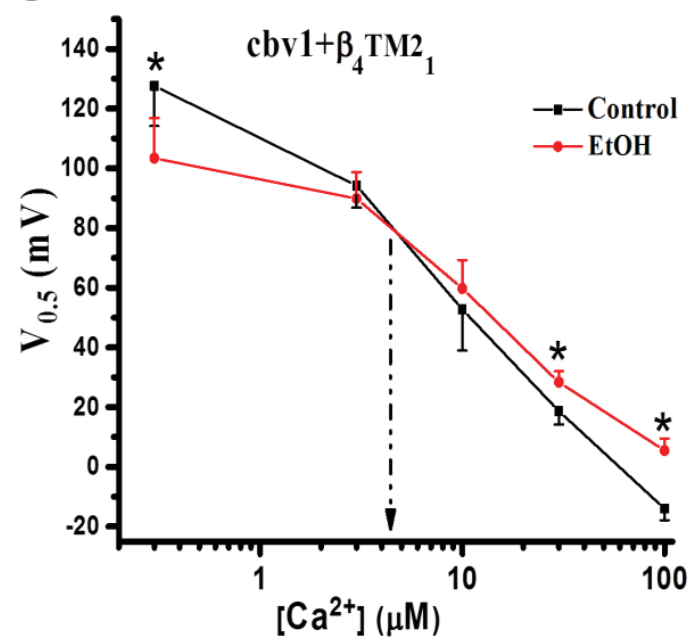

B

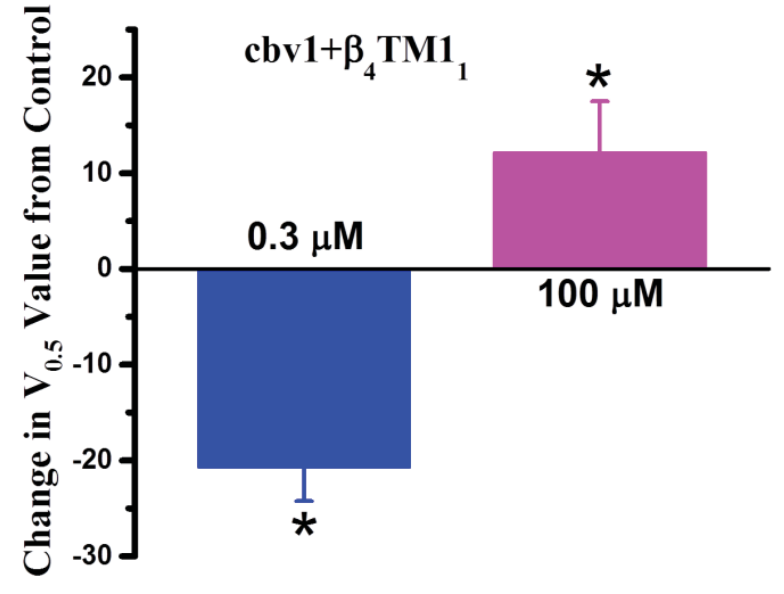

D

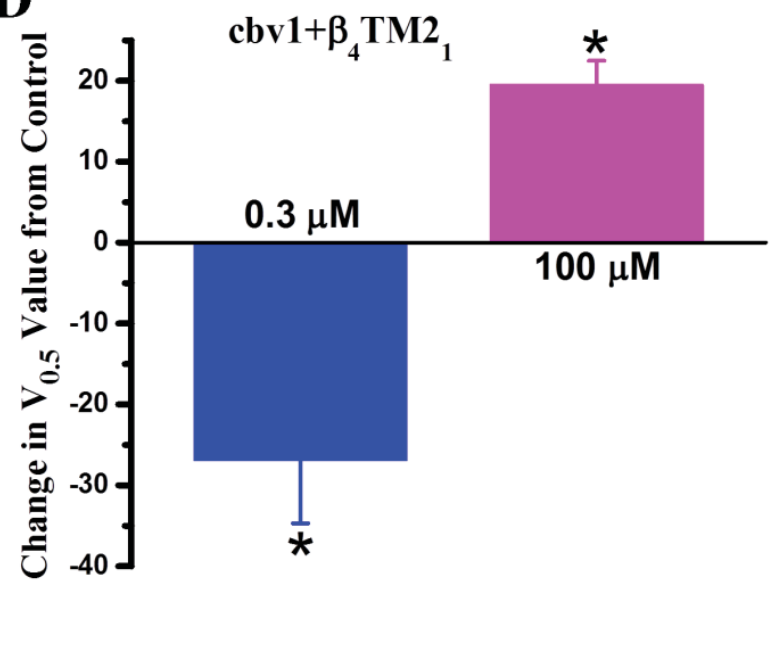

Figure 6-3. Second transmembrane domain of BK $\beta 1$ subunit determines the ethanol-induced inhibition of $\beta 1$-containing $B K$ channel

Notes: A) TM1 domain from $\beta 1$ on $\beta 4$ background shifts the activation-to-inhibition crossover of ethanol responses to $\approx 20 \mu \mathrm{M}\left[\mathrm{Ca}^{2+}{ }_{\mathrm{i}}\right]$, mimicking cbv $1+\beta 4$ or homomeric $\mathrm{BK}$ channel responses. B) Bar graph representing ethanol-induced change in $\mathrm{V}_{0.5}$ values from control and $\beta_{4} \mathrm{TM}_{1}$ obtained at 0.3 and $100 \mu \mathrm{M} \mathrm{Ca}^{2+}{ }^{2}$, respectively. C) TM2 from $\beta 1$ on $\beta 4$ background shifts the activation-to-inhibition crossover of ethanol responses to $3 \mu \mathrm{M}$ $\left[\mathrm{Ca}^{2+}{ }_{i}\right]$, mimicking cbv $1+\beta 1$ responses. D) Bar graph representing ethanol-induced change in $\mathrm{V}_{0.5}$ values from control and $\beta_{4} \mathrm{TM} 2_{1}$ at $0.3 \mu \mathrm{M}$ and $100 \mu \mathrm{M}$, respectively. *Different from control $(\mathrm{P}<0.05)$. Error bars show SEM; each point represents the average of $\geq 4$ patches. 
After 3-4 days of transfection, 1) successful overexpression of protein and 2) further resulting functional arteries were tested. First, successful reversible permeabilization resulting in protein overexpression was confirmed by western blotting. Arteries subjected to reverse permeabilization expressed significantly higher levels of BK $\beta 1$ alone (Figure 6-4A) or $\beta 1 / \beta 4$ chimeras (Figure 6-4B) than those of control arteries transfected with empty vectors. These results underscore the effectiveness of reversible permeabilization to overexpress selective proteins, as previously demonstrated for other proteins and tissues (Lesh et al. 1995; Xi et al. 2008; Bannister et al. 2011; ThomasGatewood et al. 2011).

Next, we determined whether reversible permeabilization resulted in loss of arterial function. After 4 days of reversible permeabilization with either $w t \beta 1$ or $\beta 1 / \beta 4$ chimeras using the protocols proposed in SA4 (see Chapter 3), I evaluated the artery diameter response to $60 \mathrm{mM} \mathrm{K}^{+}$and vasodilation in response to $\mathrm{Ca}^{2+}$-free bath solution to determine the vessel contractile response to depolarization and passive diameter, respectively. Figure 6-4C demonstrates one representative trace of cerebral artery diameter following organ transfection with $w t \beta 1$. A summary of $\mathrm{KCl}$-induced constrictive responses of middle cerebral arteries transfected with either $\beta 1$ alone or $\beta 1 / \beta 4$ chimeras is shown in Figure 6-4D. Collectively, these results suggest that reversible permeabilization technique in our hands results in overexpression of BK $\beta 1$ or $\beta 1 / \beta 4$ chimeras and renders functional $K C N M B 1 \mathrm{~K} / \mathrm{O}$ arteries that constrict and dilate as untransfected arteries from $w t$ mouse do (Bukiya et al. 2009).

\subsubsection{Ethanol-Induced Cerebral Artery Constriction Is Determined by $\beta 1$ Subunit of BK Channels}

Once we established that: 1) $\beta 1$ subunit TM2 is largely responsible for facilitating ethanol-induced inhibition of BK channels, and 2) reversible permeabilization of $K C N M B 1 \mathrm{~K} / \mathrm{O}$ mouse middle cerebral arteries leads to overexpression of a $\beta$ protein construct without perturbing vasomotion, we set to determine the contribution of BK $\beta 1$ subunit TM2 to this subunit facilitation of ethanol-induced cerebral constriction.

We addressed this question using myogenic tone experiments identical to the protocols mentioned in Chapter 3. Briefly, after 4 days of permeabilization, a $2 \mathrm{~mm}$ long artery segment will be pressurized to $60 \mathrm{mmHg}$ to develop myogenic tone and then vessel external diameter was continuously measured in response to application of following drugs: 1) depolarizing agent (60 mM KCl), 2) $50 \mathrm{mM}$ ethanol, 3) $45 \mu \mathrm{M} \mathrm{LCA}$, and 4) $\mathrm{Ca}^{2+}$ - free solution with sufficient wash in between each drug application (Figure 6-5).

First, we probed the arteries from wild type mice (C57BL/6 mice) with $50 \mathrm{mM}$ ethanol, which caused vasoconstriction of $7.96 \pm 0.82 \%$ from pre-ethanol application level (Figure 6-5A and $\mathbf{C}$ ). This response was used as positive control. These results are in agreement with previously published results (Bukiya et al. 2009; Bukiya et al. 2011). 

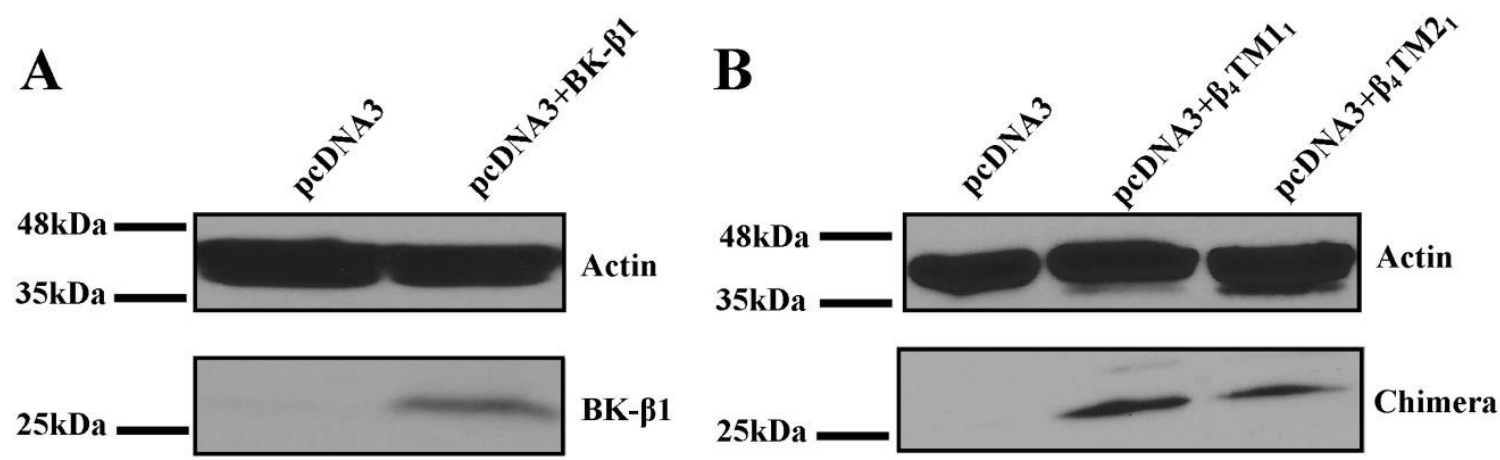

C

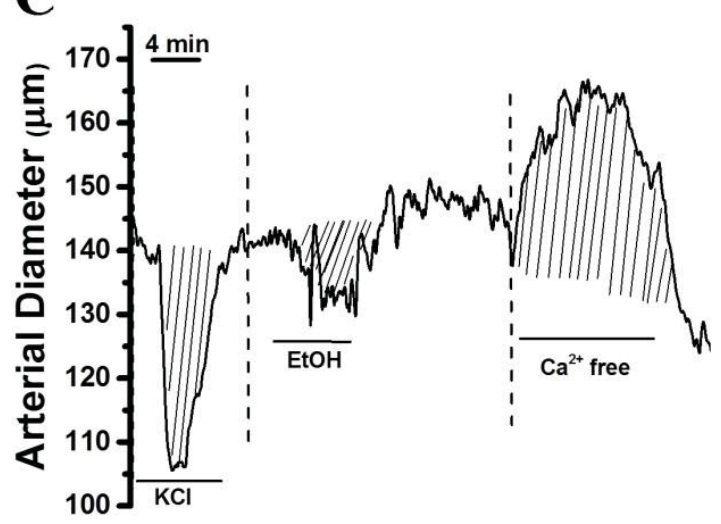

D KCl-Induced Middle Cerebral Artery Constriction

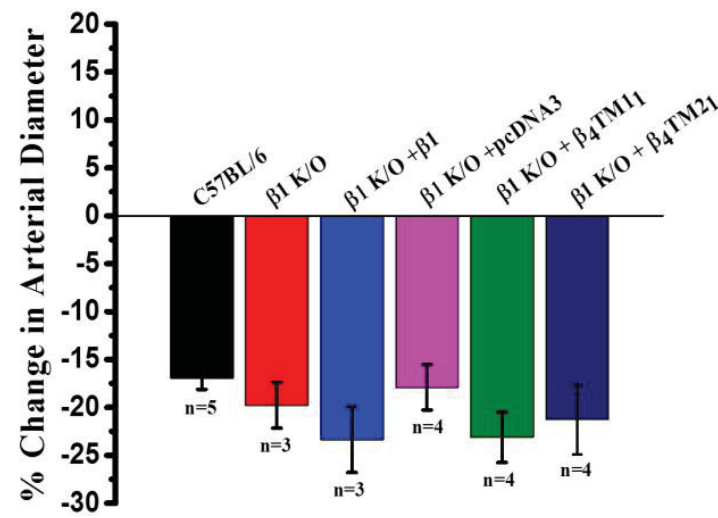

Figure 6-4. Reversible permeabilization leads to overexpression BK $\beta 1$ or $\beta 1 / \beta 4$ chimeric protein and functioning mouse middle cerebral artery

Notes: A) Representative western blots showing overexpression of BK- $\beta 1$ proteins in arteries transfected with empty vector (left lane) or pcDNA3+BK $\beta 1 \mathrm{cDNA}$ (right lane). B) Representative western blots showing overexpression of $\beta 1 / \beta 4$ chimeric proteins in arteries transfected with empty vector (left lane) or pcDNA3 $+\beta_{4} \mathrm{TM} 1_{1}$ (middle lane) or pcDNA3 $+\beta_{4}$ TM2 ${ }_{1}$ chimera cDNA.. C) Diameter trace from de-endothelized, pressurized middle cerebral artery after transfected with BK $\beta 1$. After development of myogenic tone, application of $60 \mathrm{mM} \mathrm{KCl}$ causes reversible constriction; $50 \mathrm{mM}$ ethanol produces constriction and $\mathrm{Ca}^{2+}$-free solution causes dilation. All these responses are similar to those obtained in arteries from wt mouse (Bukiya et al. 2009); D) Bar graph showing averaged decrease in cerebral arterial diameter (in \% change from pre- $\mathrm{KCl}$ diameter value) in response to $60 \mathrm{mM} \mathrm{KCl}$ after transfecting with various constructs as indicated; $w t$ arteries from C57BL/6 mouse. 
A

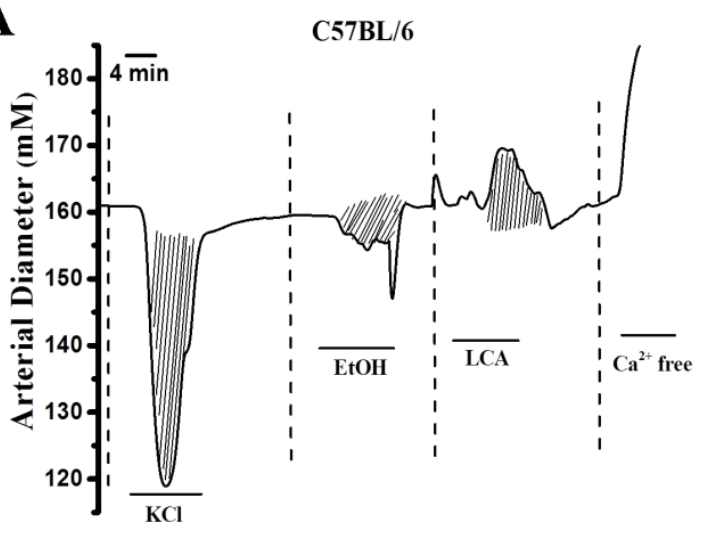

C

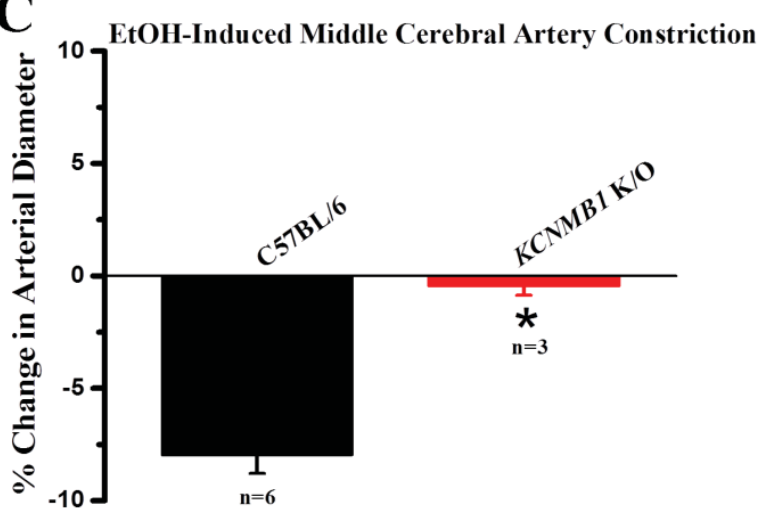

B

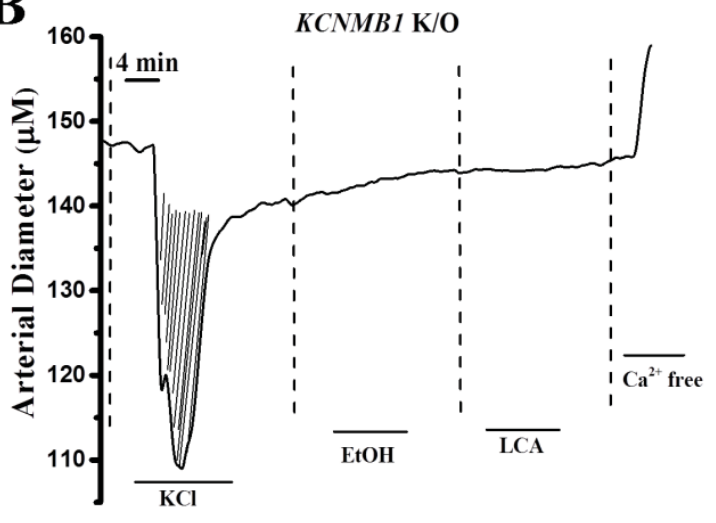

D

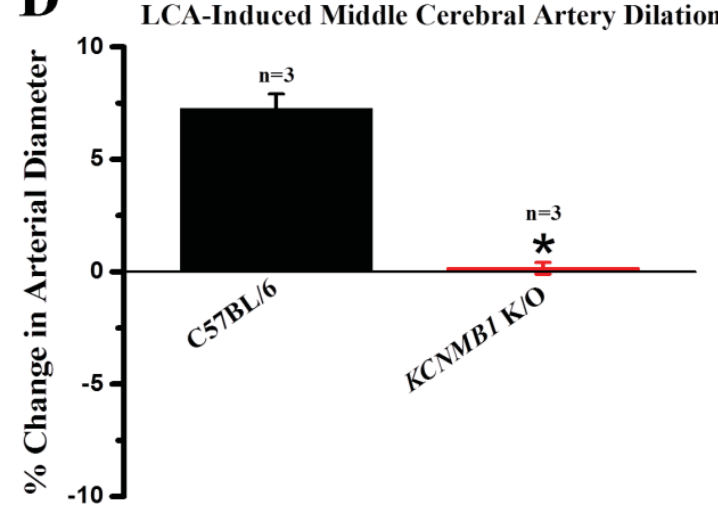

Figure 6-5. BK $\beta 1$ subunit determines ethanol-induced constriction of middle cerebral artery

Notes: A) Diameter trace from de-endothelized, pressurized middle cerebral artery from control (C57BL/6) mouse. After development of myogenic tone, application of $50 \mathrm{mM}$ ethanol causes reversible constriction; $45 \mu \mathrm{M}$ LCA produces dilation. B) However, arteries from $K C N M B 1 \mathrm{~K} / \mathrm{O}$ mouse failed to respond to acute application of $50 \mathrm{mM}$ ethanol or $45 \mu \mathrm{M}$ LCA. C, D) Bar graph showing percentage change in diameter in response to application of $50 \mathrm{mM}$ ethanol (C) and $45 \mu \mathrm{M} \mathrm{LCA}(\mathrm{D})$ on arteries obtained control and KCNMB1 K/O mice. *Different from control $(\mathrm{P}<0.05)$. Error bars show SEM; each point represents the average of 4-7 arteries. 
Moreover, this ethanol action is similar to the constriction of middle cerebral artery in rats constriction (Liu et al. 2004), buttressing the fact that the primary mechanisms mediating ethanol action on cerebral arteries are conserved among the two species. After ethanol application, $w t$ mouse arteries were probed with $45 \mu \mathrm{M}$ LCA, which caused $7.23 \pm 0.68 \%$ dilation (Figure 6-5A and $\mathbf{D}$ ). Again, these results are also consistent with previously reported studies (Bukiya et al. 2013).

Next, we tested the ethanol and LCA action on arteries obtained from KCNMB1 $\mathrm{K} / \mathrm{O}$ mice and $K C N M B 1 \mathrm{~K} / \mathrm{O}$ mice arteries transfected with empty vector, which were considered as our negative control. Indeed, and in contrast to $w t$ mouse arteries, ethanol consistently failed to constrict cerebral arteries from $K C N M B 1 \mathrm{~K} / \mathrm{O}$ mouse and arteries transfected with empty vector (Figure 6-5B and C, Figure 6-6A and Figure 6-7A). These results indicate that BK $\beta 1$ subunits are the primary determinants of alcoholinduced, cerebrovascular constriction. Furthermore, in contrary to arteries from $w t$ mouse, LCA also failed to cause dilation of cerebral arteries obtained from $K C N M B 1$ $\mathrm{K} / \mathrm{O}$ mouse (Figure 6-5B and D, Figure 6-6A and Figure 6-7B). Because LCA evokes BK channel activation via $\beta 1$ but not other beta subunits ( $\beta 2-4)$ (Bukiya et al. 2009). Further, it was already shown that amino acid residues in TM2 of BK $\beta 1$ subunit is interacts with LCA to bring about cerebral artery dilation (Bukiya et al. 2011). Therefore, we used LCA as a tool to identify the presence of BK $\beta 1$ subunit or TM2 region from $\beta 1$ subunit. Our results indicate that BK $\beta 1$ is responsible for LCA-induced cerebrovascular dilation via activation of smooth muscle cell BK channels. All These results are in agreement with previously published reports (Bukiya et al. 2008; Bukiya et al. 2009; Bukiya et al. 2013).

\subsubsection{BK $\beta 1$ Subunit Is Second Transmembrane Domain Is Necessary for Ethanol- Induced Cerebral Artery Constriction}

First, we transfected arteries from $K C N M B 1 \mathrm{~K} / \mathrm{O}$ mouse with $\mathrm{BK} \beta 1$ subunit and measured changes in arterial diameter in response to $50 \mathrm{mM}$ ethanol and $45 \mu \mathrm{M}$ LCA to test whether BK $\beta 1$ subunit transfected arteries were able to recover sensitivity to ethanol and LCA. Indeed, data demonstrated that ethanol-induced arterial constriction in KCNMB $1 \mathrm{~K} / \mathrm{O}$ mouse arteries transfected with BK $\beta 1$ (Figure 6-6B and Figure 6-7A) reached $7.76+1.2 \%$, which is similar to that evoked in control $(\mathrm{C} 57 \mathrm{BL} / 6)$ mouse. Likewise, LCA-induced artery dilation reached $8.41+2.6 \%$

(Figure 6-6B and Figure 6-7B), which is similar to that observed in control (C57BL/6) mice arteries (Figure 6-5A and $\mathbf{C}$ ). These results confirm the finding that $\mathrm{BK} \beta 1$ subunit is necessary for ethanol-induced constriction and LCA-induced dilation of cerebral artery.

After finding that TMs regions are important in controlling BK channel gating (Kuntamallappanavar et al., 2014) and that beta1 TM2 but not TM1 is largely responsible for $\beta 1$-induced facilitation of ethanol inhibition of BK channels (see section 6.2.3), we addressed the roleTM1 and TM2 of BK $\beta 1$ subunit in ethanol-induced constriction of cerebral arteries. 


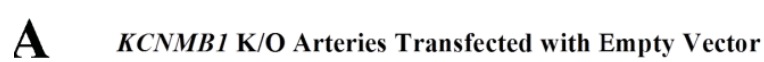

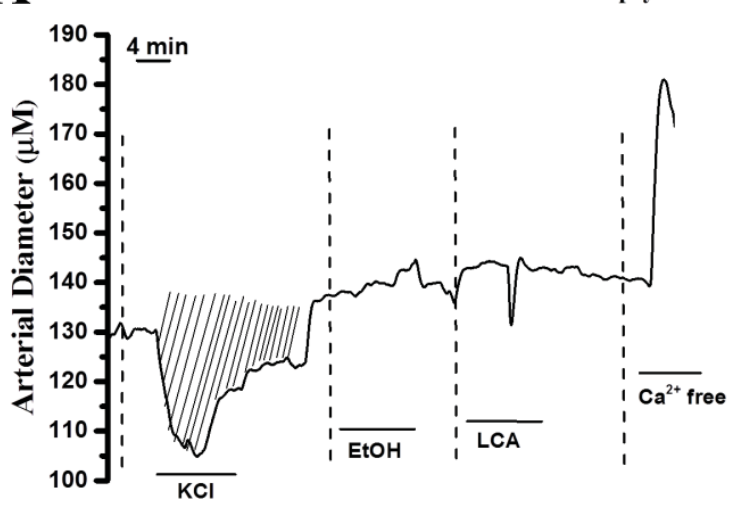

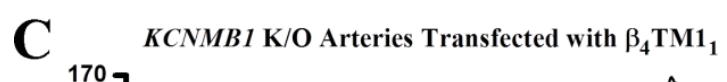

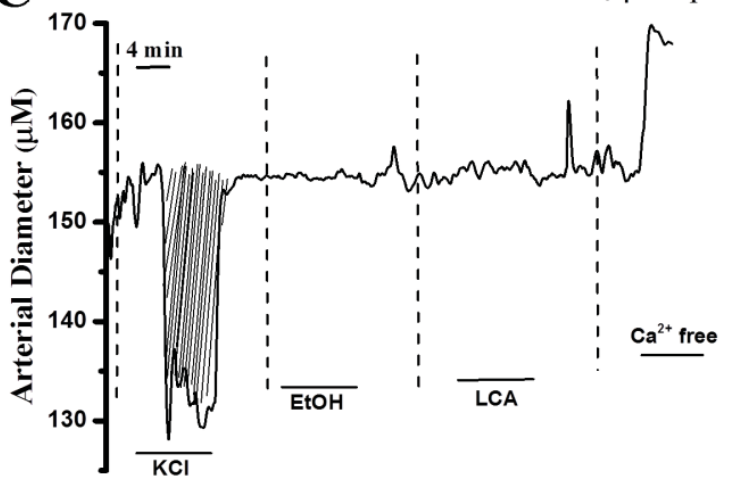

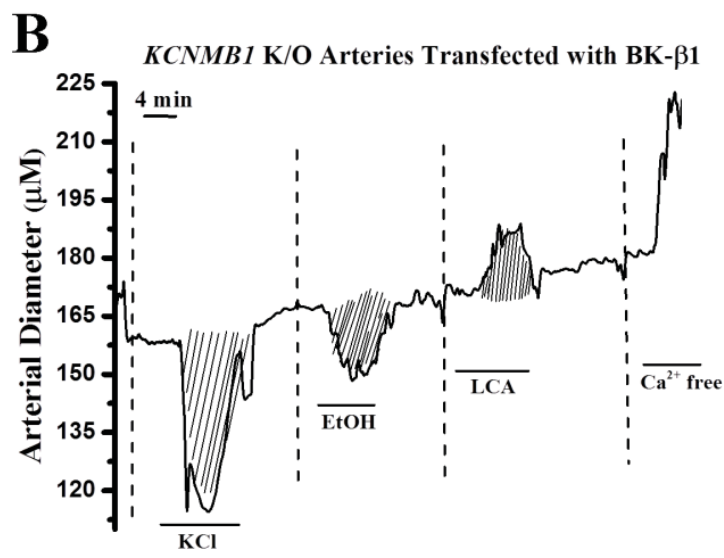

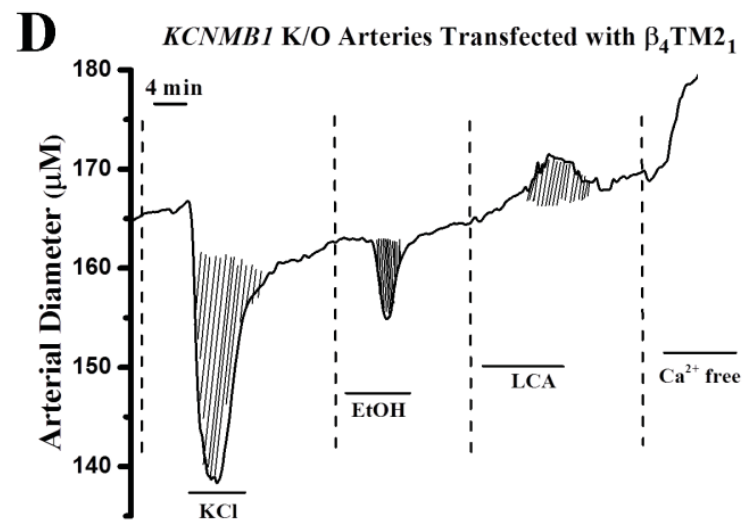

Figure 6-6. Second transmembrane domain of BK $\beta 1$ subunit determines ethanolinduced constriction of middle cerebral artery

Notes: A) $K C N M B 1 \mathrm{~K} / \mathrm{O}$ arteries transfected with empty vector failed to respond to application of $50 \mathrm{mM}$ ethanol and $45 \mu \mathrm{M}$ LCA. B) Diameter trace from de-endothelized, pressurized $K C N M B 1 \mathrm{~K} / \mathrm{O}$ middle cerebral artery transfected with BK $\beta 1$. Application of $50 \mathrm{mM}$ ethanol caused reversible constriction; $45 \mu \mathrm{M}$ LCA evokes artery dilation, which mimics the responses from control (C57BL/6) mice arteries. C) However, KCNMB1 K/O arteries transfected with $\beta_{4} \mathrm{TM} 1_{1}$ failed to respond to application of $50 \mathrm{mM}$ ethanol and $45 \mu \mathrm{M}$ LCA. C) Interestingly, $K C N M B 1 \mathrm{~K} / \mathrm{O}$ arteries transfected with $\beta_{4} \mathrm{TM} 2_{1}$ responded with constriction and dilation to application of $50 \mathrm{mM}$ ethanol and $45 \mu \mathrm{M} \mathrm{LCA}$, respectively, these responses matching those from control (C57BL/6) mice arteries. 


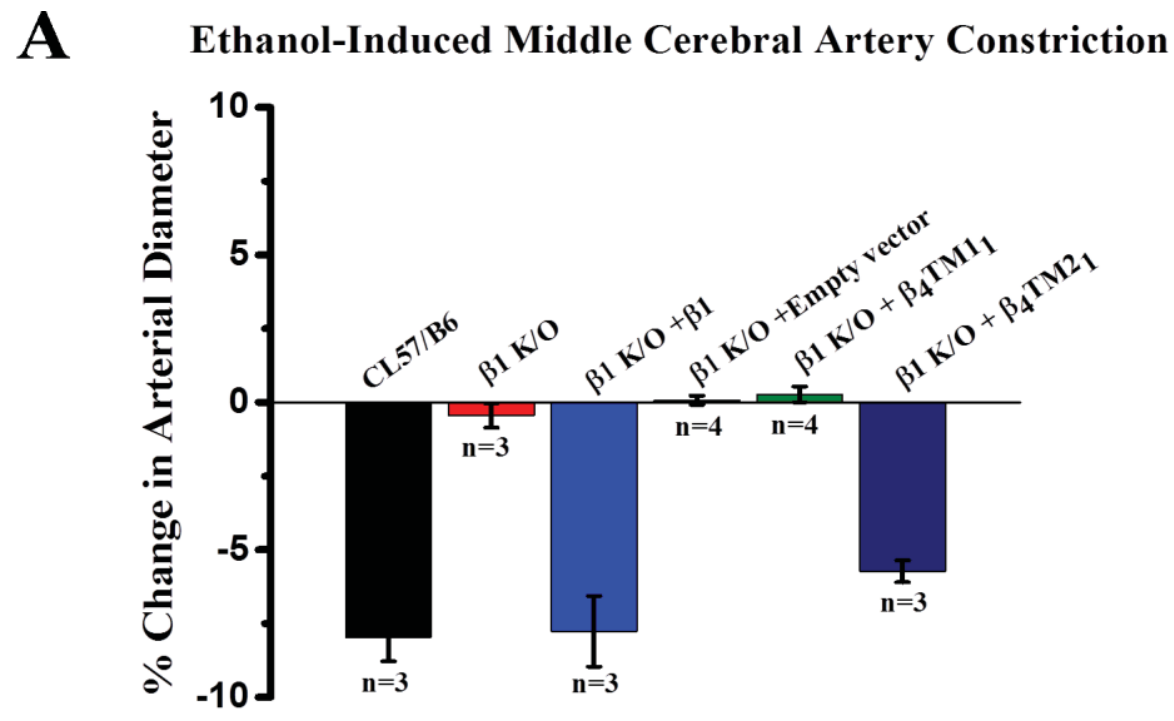

B Lithocholate-Induced Middle Cerebral Artery Dilation

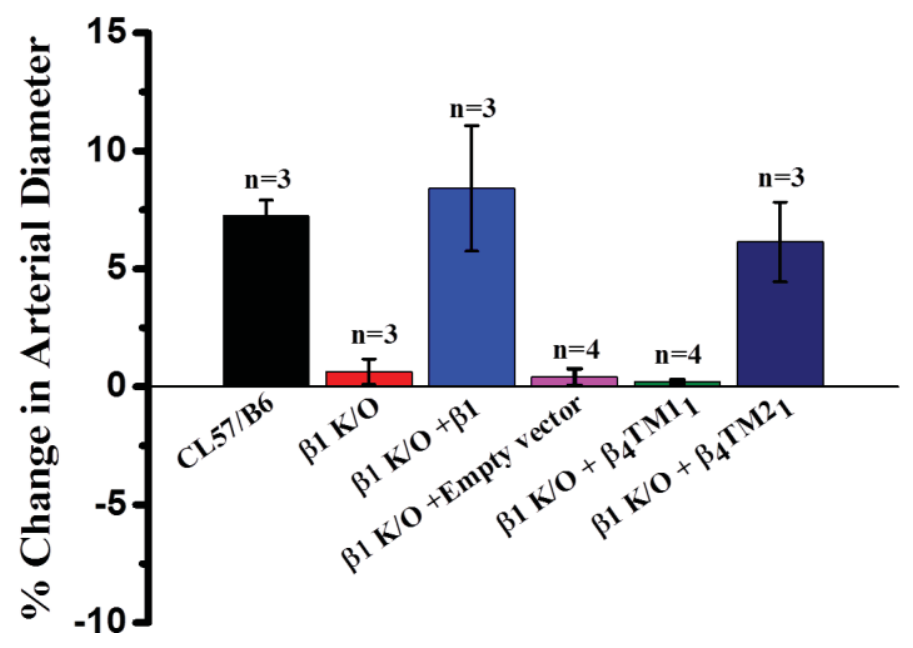

Figure 6-7. Second transmembrane domain of BK $\beta 1$ subunit is responsible for ethanol-induced constriction of middle cerebral artery

Notes: A, B) Bar graph showing percentage change in diameter in response to application of $50 \mathrm{mM}$ ethanol (A) and $45 \mu \mathrm{M}$ LCA (B) from $K C N M B 1 \mathrm{~K} / \mathrm{O}$ mice arteries transfected with different constructs as indicated. *Different from control (CL57B/6) $(\mathrm{P}<0.05)$. Error bars show SEM; each point represents the average of 4-7 arteries. 
Thus, we transfected KCNMB1 K/O mouse arteries with $\beta 4 \mathrm{TM} 11$ chimeric construct and then measured changes in arterial diameter in response to application of ethanol and LCA (Figure 6-6C and D).In contrast to the control (C57BL/6) mice arteries and arteries transfected with BK- $\beta 1$, ethanol consistently failed to constrict $K C N M B 1$ $\mathrm{K} / \mathrm{O}$ mouse arteries transfected with $\beta_{4} \mathrm{TM} 1_{1}$ (Figure 6-6C, and Figure 6-7A). These results indicate that EC loop, TM1 region and IC ends of BK $\beta 1$ subunit do not mediate ethanol-induced cerebral artery constriction. Application of LCA also failed to evoke dilation of these arteries (Figure 6-6C and Figure 6-7B). This is an expected result, as LCA action on BK channels requires $\beta 1$ TM2 (Bukiya et al. 2011), which is absent in the $\beta_{4} \mathrm{TM} 1_{1}$ chimera.

Finally, we determined the contribution of BK $\beta 1$ subunit TM2 to ethanolinduced constriction of cerebral arteries. Thus, we transfected $K C N M B 1 \mathrm{~K} / \mathrm{O}$ mouse cerebral arteries with the $\beta_{4} \mathrm{TM} 2_{1}$ chimera and measured changes in arterial diameter in response to application of $50 \mathrm{mM}$ ethanol and $45 \mu \mathrm{M}$ LCA. In contrast to the arteries from $K C N M B 1$ knock-out mice, ethanol constricted cerebral arteries transfected with $\beta_{4} \mathrm{TM} 2_{1}$ by $5.73+0.37 \%$ (Figure 6-6D and Figure 6-7A). These results indicate that BK $\beta 1$ subunit TM2 is critical for the ethanol-induced cerebral artery constriction. Further, functional expression of $\beta_{4} \mathrm{TM} 2_{1}$ and its coupling to the BK channel-forming subunit chimera was confirmed by the artery diameter response to LCA application. The latter caused dilated these arteries by $6.14+1.69 \%$ (Figure 6-6D and Figure 6-7B). This response is almost identical to the response to LC from control $(\mathrm{C} 57 \mathrm{BL} / 6)$ and $K C N M B 1$ $\mathrm{K} / \mathrm{O}$ mouse arteries transfected with $\beta 1$. These results seem to indicate that of BK $\beta 1$ subunit TM2 region is necessary for ethanol-induced cerebral artery constriction.

\subsection{Discussion}

Using a combination of patch-clamp electrophysiology on BK channels made of cbv1 and $w t \beta 1$ or chimeric $\beta 1 / \beta 4$ subunits, reversible permeabilizaton and in vitro pressurization of rat cerebral arteries, we identified for the first time the specific domain of the BK channel regulatory $\beta 1$ subunit that is critical for ethanol-induced inhibition of channel activity and artery constriction mediated by this channel type. Indeed, our results clearly demonstrate that $\beta 1$ TM2 is critical for ethanol-mediated action on BK channels and cerebral artery constriction. To our knowledge, this is the first time that a specific domain of an accessory subunit of any member of the voltage-activated TM6 ion channel super family is shown critical for ethanol action on ion channel function and vascular physiology.

Two studies have mapped alcohol-sensing sites to intracellular domains of the channel-forming subunit, as reported for GIRK (Aryal et al. 2009) and recently for BK themselves by us (Bukiya et al. 2014). These findings are rather unique, as multiple groups have previously documented that it is up to transmembrane regions to provide alcohol-sensing sequences in ion channels, as proposed for GABA-A, glycine, nACh and prokaryotic Gleobacter violaceus (GLIC) ionotropic receptor. We are hereby documenting for the first time that ethanol functionally targets a TM region of an 
auxiliary subunit of BK channel to modulate channel activity and eventually rendering cerebral artery constriction.

As we proposed in previous chapter, at least three structural interpretations on this crucial role of a $\beta 1$ subunit domain (TM2) on ethanol action ("anti- $\beta 1$ ") are possible: 1 ) $\beta 1$ subunit TM2 itself possess an ethanol recognition/interaction site(s) and thereby perturbs the functional coupling between slo1 (cbv1 in our case) and $\beta 1$ subunits upon ethanol binding to this site; 2) newly identified ethanol sensing site K361 (slo1 "activation site") (Bukiya et al. 2014) in the cytosolic tail of the slol channel may serve as an "inhibition site", ethanol interaction with this site may disrupt the functional coupling between slo1 and $\beta 1$ subunit; 3) Ethanol disrupts the lipid around the channel complex, TM2 in particular, and thereby perturbs the functional coupling between slo 1 and $\beta 1$ subunits, which involves TM2 (Kuntamallappanavar et al. 2014). Disregarding the structural specifics, it is clear from out data that $\beta 1$ subunit TM2 plays a critical role in ethanol action on $\beta 1$-containing BK channels and eventual cerebral artery constriction.

From a structural point of view, present data may provide further insights on ethanol modulation of specific BK- $\beta 1$ protein domains that participate in basal slo1- $\beta 1$ coupling. Chimeric channel studies between mslo and dslo have demonstrated that slo1 $\mathrm{N}$-end and S0 are both critical for the functional effects of $\beta 1$ (Wallner et al. 1996; Morrow et al. 2006; Lee et al. 2010). Disulfide cross-linking studies shown that Nterminal EC end of S0 is in close proximity of S3 and S4 during resting state and move in concert during voltage sensor activation (Wu et al. 2009; Liu et al. 2010; Wu et al. 2013). Amino acid substitutions in S0 disrupt the voltage-dependent channel activation of BK channels, underscoring S0 interactions with other membrane-spanning domains of the channel VSD (Wallner et al. 1996). Similar disulfide cross-linking studies demonstrated that BK $\beta$ TM1 and TM2 are both packed close to each other at the mouth of the cleft between the VSDs of two adjacent slo1 subunits (Wu et al. 2009; Lee et al. 2010; Wu et al. 2013). Within this cleft, TM1 is close to S1 of one VSD and TM2 close to S0 of the adjacent VSD (Wu et al. 2009; Lee et al. 2010; Wu et al. 2013). The proposed location of both TM1 and TM2 and my own findings (Kuntamallappanavar et al., 2014) suggest that both TMs of $\beta 1$ subunit and slo1 VSD TMs are functionally coupled. Results of present study show that ethanol targets TM2 of $\beta 1$ subunit to disrupt the functional coupling between slo1 and $\beta 1$ subunit, thereby, antagonizing the functional effects of $\beta 1$ subunit. This speculation seems to be consistent with the results of HA model fitting that in presence of $\beta 1$ subunit, ethanol perturbs the allosteric interaction that involves RCKs and VSD (decrease in allosteric factor E). Thus decreasing channel open probability.

Current information will lead to consider TM2-targeting agents, whether endogenous steroids such as lithocholate (Bukiya et al. 2007; Bukiya et al. 2008; Bukiya et al. 2009; Bukiya et al. 2011) or synthetic agents such as hydroxyl-alkynoic acids and HENA (Bukiya et al. 2013) that target $\beta 1$ TM2 and thus, oppose alcohol-induced cerebral artery constriction. Future studies using point mutagenesis and pharmacophore design will help to a better rationale design of novel agents that would counteract such ethanol action without altering organ physiology that is not dependent on BK $\beta 1$ subunits. 


\subsection{Overall Summary of Results}

1. Slo1sensitivity to ethanol channel is dependent on $\mathrm{Ca}^{2+}$ but not $\mathrm{Mg}^{2+}$, even when the latter is present at levels that do activate slolchannels.

2. Activating ion is required for and modulates ethanol final on slo1 channel activity while being unable to embold slo2 or slo3 channels with ethanol sensitivity.

3. Ethanol inhibition of BK channels is facilitated by $\beta 1, \beta 2$ and $\beta 2$-IR subunits.

4. The inactivation domain of $\beta 2$ does not play a major role in ethanol action.

5. BK $\beta 3$ and $\beta 4$ subunits fail to alter ethanol overall effect on - homomeric slo1 (cbv1) channels.

6. Ethanol-induced inhibition of $\beta 1$-containing $\mathrm{BK}$ channels is allosterically coupled to $\mathrm{Ca}^{2+}{ }_{\mathrm{i}}$-driven gating.

7. Ethanol-induced modification of activity of slo1 (cbv1) is due to modification of $\mathrm{Ca}^{2+}{ }_{\mathrm{i}}$ binding affinity wherease, ethanol-induced modification of heteromeric cbv $1+\beta 1$ BK channels is primarily due to alteration in allosteric interaction between 1) RCK and voltage sensing domains and 2) RCKs and pore gate domains.

8. $\beta 1 \mathrm{TM} 2$ is critical for ethanol to inhibit vascular smooth muscle cell BK channels and drug-induced cerebral artery constriction.

\subsection{Final Conclusion}

BK $\beta 1$ TM2 enables ethanol-induced inhibition of $\beta 1$-conaining BK channels and cerebral artery constriction, with drug action on channel activity being dependent on modification of calcium-gating parameters.

\subsection{Future Directions}

Our study shows that a specific region of BK- $\beta 1$ subunit critically controls ethanol-induced inhibition of BK channels and its involvement in cerebral artery constriction. The following future studies are considered:

1. Identify the specific gating processes that are targeted by ethanol leading to differential modification of BK currents contains different $\beta$ subunit composition $(\beta 2-\beta 4)$.

2. Pinpoint mutagenesis strategies to identify possible amino acid residues in TM2 region of $\beta 1$ subunit that could be involved in sensing or interacting with ethanol molecules.

3. Design a pharmacophore based on amino acid interaction and then discover or design pharmacological agents that antagonize the effect of ethanol on cerebral artery constriction via BK $\beta 1$ subunits. 


\section{LIST OF REFERENCES}

Adebiyi, A., Narayanan, D. and Jaggar, J. H. (2011). "Caveolin-1 assembles type 1 inositol 1,4,5-trisphosphate receptors and canonical transient receptor potential 3 channels into a functional signaling complex in arterial smooth muscle cells." J Biol Chem 286(6): 4341-4348.

Altura, B. M. and Altura, B. T. (1982). "Microvascular and vascular smooth muscle actions of ethanol, acetaldehyde, and acetate." Fed Proc 41(8): 2447-2451.

Altura, B. M. and Altura, B. T. (1984). "Alcohol, the cerebral circulation and strokes." Alcohol 1(4): 325-331.

Altura, B. M., Altura, B. T. and Gebrewold, A. (1983). "Alcohol-induced spasms of cerebral blood vessels: relation to cerebrovascular accidents and sudden death." Science 220(4594): 331-333.

Amberg, G. C. and Santana, L. F. (2003). "Downregulation of the BK channel beta1 subunit in genetic hypertension." Circ Res 93(10): 965-971.

Arkwright, P. D., Beilin, L. J., Vandongen, R., Rouse, I. A. and Lalor, C. (1982). "The pressor effect of moderate alcohol consumption in man: a search for mechanisms." Circulation 66(3): 515-519.

Aryal, P., Dvir, H., Choe, S. and Slesinger, P. A. (2009). "A discrete alcohol pocket involved in GIRK channel activation." Nat Neurosci 12(8): 988-995.

Asano, M., Masuzawa-Ito, K. and Matsuda, T. (1993). "Charybdotoxin-sensitive K+ channels regulate the myogenic tone in the resting state of arteries from spontaneously hypertensive rats." Br J Pharmacol 108(1): 214-222.

Bannister, J. P., Thomas-Gatewood, C. M., Neeb, Z. P., Adebiyi, A., Cheng, X. and Jaggar, J. H. (2011). "Ca(V)1.2 channel N-terminal splice variants modulate functional surface expression in resistance size artery smooth muscle cells." J Biol Chem 286(17): 15058-15066.

Bao, L. and Cox, D. H. (2005). "Gating and ionic currents reveal how the BKCa channel's Ca2+ sensitivity is enhanced by its beta1 subunit." J Gen Physiol 126(4): 393412.

Barnett, N. P., Monti, P. M., Spirito, A., Colby, S. M., Rohsenow, D. J., Ruffolo, L. and Woolard, R. (2003). "Alcohol use and related harm among older adolescents treated in an emergency department: the importance of alcohol status and college status." J Stud Alcohol 64(3): 342-349. 
Beaglehole, R. (1990). "International trends in coronary heart disease mortality, morbidity, and risk factors." Epidemiol Rev 12: 1-15.

Behrens, R., Nolting, A., Reimann, F., Schwarz, M., Waldschutz, R. and Pongs, O. (2000). "hKCNMB3 and hKCNMB4, cloning and characterization of two members of the large-conductance calcium-activated potassium channel beta subunit family." FEBS Lett 474(1): 99-106.

Bhattacharjee, A. and Kaczmarek, L. K. (2005). "For K+ channels, Na+ is the new Ca2+." Trends Neurosci 28(8): 422-428.

Brayden, J. E. and Nelson, M. T. (1992). "Regulation of arterial tone by activation of calcium-dependent potassium channels." Science 256(5056): 532-535.

Brenner, R., Jegla, T. J., Wickenden, A., Liu, Y. and Aldrich, R. W. (2000). "Cloning and functional characterization of novel large conductance calcium-activated potassium channel beta subunits, hKCNMB3 and hKCNMB4." J Biol Chem 275(9): 6453-6461.

Brenner, R., Perez, G. J., Bonev, A. D., Eckman, D. M., Kosek, J. C., Wiler, S. W., Patterson, A. J., Nelson, M. T. and Aldrich, R. W. (2000). "Vasoregulation by the beta1 subunit of the calcium-activated potassium channel." Nature 407(6806): 870-876.

Brodie, M. S., Scholz, A., Weiger, T. M. and Dopico, A. M. (2007). "Ethanol interactions with calcium-dependent potassium channels." Alcohol Clin Exp Res 31(10): 1625-1632.

Bukiya, A. N., Kuntamallappanavar, G., Edwards, J., Singh, A. K., Shivakumar, B. and Dopico, A. M. (2014). "An alcohol-sensing site in the calcium- and voltage-gated, large conductance potassium (BK) channel." Proc Natl Acad Sci U S A 111(25): 9313-9318.

Bukiya, A. N., Liu, J. and Dopico, A. M. (2009). "The BK channel accessory beta1 subunit determines alcohol-induced cerebrovascular constriction." FEBS Lett 583(17): 2779-2784.

Bukiya, A. N., Liu, J., Toro, L. and Dopico, A. M. (2007). "Beta1 (KCNMB1) subunits mediate lithocholate activation of large-conductance $\mathrm{Ca} 2+$-activated $\mathrm{K}+$ channels and dilation in small, resistance-size arteries." Mol Pharmacol 72(2): 359-369.

Bukiya, A. N., McMillan, J. E., Fedinec, A. L., Patil, S. A., Miller, D. D., Leffler, C. W., Parrill, A. L. and Dopico, A. M. (2013). "Cerebrovascular dilation via selective targeting of the cholane steroid-recognition site in the BK channel betal-subunit by a novel nonsteroidal agent." Mol Pharmacol 83(5): 1030-1044.

Bukiya, A. N., Singh, A. K., Parrill, A. L. and Dopico, A. M. (2011). "The steroid interaction site in transmembrane domain 2 of the large conductance, voltage- and calcium-gated potassium (BK) channel accessory beta1 subunit." Proc Natl Acad Sci US A 108(50): 20207-20212. 
Bukiya, A. N., Vaithianathan, T., Kuntamallappanavar, G., Asuncion-Chin, M. and Dopico, A. M. (2011). "Smooth muscle cholesterol enables BK beta1 subunit-mediated channel inhibition and subsequent vasoconstriction evoked by alcohol." Arterioscler Thromb Vasc Biol 31(11): 2410-2423.

Bukiya, A. N., Vaithianathan, T., Toro, L. and Dopico, A. M. (2008). "The second transmembrane domain of the large conductance, voltage- and calcium-gated potassium channel beta(1) subunit is a lithocholate sensor." FEBS Lett 582(5): 673-678.

Bukiya, A. N., Vaithianathan, T., Toro, L. and Dopico, A. M. (2009). "Channel beta2-4 subunits fail to substitute for betal in sensitizing BK channels to lithocholate." Biochem Biophys Res Commun 390(3): 995-1000.

Callahan, A. (2001). "Cerebrovascular disease and statins: a potential addition to the therapeutic armamentarium for stroke prevention." Am J Cardiol 88(7b): 33j-37j.

Chen, H., Kronengold, J., Yan, Y., Gazula, V. R., Brown, M. R., Ma, L., Ferreira, G., Yang, Y., Bhattacharjee, A., Sigworth, F. J., Salkoff, L. and Kaczmarek, L. K. (2009). "The N-terminal domain of Slack determines the formation and trafficking of Slick/Slack heteromeric sodium-activated potassium channels." J Neurosci 29(17): 5654-5665.

Cheng, H. and Lederer, W. J. (2008). "Calcium sparks." Physiol Rev 88(4): 1491-1545.

Contreras, G. F., Neely, A., Alvarez, O., Gonzalez, C. and Latorre, R. (2012).

"Modulation of BK channel voltage gating by different auxiliary beta subunits." Proc Natl Acad Sci U S A 109(46): 18991-18996.

Cox, D. H. and Aldrich, R. W. (2000). "Role of the beta1 subunit in large-conductance $\mathrm{Ca}(2+)$-activated $\mathrm{K}(+)$ channel gating energetics. Mechanisms of enhanced $\mathrm{Ca}(2+)$ sensitivity." J Gen Physiol 116(3): 411-432.

Cox, D. H., Cui, J. and Aldrich, R. W. (1997). "Allosteric gating of a large conductance Ca-activated K+ channel." J Gen Physiol 110(3): 257-281.

Cox, D. H., Cui, J. and Aldrich, R. W. (1997). "Separation of gating properties from permeation and block in mslo large conductance Ca-activated $\mathrm{K}+$ channels." $J$ Gen Physiol 109(5): 633-646.

Crowley, J. J., Treistman, S. N. and Dopico, A. M. (2003). "Cholesterol antagonizes ethanol potentiation of human brain $\mathrm{BKCa}$ channels reconstituted into phospholipid bilayers." Mol Pharmacol 64(2): 365-372.

Crowley, J. J., Treistman, S. N. and Dopico, A. M. (2005). "Distinct structural features of phospholipids differentially determine ethanol sensitivity and basal function of BK channels." Mol Pharmacol 68(1): 4-10. 
Dopico, A. M. (2003). "Ethanol sensitivity of BK(Ca) channels from arterial smooth muscle does not require the presence of the beta 1-subunit." Am J Physiol Cell Physiol 284(6): C1468-1480.

Dopico, A. M., Anantharam, V. and Treistman, S. N. (1998). "Ethanol increases the activity of $\mathrm{Ca}(++)$-dependent $\mathrm{K}+(\mathrm{mslo})$ channels: functional interaction with cytosolic Ca++." J Pharmacol Exp Ther 284(1): 258-268.

Dopico, A. M., Bukiya, A. N. and Singh, A. K. (2012). "Large conductance, calcium- and voltage-gated potassium (BK) channels: regulation by cholesterol." Pharmacol Ther 135(2): 133-150.

Dopico, A. M., Chu, B., Lemos, J. R. and Treistman, S. N. (1999). "Alcohol modulation of calcium-activated potassium channels." Neurochem Int 35(2): 103-106.

Dopico, A. M., Lemos, J. R. and Treistman, S. N. (1996). "Ethanol increases the activity of large conductance, $\mathrm{Ca}(2+)$-activated $\mathrm{K}+$ channels in isolated neurohypophysial terminals." Mol Pharmacol 49(1): 40-48.

Dryer, S. E. (1994). "Na(+)-activated K+ channels: a new family of large-conductance ion channels." Trends Neurosci 17(4): 155-160.

Erdos, B., Miller, A. W. and Busija, D. W. (2002). "Alterations in KATP and KCa channel function in cerebral arteries of insulin-resistant rats." Am J Physiol Heart Circ Physiol 283(6): H2472-2477.

Feinberg-Zadek, P. L., Martin, G. and Treistman, S. N. (2008). "BK channel subunit composition modulates molecular tolerance to ethanol." Alcohol Clin Exp Res 32(7): 1207-1216.

Gil-Nunez, A. C. and Villanueva, J. A. (2001). "Advantages of lipid-lowering therapy in cerebral ischemia: role of HMG-CoA reductase inhibitors." Cerebrovasc Dis 11 Suppl 1: $85-95$.

Gonzalez-Perez, V., Xia, X. M. and Lingle, C. J. (2014). "Functional regulation of BK potassium channels by gammal auxiliary subunits." Proc Natl Acad Sci U S A 111(13): 4868-4873.

Gordon, E. L., Nguyen, T. S., Ngai, A. C. and Winn, H. R. (1995). "Differential effects of alcohols on intracerebral arterioles. Ethanol alone causes vasoconstriction." J Cereb Blood Flow Metab 15(3): 532-538.

Haapaniemi, H., Hillbom, M. and Juvela, S. (1997). "Lifestyle-associated risk factors for acute brain infarction among persons of working age." Stroke 28(1): 26-30. 
Horrigan, F. T. (2012). "Perspectives on: conformational coupling in ion channels: conformational coupling in BK potassium channels." J Gen Physiol 140(6): 625-634.

Horrigan, F. T. and Aldrich, R. W. (1999). "Allosteric voltage gating of potassium channels II. Mslo channel gating charge movement in the absence of $\mathrm{Ca}(2+)$." J Gen Physiol 114(2): 305-336.

Horrigan, F. T. and Aldrich, R. W. (2002). "Coupling between voltage sensor activation, $\mathrm{Ca} 2+$ binding and channel opening in large conductance $(\mathrm{BK})$ potassium channels." $J$ Gen Physiol 120(3): 267-305.

Horrigan, F. T., Cui, J. and Aldrich, R. W. (1999). "Allosteric voltage gating of potassium channels I. Mslo ionic currents in the absence of $\mathrm{Ca}(2+) . "$ J Gen Physiol 114(2): 277-304.

Hu, L., Shi, J., Ma, Z., Krishnamoorthy, G., Sieling, F., Zhang, G., Horrigan, F. T. and Cui, J. (2003). "Participation of the S4 voltage sensor in the Mg2+-dependent activation of large conductance (BK) K+ channels." Proc Natl Acad Sci U S A 100(18): 1048810493.

Iwase, S., Matsukawa, T., Ishihara, S., Tanaka, A., Tanabe, K., Danbara, A., Matsuo, M., Sugiyama, Y. and Mano, T. (1995). "Effect of oral ethanol intake on muscle sympathetic nerve activity and cardiovascular functions in humans." J Auton Nerv Syst 54(3): 206214.

Jackson, R., Stewart, A., Beaglehole, R. and Scragg, R. (1985). "Alcohol consumption and blood pressure." Am J Epidemiol 122(6): 1037-1044.

Jaggar, J. H., Li, A., Parfenova, H., Liu, J., Umstot, E. S., Dopico, A. M. and Leffler, C. W. (2005). "Heme is a carbon monoxide receptor for large-conductance Ca2+-activated $\mathrm{K}+$ channels." Circ Res 97(8): 805-812.

Jaggar, J. H. and Nelson, M. T. (2000). "Differential regulation of $\mathrm{Ca}(2+)$ sparks and $\mathrm{Ca}(2+)$ waves by UTP in rat cerebral artery smooth muscle cells." Am J Physiol Cell Physiol 279(5): C1528-1539.

Jaggar, J. H., Porter, V. A., Lederer, W. J. and Nelson, M. T. (2000). "Calcium sparks in smooth muscle." Am J Physiol Cell Physiol 278(2): C235-256.

Jakab, M., Weiger, T. M. and Hermann, A. (1997). "Ethanol activates maxi Ca2+activated $\mathrm{K}+$ channels of clonal pituitary (GH3) cells." J Membr Biol 157(3): 237-245.

Jiang, Y., Lee, A., Chen, J., Cadene, M., Chait, B. T. and MacKinnon, R. (2002). "The open pore conformation of potassium channels." Nature 417(6888): 523-526. 
Klatsky, A. L., Friedman, G. D. and Armstrong, M. A. (1986). "The relationships between alcoholic beverage use and other traits to blood pressure: a new Kaiser Permanente study." Circulation 73(4): 628-636.

Kuntamallappanavar, G., Toro, L. and Dopico, A. M. (2014). "Both transmembrane domains of BK beta1 subunits are essential to confer the normal phenotype of beta1containing BK channels." PLoS One 9(10): e109306.

Latorre, R., Oberhauser, A., Labarca, P. and Alvarez, O. (1989). "Varieties of calciumactivated potassium channels." Annu Rev Physiol 51: 385-399.

Lee, U. S. and Cui, J. (2010). "BK channel activation: structural and functional insights." Trends Neurosci 33(9): 415-423.

Lee, U. S., Shi, J. and Cui, J. (2010). "Modulation of BK channel gating by the ss2 subunit involves both membrane-spanning and cytoplasmic domains of Slo1." J Neurosci 30(48): 16170-16179.

Lesh, R. E., Somlyo, A. P., Owens, G. K. and Somlyo, A. V. (1995). "Reversible permeabilization. A novel technique for the intracellular introduction of antisense oligodeoxynucleotides into intact smooth muscle." Circ Res 77(2): 220-230.

Liu, G., Niu, X., Wu, R. S., Chudasama, N., Yao, Y., Jin, X., Weinberg, R., Zakharov, S. I., Motoike, H., Marx, S. O. and Karlin, A. (2010). "Location of modulatory beta subunits in BK potassium channels." J Gen Physiol 135(5): 449-459.

Liu, J., Asuncion-Chin, M., Liu, P. and Dopico, A. M. (2006). "CaM kinase II phosphorylation of slo Thr107 regulates activity and ethanol responses of BK channels." Nat Neurosci 9(1): 41-49.

Liu, J., Bukiya, A. N., Kuntamallappanavar, G., Singh, A. K. and Dopico, A. M. (2013). "Distinct sensitivity of slol channel proteins to ethanol." Mol Pharmacol 83(1): 235-244.

Liu, J., Vaithianathan, T., Manivannan, K., Parrill, A. and Dopico, A. M. (2008). "Ethanol modulates BKCa channels by acting as an adjuvant of calcium." Mol Pharmacol 74(3): 628-640.

Liu, P., Liu, J., Huang, W., Li, M. D. and Dopico, A. M. (2003). "Distinct regions of the slo subunit determine differential BKCa channel responses to ethanol." Alcohol Clin Exp Res 27(10): 1640-1644.

Liu, P., Xi, Q., Ahmed, A., Jaggar, J. H. and Dopico, A. M. (2004). "Essential role for smooth muscle BK channels in alcohol-induced cerebrovascular constriction." Proc Natl Acad Sci U S A 101(52): 18217-18222. 
Lohn, M., Lauterbach, B., Haller, H., Pongs, O., Luft, F. C. and Gollasch, M. (2001). "beta(1)-Subunit of BK channels regulates arterial wall[Ca(2+)] and diameter in mouse cerebral arteries." J Appl Physiol (1985) 91(3): 1350-1354.

Marmot, M. and Brunner, E. (1991). "Alcohol and cardiovascular disease: the status of the U shaped curve." BMJ 303(6802): 565-568.

Martin, G., Puig, S., Pietrzykowski, A., Zadek, P., Emery, P. and Treistman, S. (2004). "Somatic localization of a specific large-conductance calcium-activated potassium channel subtype controls compartmentalized ethanol sensitivity in the nucleus accumbens." J Neurosci 24(29): 6563-6572.

Meera, P., Wallner, M., Jiang, Z. and Toro, L. (1996). "A calcium switch for the functional coupling between alpha (hslo) and beta subunits (KV,Ca beta) of maxi K channels." FEBS Lett 382(1-2): 84-88.

Meissner, G., Rousseau, E. and Lai, F. A. (1989). "Structural and functional correlation of the trypsin-digested $\mathrm{Ca} 2+$ release channel of skeletal muscle sarcoplasmic reticulum." J Biol Chem 264(3): 1715-1722.

Miledi, R. and Parker, I. (1984). "Chloride current induced by injection of calcium into Xenopus oocytes." J Physiol 357: 173-183.

Mohler, E. R., 3rd, Delanty, N., Rader, D. J. and Raps, E. C. (1999). "Statins and cerebrovascular disease: plaque attack to prevent brain attack." Vasc Med 4(4): 269-272.

Monod, J., Wyman, J. and Changeux, J. P. (1965). "On the Nature of Allosteric Transitions: A Plausible Model." J Mol Biol 12: 88-118.

Moore, R. D. and Pearson, T. A. (1986). "Moderate alcohol consumption and coronary artery disease. A review." Medicine (Baltimore) 65(4): 242-267.

Morrow, J. P., Zakharov, S. I., Liu, G., Yang, L., Sok, A. J. and Marx, S. O. (2006). "Defining the BK channel domains required for beta1-subunit modulation." Proc Natl Acad Sci U S A 103(13): 5096-5101.

Nimigean, C. M. and Magleby, K. L. (2000). "Functional coupling of the beta(1) subunit to the large conductance $\mathrm{Ca}(2+)$-activated $\mathrm{K}(+)$ channel in the absence of $\mathrm{Ca}(2+)$. Increased $\mathrm{Ca}(2+)$ sensitivity from a $\mathrm{Ca}(2+)$-independent mechanism." J Gen Physiol 115(6): 719-736.

Niu, X., Qian, X. and Magleby, K. L. (2004). "Linker-gating ring complex as passive spring and $\mathrm{Ca}(2+)$-dependent machine for a voltage- and $\mathrm{Ca}(2+)$-activated potassium channel." Neuron 42(5): 745-756. 
Orio, P. and Latorre, R. (2005). "Differential effects of beta 1 and beta 2 subunits on BK channel activity." J Gen Physiol 125(4): 395-411.

Orio, P., Rojas, P., Ferreira, G. and Latorre, R. (2002). "New disguises for an old channel: MaxiK channel beta-subunits." News Physiol Sci 17: 156-161.

Patterson, A. J., Henrie-Olson, J. and Brenner, R. (2002). "Vasoregulation at the molecular level: a role for the betal subunit of the calcium-activated potassium (BK) channel." Trends Cardiovasc Med 12(2): 78-82.

Perez, G. J., Bonev, A. D. and Nelson, M. T. (2001). "Micromolar Ca(2+) from sparks activates $\mathrm{Ca}(2+)$-sensitive $\mathrm{K}(+)$ channels in rat cerebral artery smooth muscle." Am J Physiol Cell Physiol 281(6): C1769-1775.

Pluger, S., Faulhaber, J., Furstenau, M., Lohn, M., Waldschutz, R., Gollasch, M., Haller, H., Luft, F. C., Ehmke, H. and Pongs, O. (2000). "Mice with disrupted BK channel beta1 subunit gene feature abnormal $\mathrm{Ca}(2+)$ spark/STOC coupling and elevated blood pressure." Circ Res 87(11): E53-60.

Potter, J. F. and Beevers, D. G. (1984). "Pressor effect of alcohol in hypertension." Lancet 1(8369): 119-122.

Puddey, I. B., Beilin, L. J. and Vandongen, R. (1987). "Regular alcohol use raises blood pressure in treated hypertensive subjects. A randomised controlled trial." Lancet 1(8534): 647-651.

Puddey, I. B., Beilin, L. J., Vandongen, R., Rouse, I. L. and Rogers, P. (1985). "Evidence for a direct effect of alcohol consumption on blood pressure in normotensive men. A randomized controlled trial." Hypertension 7(5): 707-713.

Puddey, I. B., Rakic, V., Dimmitt, S. B. and Beilin, L. J. (1999). "Influence of pattern of drinking on cardiovascular disease and cardiovascular risk factors--a review." Addiction 94(5): 649-663.

Qian, X., Nimigean, C. M., Niu, X., Moss, B. L. and Magleby, K. L. (2002). "Slo1 tail domains, but not the $\mathrm{Ca} 2+$ bowl, are required for the beta 1 subunit to increase the apparent Ca2+ sensitivity of BK channels." J Gen Physiol 120(6): 829-843.

Reynolds, K., Lewis, B., Nolen, J. D., Kinney, G. L., Sathya, B. and He, J. (2003). "Alcohol consumption and risk of stroke: a meta-analysis." JAMA 289(5): 579-588.

Rimm, E. B., Klatsky, A., Grobbee, D. and Stampfer, M. J. (1996). "Review of moderate alcohol consumption and reduced risk of coronary heart disease: is the effect due to beer, wine, or spirits." Bmj 312(7033): 731-736. 
Salkoff, L., Butler, A., Ferreira, G., Santi, C. and Wei, A. (2006). "High-conductance potassium channels of the SLO family." Nat Rev Neurosci 7(12): 921-931.

Santi, C. M., Ferreira, G., Yang, B., Gazula, V. R., Butler, A., Wei, A., Kaczmarek, L. K. and Salkoff, L. (2006). "Opposite regulation of Slick and Slack K+ channels by neuromodulators." J Neurosci 26(19): 5059-5068.

Schreiber, M., Wei, A., Yuan, A., Gaut, J., Saito, M. and Salkoff, L. (1998). "Slo3, a novel $\mathrm{pH}$-sensitive $\mathrm{K}+$ channel from mammalian spermatocytes." J Biol Chem 273(6): 3509-3516.

Seppa, K., Laippala, P. and Sillanaukee, P. (1996). "High diastolic blood pressure: common among women who are heavy drinkers." Alcohol Clin Exp Res 20(1): 47-51.

Shi, J., Krishnamoorthy, G., Yang, Y., Hu, L., Chaturvedi, N., Harilal, D., Qin, J. and Cui, J. (2002). "Mechanism of magnesium activation of calcium-activated potassium channels." Nature 418(6900): 876-880.

Stefani, E., Ottolia, M., Noceti, F., Olcese, R., Wallner, M., Latorre, R. and Toro, L. (1997). "Voltage-controlled gating in a large conductance Ca2+-sensitive $\mathrm{K}+$ channel (hslo)." Proc Natl Acad Sci U S A 94(10): 5427-5431.

Subramani, J., Kathirvel, K., Leo, M. D., Kuntamallappanavar, G., Uttam Singh, T. and Mishra, S. K. (2009). "Atorvastatin restores the impaired vascular endotheliumdependent relaxations mediated by nitric oxide and endothelium-derived hyperpolarizing factors but not hypotension in sepsis." J Cardiovasc Pharmacol 54(6): 526-534.

Sun, X., Shi, J., Delaloye, K., Yang, X., Yang, H., Zhang, G. and Cui, J. (2013). "The interface between membrane-spanning and cytosolic domains in $\mathrm{Ca}(2)+$-dependent $\mathrm{K}+$ channels is involved in beta subunit modulation of gating." J Neurosci 33(27): 1125311261.

Suter, P. M. and Vetter, W. (1999). "Alcohol and ischemic stroke." Nutr Rev 57(10): 310314.

Tanaka, Y., Meera, P., Song, M., Knaus, H. G. and Toro, L. (1997). "Molecular constituents of maxi $\mathrm{KCa}$ channels in human coronary smooth muscle: predominant alpha + beta subunit complexes." J Physiol 502 ( Pt 3): 545-557.

Thomas-Gatewood, C., Neeb, Z. P., Bulley, S., Adebiyi, A., Bannister, J. P., Leo, M. D. and Jaggar, J. H. (2011). "TMEM16A channels generate $\mathrm{Ca}(2)(+)$-activated $\mathrm{Cl}(-)$ currents in cerebral artery smooth muscle cells." Am J Physiol Heart Circ Physiol 301(5): H18191827.

Treistman, S. N. and Martin, G. E. (2009). "BK Channels: mediators and models for alcohol tolerance." Trends Neurosci 32(12): 629-637. 
Uebele, V. N., Lagrutta, A., Wade, T., Figueroa, D. J., Liu, Y., McKenna, E., Austin, C. P., Bennett, P. B. and Swanson, R. (2000). "Cloning and functional expression of two families of beta-subunits of the large conductance calcium-activated $\mathrm{K}+$ channel." J Biol Chem 275(30): 23211-23218.

Wallner, M., Meera, P., Ottolia, M., Kaczorowski, G. J., Latorre, R., Garcia, M. L., Stefani, E. and Toro, L. (1995). "Characterization of and modulation by a beta-subunit of a human maxi KCa channel cloned from myometrium." Receptors Channels 3(3): 185199.

Wallner, M., Meera, P. and Toro, L. (1996). "Determinant for beta-subunit regulation in high-conductance voltage-activated and $\mathrm{Ca}(2+)$-sensitive $\mathrm{K}+$ channels: an additional transmembrane region at the N terminus." Proc Natl Acad Sci U S A 93(25): 1492214927.

Wallner, M., Meera, P. and Toro, L. (1999). "Molecular basis of fast inactivation in voltage and $\mathrm{Ca} 2+$-activated $\mathrm{K}+$ channels: a transmembrane beta-subunit homolog." Proc Natl Acad Sci U S A 96(7): 4137-4142.

Walters, F. S., Covarrubias, M. and Ellingson, J. S. (2000). "Potent inhibition of the aortic smooth muscle maxi-K channel by clinical doses of ethanol." Am J Physiol Cell Physiol 279(4): C1107-1115.

Wannamethee, S. G. and Shaper, A. G. (1996). "Patterns of alcohol intake and risk of stroke in middle-aged British men." Stroke 27(6): 1033-1039.

Wechsler, H., Lee, J. E., Kuo, M., Seibring, M., Nelson, T. F. and Lee, H. (2002). "Trends in college binge drinking during a period of increased prevention efforts. Findings from 4 Harvard School of Public Health College Alcohol Study surveys: 19932001." J Am Coll Health 50(5): 203-217.

Wellman, G. C., Nathan, D. J., Saundry, C. M., Perez, G., Bonev, A. D., Penar, P. L., Tranmer, B. I. and Nelson, M. T. (2002). "Ca2+ sparks and their function in human cerebral arteries." Stroke 33(3): 802-808.

Wellman, G. C. and Nelson, M. T. (2003). "Signaling between SR and plasmalemma in smooth muscle: sparks and the activation of $\mathrm{Ca} 2+$-sensitive ion channels." Cell Calcium 34(3): 211-229.

Welsh, D. G., Morielli, A. D., Nelson, M. T. and Brayden, J. E. (2002). "Transient receptor potential channels regulate myogenic tone of resistance arteries." Circ Res 90(3): 248-250.

Wu, R. S., Chudasama, N., Zakharov, S. I., Doshi, D., Motoike, H., Liu, G., Yao, Y., Niu, X., Deng, S. X., Landry, D. W., Karlin, A. and Marx, S. O. (2009). "Location of the beta 4 transmembrane helices in the BK potassium channel." J Neurosci 29(26): 8321-8328. 
Wu, R. S., Liu, G., Zakharov, S. I., Chudasama, N., Motoike, H., Karlin, A. and Marx, S. O. (2013). "Positions of beta2 and beta 3 subunits in the large-conductance calcium- and voltage-activated BK potassium channel." J Gen Physiol 141(1): 105-117.

Wynne, P. M., Puig, S. I., Martin, G. E. and Treistman, S. N. (2009).

"Compartmentalized beta subunit distribution determines characteristics and ethanol sensitivity of somatic, dendritic, and terminal large-conductance calcium-activated potassium channels in the rat central nervous system." J Pharmacol Exp Ther 329(3): 978-986.

Xi, Q., Adebiyi, A., Zhao, G., Chapman, K. E., Waters, C. M., Hassid, A. and Jaggar, J. H. (2008). "IP3 constricts cerebral arteries via IP3 receptor-mediated TRPC3 channel activation and independently of sarcoplasmic reticulum $\mathrm{Ca} 2+$ release." Circ Res 102(9): 1118-1126.

Xia, X. M., Ding, J. P. and Lingle, C. J. (1999). "Molecular basis for the inactivation of $\mathrm{Ca} 2+-$ and voltage-dependent BK channels in adrenal chromaffin cells and rat insulinoma tumor cells." J Neurosci 19(13): 5255-5264.

Xia, X. M., Ding, J. P. and Lingle, C. J. (2003). "Inactivation of BK channels by the NH2 terminus of the beta 2 auxiliary subunit: an essential role of a terminal peptide segment of three hydrophobic residues." J Gen Physiol 121(2): 125-148.

Xia, X. M., Zeng, X. and Lingle, C. J. (2002). "Multiple regulatory sites in largeconductance calcium-activated potassium channels." Nature 418(6900): 880-884.

Xia, X. M., Zhang, X. and Lingle, C. J. (2004). "Ligand-dependent activation of Slo family channels is defined by interchangeable cytosolic domains." J Neurosci 24(24): 5585-5591.

Xu, L., Jones, R. and Meissner, G. (1993). "Effects of local anesthetics on single channel behavior of skeletal muscle calcium release channel." J Gen Physiol 101(2): 207-233.

Yang, Z., Wang, J., Zheng, T., Altura, B. T. and Altura, B. M. (2001). "Importance of extracellular $\mathrm{Ca} 2+$ and intracellular $\mathrm{Ca} 2+$ release in ethanol-induced contraction of cerebral arterial smooth muscle." Alcohol 24(3): 145-153.

Ye, S., Li, Y., Chen, L. and Jiang, Y. (2006). "Crystal structures of a ligand-free MthK gating ring: insights into the ligand gating mechanism of $\mathrm{K}+$ channels." Cell 126(6): 1161-1173.

Yuan, C., Chen, M., Covey, D. F., Johnston, L. J. and Treistman, S. N. (2011). "Cholesterol tuning of BK ethanol response is enantioselective, and is a function of accompanying lipids." PLoS One 6(11): e27572. 
Yuan, P., Leonetti, M. D., Hsiung, Y. and MacKinnon, R. (2012). "Open structure of the $\mathrm{Ca} 2+$ gating ring in the high-conductance $\mathrm{Ca} 2+$-activated $\mathrm{K}+$ channel." Nature 481(7379): 94-97.

Yusifov, T., Savalli, N., Gandhi, C. S., Ottolia, M. and Olcese, R. (2008). "The RCK2 domain of the human BKCa channel is a calcium sensor." Proc Natl Acad Sci US A 105(1): 376-381.

Zakhari, S. (1997). "Alcohol and the cardiovascular system: molecular mechanisms for beneficial and harmful action." Alcohol Health Res World 21(1): 21-29.

Zhang, A., Altura, B. T. and Altura, B. M. (1993). "Ethanol-induced contraction of cerebral arteries in diverse mammals and its mechanism of action." Eur J Pharmacol 248(3): 229-236.

Zhang, Z., Rosenhouse-Dantsker, A., Tang, Q. Y., Noskov, S. and Logothetis, D. E. (2010). "The RCK2 domain uses a coordination site present in Kir channels to confer sodium sensitivity to Slo2.2 channels." J Neurosci 30(22): 7554-7562. 


\section{VITA}

Guruprasad Kuntamallappanavar was born on March 22, 1984 in Karnataka, India. He graduated from high school in 2000. He pursued his Bachelor of Veterinary Science (B.V.Sc) degree from Veterinary College, Karnataka Veterinary Animal Fisheries Sciences University, Bidar, India in August, 2007. Later, he obtained his Masters of Veterinary Sciences (M.V.Sc.) degree from Indian Veterinary Research Institute, Uttar Pradesh, India in August, 2009. In August 2009, he joined the Integrated Program in Biomedical Sciences (IPBS) at the University of Tennessee Health Science Center (UTHSC), Memphis, Tennessee. Currently, he is a member of the Biophysical Society and the Research Society on Alcoholism. He is a recipient of the most prestigious predoctoral fellowship award (January 2013 - December 2014) from the American Heart Association, Greater Southeast Affiliate. He was also a recipient of four travel awards during his graduate studies: a Biophysical Society Education Travel award (2013) a Research Society on Alcoholism Student Merit Award (2014), and two educational travel awards from the College of Graduate Health Sciences, UTHSC in 2013 and 2014. These awards helped him to present his scientific work at national and international scientific meetings, such as the Biophysical Society, Research Society on Alcoholism and International Society for Biomedical Research on Alcoholism meetings. He was awarded his Ph.D. from the University of Tennessee Health Science Center, Memphis, Tennessee, in May, 2015. 